\title{
Real-time intracellular temperature imaging using lanthanide-bearing polymeric micelles
}

Rafael Piñol ${ }^{1}$, Justyna Zeler ${ }^{2,3}$, Carlos D. S. Brites ${ }^{2}$, Yuanyu $\mathrm{Gu}^{1,4}$, Pedro Téllez ${ }^{5}$, Albano N. Carneiro Neto ${ }^{2}$, Thiago E. da Silva ${ }^{2,6}$, Raquel Moreno-Loshuertos ${ }^{7}$, Patrício Fernandez-Silva7, Ana Gallego ${ }^{7}$, Luis Martinez-Lostao7, Abelardo Martínez ${ }^{8}$, Luís D. Carlos $^{2^{*}}$, Angel Millán ${ }^{1^{*}}$

${ }^{1}$ ICMA, Institute of Materials Science of Aragon, CSIC-University of Zaragoza, 50008 Zaragoza, Spain

2 Phantom-g, CICECO-Aveiro Institute of Materials, Department of Physics, University of Aveiro, Campus de Santiago, 3810-193 Aveiro, Portugal

${ }^{3}$ Faculty of Chemistry, University of Wroclaw, Wroclaw, Poland

${ }^{4}$ School of Materials Science and Engineering. Nanjing Tech University, 210009, Nanjing PR China

${ }^{5}$ Servicio de Apoyo a la Investigación. University of Zaragoza, C/ Pedro Cerbuna 10, 50006 Zaragoza, Spain

${ }^{6}$ Department of Fundamental Chemistry, Federal University of Pernambuco, 50670901, Recife, PE, Brazil.

${ }^{7}$ Departamento de Bioquímica, Biología Molecular y Celular, Universidad de Zaragoza, Zaragoza, Spain

8 Departamento de Electrónica de Potencia, I3A, Universidad de Zaragoza 50018 Zaragoza, Spain

Measurement of thermogenesis in individual cells is a remarkable challenge due to the complexity of the biochemical environment (such as $\mathrm{pH}$ and ionic strength) and to the rapid and yet not well-understood heat transfer mechanisms throughout the cell. Here, we present a unique system for intracellular temperature mapping in a fluorescence microscope (uncertainty of $0.2 \mathrm{~K}$ ) using rationally designed luminescent $\mathrm{Ln}^{3+}$-bearing polymeric micellar probes ( $\mathrm{Ln}=\mathrm{Sm}$, Eu) incubated in breast cancer MDA-MB468 cells.

2D thermal images recorded increasing the temperature of the cells culture medium between 296 and $304 \mathrm{~K}$ shows inhomogeneous intracellular temperature progressions up to $\sim 20$ degrees and subcellular gradients of $\sim 5$ degrees between the nucleolus and the rest of the cell, illustrating the thermogenic activity of the different organelles and highlighting the potential of this tool to study intracellular processes.

Keywords: Intracellular temperature mapping, Luminescence thermometry, Thermogenesis, Lanthanide-based polymer nanomicelles 
Temperature affects everything and temperature fluctuations play a central role in the myriad of biochemical reactions regulating life. ${ }^{1}$ The intracellular temperature, for instance, changes with cellular activity, including cell division, gene expression, enzymatic reactions, and pathological states..$^{2-4}$ Moreover, to neutralize large external temperature changes and to keep homeostasis of the body temperature upon cold or hot exposure, cells have developed thermoregulation mechanisms. ${ }^{3-7}$ Despite recognition of the fundamental involvement of temperature in eliciting biochemical changes, ${ }^{8-10}$ specific molecular mechanisms by which cells produce and use heat are largely unknown,, , 7, 11-18, which, thus, have driven the research on intracellular thermometers in the past decade. Although there are a handful of reported intrusive intracellular thermometers, ${ }^{6,19,20}$ contactless luminescence-based examples have been largely the most adopted strategy. ${ }^{9}$ 10, 21-27 Indeed, several types of luminescent materials have been proposed for intracellular temperature sensing such as organic molecules, ${ }^{21}, \quad 28, \quad 29$ polymers, $^{30}, \quad 31$ carbon dots, ${ }^{32}$ quantum dots, ${ }^{33} \mathrm{~N}$-vacancy nanodiamonds, ${ }^{2}$ and trivalent lanthanide ions $\left(\mathrm{Ln}^{3+}\right)$-doped nanomaterials. ${ }^{34}$

These luminescent thermometers unveiled intriguing spatiotemporal intracellular temperature fluctuations, including organelle-specific thermogenesis, suggesting a fundamental connection between the temperature and the cell functions. ${ }^{4,}$ 7-10 For example, Chrétien et al. ${ }^{4,7-10}$ correlated changes in the mitochondrial temperature with the oxygen consumption, showing that the mitochondrial temperature is up to 10 degrees higher than the body temperature when the respiratory chain was fully functional. Also, Tanimoto et al. ${ }^{35}$ observed temperature differences up to 6 degrees in micrometer scale domains in a neuronal cell after the mitochondrial stimulation, whereas Rajagopal et al. ${ }^{6}$ reported temperature spikes of $\sim 7.5$ degrees during stimulated proton uncoupling in neurons of Aplysia californica. Finally, Yang et al. ${ }^{33}$ and Liu et al. ${ }^{36}$ showed inhomogeneous intracellular temperature distributions up to $10-15$ degrees higher than the temperature of the living cells culture medium, following, respectively, $\mathrm{Ca}^{2+}$ stress and cold shock, and externally-induced temperature changes. 
All these observations (among other examples ${ }^{5,30}$ ) are inconsistent with theoretical calculations suggesting that maximal heat gradients across cells could not be greater than $10^{-5} \mathrm{~K}^{5,30}$

Here, we report intriguing real-time bidimensional (2D) temperature maps of breast metastatic adenocarcinoma cells (line MDA-MB-468) incubated with innovative $\mathrm{Ln}^{3+}$ bearing polymeric micelles. The thermal images show inhomogeneous intracellular temperature progressions up to $\sim 20$ degrees and subcellular temperature gradients of $\sim 5$ degrees raising the exciting possibility of using these gradients for signaling and regulation in cells, ${ }^{33}$ and emphasizing the present misunderstood about the response of living cells to temperature variations.

A scheme of the synthesis of the micelles is presented in Figure 1. It consists of a self-organized structure formed using a single polymer containing hydrophilic and hydrophobic blocks. The amphiphilic block is the P(MPEGA-co-PEGA)-b-P(MA-bPhenA) copolymer (MPEGA and PEGA stand for poly(ethylene glycol) methyl ether acrylate and polyethyleneglycol acrylate, respectively) and was synthesized by reversible addition-fragmentation chain transfer polymerization (details in the Supporting Information). The hydrophobic block is a methyl acrylate (MA) polymer containing a discrete number of the auxiliary ligand 4-(acryloyloxymethyl)-1,10phenanthroline (PhenA) to complete the coordination sphere of the $\mathrm{Ln}^{3+}$ ion and shield the complex from the influence of the medium by steric effects. We rationally design the polymeric micelles and the $\mathrm{Ln}^{3+}$ complexes to mitigate luminescence quenching effects induced by the culture medium and the proposed copolymerization strategy permits easy incorporation of other functionalities such as tags for different cell organelles. The hydrodynamic diameter of the resulting micelles in aqueous suspension gives a monomodal distribution with an average diameter of $20.3 \pm 0.2 \mathrm{~nm}$ (Figure 1b,c). The scanning transmission electron micrographs showed particles, with a granular texture and bright spots (discernible at high magnification), which is compatible with the presence of $\mathrm{Ln}^{3+}$ complexes within the micelle. Moreover, the 
electron dispersive scattering (EDS) within the micelles presents the signature of Sm, whereas less frequent $\mathrm{Eu}$, was only faintly detected (Figure S2 in Supporting Information). The experimental ratios of the $\mathrm{Ln}^{3+}$ ions were $2.42 \%$ and $2.84 \% \mathrm{w} / \mathrm{w}$, for DNDP and btfa complexes, respectively. These elements were not observed outside the micelles indicating that there is no leakage of the $\mathrm{Ln}^{3+}$ ions to the suspension (Figures S3-S5 in Supporting Information).

The photoluminescence studies on the $\mathrm{Ln}^{3+}$-bearing polymeric micelles are summarized in Figure 2. A remarkable excitation peak at $436 \mathrm{~nm}$, in the blue-violet spectral range, is discerned (Figure 2a). This is an important achievement because contrary to other down-shifting $\mathrm{Ln}^{3+}$-based thermometers that need high-energetic (and cell harmful) ultraviolet excitation, ${ }^{37}$ the excitation in the visible spectral range circumvents damage to the cultured cells under observation and it can be easily used in conventional confocal microscopes and cytometers that do not natively incorporate UV laser sources. Moreover, as these instruments do not also generally incorporate NIR laser sources, intracellular thermometers based on $\mathrm{Ln}^{3+}$-doped upconverting nanoparticles ${ }^{34}$ and nanocomposites ${ }^{38-40}$ (generally excited at $980 \mathrm{~nm}$ ) cannot be used routinely, despite the negligible autofluorescence of the cells induced by NIR excitation.

The thermal dependence of the emission spectra was recorded in the $300-328 \mathrm{~K}$ range for the aqueous suspension of the $\mathrm{Eu}^{3+}$ - and $\mathrm{Sm}^{3+}$-bearing polymeric micelles (Figure $2 \mathrm{~b}-\mathrm{d}$ ). In the case of btfa-based micelles, the integrated area of the $\mathrm{Sm}^{3+}$ ${ }^{4} \mathrm{G}_{5 / 2} \rightarrow{ }^{6} \mathrm{H}_{9 / 2}$ transition $\left(I_{1}\right)$ decreases with increasing temperature by $\sim 12 \%$, that of the $\mathrm{Eu}^{3+},{ }^{5} \mathrm{D}_{0} \rightarrow{ }^{7} \mathrm{~F}_{2}$ transition $\left(I_{2}\right)$ is reduced by $40 \%$ of the initial value (Figure $3 \mathrm{a}$ ). According to the DNPD-based material, the temperature-dependent trend is more evident. The emission of $\mathrm{Eu}^{3+}$ decreases increasing temperature (leaving $\sim 18 \%$ of the $\mathrm{RT}$ intensity), and what is more also the emission of $\mathrm{Sm}^{3+}$ is reduced $\sim 4$ times at the highest temperature (Figure 3b). To rationalize these thermal dependences, we use time-dependent density functional theory and intramolecular energy transfer 
calculations (details in Supporting Information). The intramolecular energy transfer rates for $\mathrm{Ln}^{3+}$ complexes were estimated based on a well-defined theory. ${ }^{41-45}$

The main energy-transfer pathways in the micelles containing the DNPD complexes (Figure 3c) involves the $\mathrm{T}_{1} \rightarrow{ }^{5} \mathrm{D}_{0}\left(\mathrm{Eu}^{3+}\right)$ and $\mathrm{T}_{1} \rightarrow{ }^{4} \mathrm{G}_{5 / 2}\left(\mathrm{Sm}^{3+}\right)$ transitions corresponding respectively to $\sim 83 \%$ and $\sim 91 \%$ of the total forward intramolecular energy transfer rates. On one hand, the backward energy transfer rates $\left(W_{\mathrm{bET}}\right)$ increase at temperature increase, depopulating the ${ }^{5} \mathrm{D}_{0}\left(\mathrm{Eu}^{3+}\right)$ and ${ }^{4} \mathrm{G}_{5 / 2}\left(\mathrm{Sm}^{3+}\right)$ emitting levels and, on the other hand, $W_{\mathrm{bET}}$ for $\mathrm{Eu}^{3+}$ is more affected by the temperature increase than those of $\mathrm{Sm}^{3+}$. The balance between these two processes explains the stepper decay observed for the integrated area of ${ }^{5} D_{0} \rightarrow{ }^{7} F_{2}$ transition in comparison with that of ${ }^{4} \mathrm{G}_{5 / 2} \rightarrow{ }^{6} \mathrm{H}_{9 / 2}$ (Figure 3b). In the btfa-Ln ${ }^{3+}$ complexes, the $\mathrm{T}_{1}$ state (15480 $\mathrm{cm}^{-1}$ ) is lying below the $\mathrm{Eu}^{3+}$ and $\mathrm{Sm}^{3+}$ ion acceptor states, leading to a higher energy barrier. Consequently, the energy transfer pathways involving the $S_{1}$ state $\left(34300 \mathrm{~cm}^{-1}\right)$ as donor become more operative (in order of $10^{4}$ and $10^{6} \mathrm{~s}^{-1}$, respectively, Tables S5 and S6). According to the simulations, the energy transfer from the $\mathrm{S}_{1}$ represents $93.4 \%$ and $93.8 \%$ of the total for $\mathrm{Eu}^{3+}$ and $\mathrm{Sm}^{3+}$ ions, respectively. For the btfa ligand, the energy transfer involves the non-radiative decays from the lanthanides' higher energy states to the corresponding emitting levels. Since the population of the emitting levels is dependent on its decay lifetimes (that are in order of $10^{-3} \mathrm{~s}$ ), it has time enough to transfer energy to the triplet state (in order of $10^{4} \mathrm{~s}^{-1}$ for $\mathrm{Eu}^{3+}$ and $10^{5} \mathrm{~s}^{-1}$ for $\mathrm{Sm}^{3+}$ ). This means that the population of the emitting level is dependent on the balance between the population increase resulting from the singlet $\left(\mathrm{W}_{\mathrm{Ln}}^{\mathrm{S}}\right)$ and the population decrease due to lanthanide-to-btfa triplet state $\left(\mathrm{W}_{\mathrm{T}_{1}}^{\mathrm{Ln}}\right)$ energy transfers, which allow us to define an effective transition rate as $\mathrm{W}^{*}=\mathrm{W}_{\mathrm{Ln}}^{\mathrm{S}}-\mathrm{W}_{\mathrm{T}_{1}}^{\mathrm{Ln}}$. Figure $\mathrm{S} 7$ presents the temperature dependence of the normalized $\mathrm{W}^{*}$. The rate for the $\mathrm{Eu}^{3+} \rightarrow \mathrm{T}_{1}$ energy transfer is of the same order of magnitude of that of $\mathrm{S}_{1} \rightarrow \mathrm{Eu}^{3+}$ in the higher energetic states. In contrast, for the btfa-Sm ${ }^{3+}$ complex, the $\mathrm{W}_{\mathrm{T}_{1}}^{\mathrm{Ln}}$ is one order of magnitude lower 
than the $\mathrm{W}_{\mathrm{Ln}}^{\mathrm{S}}$, explaining the lower temperature quenching of the $\mathrm{Sm}^{3+}$ integrated area in comparison with the $\mathrm{Eu}^{3+}$ one presented in Figure 3a.

The integrated areas of the ${ }^{4} \mathrm{G}_{5 / 2} \rightarrow{ }^{6} \mathrm{H}_{9 / 2}\left(I_{1}, \mathrm{Sm}^{3+}\right)$ and ${ }^{5} \mathrm{D}_{0} \rightarrow{ }^{7} \mathrm{~F}_{2}\left(I_{2}, \mathrm{Eu}^{3+}\right)$ transitions are used to define the thermometric parameter $\Delta=I_{1} / I_{2}$. The $\Delta(T)$ dependence is well fitted by empirical polynomial equations for both ligands (Figure S13 in Supporting Information). The relative thermal sensitivity (Figure S14 in Supporting Information) of the micelles increases with the temperature increase reaching the maximum value of $1.5 \% . \mathrm{K}^{-1}$ and $1.7 \% . \mathrm{K}^{-1}$ for btfa- and DNPD-containing micelles, respectively, both at $328 \mathrm{~K}$. The minimum temperature uncertainty is remarkably low, $6 \mathrm{mK}$ (at $328 \mathrm{~K}$ ), and $0.20 \mathrm{~K}($ at $305 \mathrm{~K}$ ) for the btfa- and DNPD-containing micelles (Figure S16 in Supporting Information). Whereas the relative thermal sensitivity values are lower than that reported for similar micelles containing $\mathrm{Tb}^{3+}$ ions ${ }^{37}\left(5.8 \% \cdot \mathrm{K}^{-1}\right.$ at $\left.296 \mathrm{~K}\right)$, the decrease of the relative uncertainty on the integrated intensity to unprecedented values of $\delta \mathrm{I} / \mathrm{l}<0.4 \%$ (Figure $\mathrm{S} 15$ in Supporting Information) allowed us to design the first luminescent thermometer with temperature uncertainty values below $10 \mathrm{mK}$.

These exciting results of thermometric performance motivate the development of a temperature-imaging instrument (Figure S7 in Supporting Information). It consists of a fluorescence microscope that incorporates a beam splitter, a CMOS camera, and a CCD camera. The illumination light is filtered through a $340-380 \mathrm{~nm}$ bandpass filter and reflected with a $400 \mathrm{~nm}$ dichroic mirror. The emission beam is divided into two by a $635 \mathrm{~nm}$ dichroic mirror and the resulting beams are filtered through the 600-620 nm and $620-660 \mathrm{~nm}$ bandpass filters that roughly cover the ${ }^{5} \mathrm{D}_{0} \rightarrow{ }^{7} \mathrm{~F}_{2}\left(\mathrm{Eu}^{3+}\right)$ and ${ }^{4} \mathrm{G}_{5 / 2} \rightarrow{ }^{6} \mathrm{H}_{9 / 2}\left(\mathrm{Sm}^{3+}\right)$ emissions. Finally, both the $\mathrm{Eu}^{3+}$ and $\mathrm{Sm}^{3+}$ filtered beams are collected by a" CMOS camera (Figure S7b in Supporting Information). All the process of temperature imaging is controlled by a LabView image-processing sequence. A MatLab® routine (developed by us) is then used to process the original CMOS image containing both the $\mathrm{Eu}^{3+}$ and $\mathrm{Sm}^{3+}$ emission to get $2 \mathrm{D}$ temperature maps (Figure $\mathrm{S} 7 \mathrm{~b}$ in Supporting Information). The temperature-mapping routine first separates the original 
image into the corresponding $\mathrm{Sm}^{3+}\left(I_{1}\right)$ and $\mathrm{Eu}^{3+}\left(I_{2}\right)$ emissions. Then, the intensity values for the equivalent pixels of $\mathrm{Eu}^{3+}$ and $\mathrm{Sm}^{3+}$ images are identified, and the intensity ratio $\Delta=I_{1} / I_{2}$ is calculated and converted into temperature by the corresponding calibration curve. Finally, we obtain a 2D temperature matrix that is represented as a false-color image that can be overlapped to the original image, mapping the temperature distribution of the cellular culture.

Despite both the btfa- and DNPD-based $\mathrm{Ln}^{3+}$-bearing polymeric micelles are stable in water, in the presence of the culture medium the btfa-based polymeric micelles present a poor emission intensity in the same excitation conditions of DNPD-based ones (Figure S22 in Supporting Information). The time-dependent density functional theory and the intramolecular energy transfer calculations point out that the btfa-based micelles undergo a luminescence quenching process due to the energy transfer that involves the $\mathrm{Ln}^{3+}$ emitting levels to the $\mathrm{T}_{1}$ state. On the contrary, in the DNPD-based micelles is the balance between the $\mathrm{S}_{1}$-to- $\mathrm{Ln}^{3+}$ and the $\mathrm{Ln}^{3+}$-to- $\mathrm{T}_{1}$ energy transfers that rules the temperature dependence of the intensities (details in silico experiments in Supporting Information).

In a proof-of-concept experiment, the thermometric imaging system was tested with MDA-MB-468 breast cancer cells incubated with DNPD-based polymeric micelles. The cells were observed before and after incubation with the $\mathrm{Ln}^{3+}$-bearing polymeric micelles (culture and cell internalization details in Supporting Information). Phasecontrast images (Figure 4a,b) showed that the cells were well-formed and uniformly spread. Grayscale images showed the luminescence of internalized $\mathrm{Ln}^{3+}$-bearing polymeric micelles in both the $\mathrm{Sm}^{3+}$ and the $\mathrm{Eu}^{3+}$ channels (Figure 4c,d, and Figure $\mathrm{S} 23 \mathrm{c}, \mathrm{d}$ in Supporting Information), whereas images from control cells before incubation with the micelles were totally dark (Figure S18 in Supporting Information). These results are compatible with the cell internalization of the $\mathrm{Ln}^{3+}$-bearing polymeric micelles and indicate their stability on cellular medium. Moreover, the mean temperature determined in both the fixed and live cells agrees very well with the 
temperature values of the cellular culture $(298.0$ and $296.5 \mathrm{~K}$, respectively, Figures S19, S23 and S24 in Supporting Information). Interestingly, the temperature distribution is much narrower in the fixed cells (standard error of $0.02 \mathrm{~K}$, Figures S19 and S23 in Supporting Information) than in the live cell (standard error $>0.5 \mathrm{~K}$ Figure $4 \mathrm{c}, \mathrm{d}$ and Figure S24 in Supporting Information).

The phase-contrast images of the cell cultures incubated with DNPD-based polymeric micelles display brighter regions under UV-light illumination (yellow arrows in Figures $4 \mathrm{c}, \mathrm{d}$ ) corresponding to areas of higher accumulation of the luminescent probes. The intensity-to-temperature calibration curves of these regions recorded both in a suspension of DNPD-based polymeric micelles and in fixed MDA-MB-468 cells incubated with these micelles are analogous (Figure S19 in Supporting Information) demonstrating that the accumulation of the micelles does not impact the temperature dependence of the $\mathrm{Eu}^{3+}$ and $\mathrm{Sm}^{3+}$ emissions. Although the detailed analysis of the distribution of the nanoparticles within the cell lies out of the scope of the present work, and the identification of the spherical brighter areas would require the use of biological markers, from a morphological point of view these spots might correspond to the cells' nucleolus. To evaluate the effect of an external heat source in the temperature distribution at subcellular regions, we use a temperature controller attached to the imaging instrument described above. A total of 450 points were sampled (150 in the brighter and 300 in the darker regions) within the limits of 3 MDA-MB-468 cells for each temperature set between 296.45 and $303.55 \mathrm{~K}$ (Figure 5a-d). The histograms of both dark and bright regions display an inhomogeneous intracellular temperature progression up to $\sim 20$ degrees (Figure 5e-h and Figure S24 in Supporting Information), which is consistent with the previous reports on temperature mappings on living cells using diverse fluorophores as temperature probes $^{17,33,35,36}$ (Table S8). Furthermore, all the histograms follow Gaussian distributions centered at values that increase with the external temperature set in the controller (Figure 5e-i). Remarkably, whereas for the darker regions the average temperatures are in excellent agreement with those set 
externally, for the brighter areas we observe an average temperature $\sim 5$ degrees higher than that of the rest of the cell (Figure 5i). The results in the suspensions of DNPD-based polymeric micelles and fixed MDA-MB-468 cells incubated with these micelles mentioned above dismiss the possibility of this temperature gradient be induced by the clustering of the micelles. Moreover, the observed temperature increment value is of the same order of magnitude than that already observed by Okabe et al. ${ }^{30}$ and could be explained by the intense RNA transcription activity of the nucleolus. ${ }^{46,47}$ The real-time intracellular temperature imaging using these $\mathrm{Ln}^{3+}$-bearing polymeric micelles was recorded using the same procedure (Figure S21 in Supporting Information) to highlight the possibility of using the strategy described above for tracking thermal events occurring in subcellular level.

In summary, we reported here a unique system for real-time intracellular temperature mapping of living cells based on innovative luminescent $\mathrm{Eu}^{3+} / \mathrm{Sm}^{3+}{ }_{-}$ bearing polymeric micellar probes. The micelles and the $\mathrm{Ln}^{3+}$ complexes covalently bonded to its hydrophobic part were rationally designed to mitigate quenching effects induced by the culture medium. Moreover, the copolymerization strategy proposed permits easy incorporation of other functionalities, such as targeting different cell organelles, and the temperature is recorded from the ratio between the integrated areas of the ${ }^{4} \mathrm{G}_{5 / 2} \rightarrow{ }^{6} \mathrm{H}_{9 / 2}$ and ${ }^{5} \mathrm{D}_{0} \rightarrow{ }^{7} \mathrm{~F}_{2}$ transitions of the $\mathrm{Sm}^{3+}$ and $\mathrm{Eu}^{3+}$ ions, respectively, using a conventional (or minimally adapted) fluorescence microscope. A proof-of-concept experiment on breast metastatic adenocarcinoma cells (line MDA-MB468) incubated with the $\mathrm{Eu}^{3+} / \mathrm{Sm}^{3+}$-bearing micelles under UV-light excitation yields $2 \mathrm{D}$ thermal images in the $296-304 \mathrm{~K}$ range with an uncertainty of $0.2 \mathrm{~K}$. The thermal images allow us to delimitate the cells and to identify its nucleolus as brighter regions. By increasing the temperature of the culture medium, inhomogeneous intracellular temperature progressions up to $\sim 20$ degrees and an increment of $\sim 5$ degrees of the nucleolus temperature relative to the rest of the cell were discerned, which might be related to the activity of the thermo-generating organelles and highly exoenergetic 
processes. The real-time intracellular temperature imaging using these $\mathrm{Ln}^{3+}$-bearing polymeric micelles opens new avenues to the detailed screening of thermal gradients within living cells due to endogenous or exogenous stimuli, providing important insights into the regulatory intracellular mechanisms, and thus contributing to an improved comprehension of the role of some subcellular thermo-generating organelles in the cellular functions.

\section{Associated Content}

Supporting Information: Materials and methods, Thermometric-imaging system, In silico experiments, Photoluminescence, Thermometric performance of the $\mathrm{Ln}^{3+}$-bearing micelles, Cell cultures, and Temperature imaging.

\section{Author Information}

\section{Corresponding Authors}

Luís D. Carlos

e-mail: Icarlos@ua.pt

Angel Millán

e-mail: amillan@unizar.es

\section{Author Contributions}

The manuscript was written through the contributions of all authors. RP synthetized the thermometric micelles, JZ recorded the photoluminescent measurements; CDSB wrote the MatLab routines, analyzed the microscopy images and the photoluminescent results, and draw the figures; YG performed the microscopy observations, ANCN and TES performed the in-silico experiments; PT and AM developed the cell temperature imaging experimental set-up; RM, PFS, AG, and LML performed the cell culture experiments; LDC and AM wrote the paper and co-supervised the project. All authors have approved the final version of the manuscript.

\section{Acknowledgments}

This work was developed within the scope of the project CICECO-Aveiro Institute of Materials, UIDB/50011/2020 \& UIDP/50011/2020, financed by Portuguese funds through the FCT/MEC and when appropriate co-financed by FEDER under the PT2020 Partnership Agreement. Financial support from the project (NanoHeatControl, POCl01-0145-FEDER-031469), funded by FEDER, through POCl and by Portuguese funds (OE), through FCT/MCTES, by European Union's Horizon 2020 FET Open program under grant agreements no. 801305 and 829162, Spanish Ministry of Science Innovation and Universities (Grant No: PGC2018_095795_B_100) and Diputación General de Aragón (E11/17R) are acknowledged. ANCN thanks SusPhotoSolutions project, CENTRO-01-0145-FEDER-000005, Portugal for his grant. The authors acknowledge the use of Servicio General de Apoyo a la Investigación-SAI, Universidad de Zaragoza. 


\section{Abbreviations}

btfa - 4,4,4-trifluoro-1-phenyl-1,3-butanedione; DNPD - 1,3-di(naphthalen-2-yl)propane1,3-dione; MPEGA - methoxy polyethylene glycol acrylate; PEGA - polyethylene glycol acrylate; MA - methyl acrylate; PhenA - 1,10-phenanthrolin-4-yl)methyl acrylate. 


\section{References}

1. Precht, H. Temperature and Life; Springer Science \& Business Media: Berlin, 2013.

2. Kucsko, G.; Maurer, P. C.; Yao, N. Y.; Kubo, M.; Noh, H. J.; Lo, P. K.; Park, H.; Lukin, M. D. Nanometre-Scale Thermometry in a Living Cell. Nature 2013, 500, 54-58, DOI: 10.1038/Nature12373.

3. Ibraimov, A. I. Cell Thermoregulation: Problems, Advances and Perspectives. J. Mol. Biol. Res. 2017, 7, 58-79, DOI: 10.5539/jmbr.v7n1p58.

4. Okabe, K.; Sakaguchi, R.; Shi, B.; Kiyonaka, S. Intracellular Thermometry with Fluorescent Sensors for Thermal Biology. Pflug. Arch. Eur. J. Phy. 2018, 470, 717-731, DOI: 10.1007/s00424-018-2113-4.

5. Kiyonaka, S.; Kajimoto, T.; Sakaguchi, R.; Shinmi, D.; Omatsu-Kanbe, M.; Matsuura, H.; Imamura, H.; Yoshizaki, T.; Hamachi, I.; Morii, T., et al. Genetically Encoded Fluorescent Thermosensors Visualize Subcellular Thermoregulation in Living Cells. Nat. Methods 2013, 10, 1232-1238, DOI: 10.1038/Nmeth.2690.

6. Rajagopal, M. C.; Brown, J. W.; Gelda, D.; Valavala, K. V.; Wang, H.; Llano, D. A.; Gillette, R.; Sinha, S. Transient Heat Release During Induced Mitochondrial Proton Uncoupling. Commun. Biol. 2019, 2, 279, DOI: 10.1038/s42003-019-0535-y.

7. Suzuki, M.; Plakhotnik, T. The Challenge of Intracellular Temperature. Biophys. Rev. 2020, 12, 593-600, 10.1007/s12551-020-00683-8.

8. Takei, Y.; Arai, S.; Murata, A.; Takabayashi, M.; Oyama, K.; Ishiwata, S.; Takeoka, S.; Suzuki, M. A Nanoparticle-Based Ratiometric and Self-Calibrated Fluorescent Thermometer for Single Living Cells. ACS Nano 2014, 8, 198-206, DOI: 10.1021/Nn405456e.

9. Bai, T. T.; Gu, N. Micro/Nanoscale Thermometry for Cellular Thermal Sensing. Small 2016, 12, 4590-4610, DOI: 10.1002/smll.201600665.

10. Uchiyama, S.; Gota, C.; Tsuji, T.; Inada, N. Intracellular Temperature Measurements with Fluorescent Polymeric Thermometers. Chem. Commun. 2017, 53, 10976-10992, DOI: 10.1039/c7cc06203f.

11. Baffou, G.; Rigneault, H.; Marguet, D.; Jullien, L. A Critique of Methods for Temperature Imaging in Single Cells. Nat. Methods 2014, 11, 899-901, DOI: 10.1038/nmeth.3073.

12. Arai, S.; Lee, S.-C.; Zhai, D.; Suzuki, M.; Chang, Y. T. A Molecular Fluorescent Probe for Targeted Visualization of Temperature at the Endoplasmic Reticulum. Sci. Rep.-UK 2014, 4, 6701, DOI: 10.1038/srep06701.

13. Kiyonaka, S.; Sakaguchi, R.; Hamachi, I.; Morii, T.; Yoshizaki, T.; Mori, Y. Validating Subcellular Thermal Changes Revealed by Fluorescent Thermosensors. Nat. Methods 2015, 12, 801-802, DOI: 10.1038/nmeth.3548.

14. Baffou, G.; Rigneault, H.; Marguet, D.; Jullien, L. Validating Subcellular Thermal Changes Revealed by Fluorescent Thermosensors and the $10^{5}$ Gap Issue between Calculation and Measurement in Single-Cell Thermometry. Reply. Nat Methods 2015, 12, 803-803, DOI :10.1038/nmeth.3552.

15. Suzuki, M.; Zeeb, V.; Arai, S.; Oyama, K.; Ishiwata, S. The $10^{5}$ Gap Issue between Calculation and Measurement in Single-Cell Thermometry. Nat. Methods 2015, 12, 802-803, DOI: 10.1038/nmeth.3551.

16. Kriszt, R.; Arai, S.; Itoh, H.; Lee, M. H.; Goralczyk, A. G.; Ang, X. M.; Cypess, A. M.; White, A. P.; Shamsi, F.; Xue, R. D., et al. Optical Visualisation of Thermogenesis in Stimulated Single-Cell Brown Adipocytes. Sci. Rep.-UK 2017, 7, 1383, DOI: 10.1038/s41598-017-00291-9.

17. Chrétien, D.; Bénit, P.; Ha, H. H.; Keipert, S.; El-Khoury, R.; Chang, Y. T.; Jastroch, M.; Jacobs, H. T.; Rustin, P.; Rak, M. Mitochondria Are Physiologically Maintained at Close to 50 Degrees C. PLOS Biol. 2018, 16, e2003992, DOI: 10.1371/journal.pbio.2003992.

18. Lane, N. Hot Mitochondria? PLOS Biol. 2018, 16, e2005113, DOI: 10.1371/journal.pbio.2005113. 
19. Suzuki, M.; Tseeb, V.; Oyama, K.; Ishiwata, S. Microscopic Detection of Thermogenesis in a Single Hela Cell. Biophys. J. 2007, 92, L46-L48, DOI 10.1529/biophysj.106.098673.

20. Yang, F.; Li, G.; Yang, J. M.; Wang, Z. H.; Han, D. H.; Zheng, F. J.; Xu, S. Y. Measurement of Local Temperature Increments Induced by Cultured Hepg2 Cells with Micro-Thermocouples in a Thermally Stabilized System. Sci Rep-Uk 2017, 7, 1721, DOI: 10.1038/s41598-017-01891-1.

21. Huang, H.; Delikanli, S.; Zeng, H.; Ferkey, D. M.; Pralle, A. Remote Control of Ion Channels and Neurons through Magnetic-Field Heating of Nanoparticles. Nat. Nanotechnol. 2010, 5, 602-606, DOI: 10.1038/Nnano.2010.125.

22. Jaque, D.; del Rosal, B.; Rodríguez, E. M.; Maestro, L. M.; Haro-González, P.; Solé, J. G. Fluorescent Nanothermometers for Intracellular Thermal Sensing. Nanomedicine 2014, 9, 1047-1062, DOI: 10.2217/Nnm.14.59.

23. Sakaguchi, R.; Kiyonaka, S.; Mori, Y. Fluorescent Sensors Reveal Subcellular Thermal Changes. Curr. Opin. Biotech. 2015, 31, 57-64, DOI: 10.1016/j.copbio.2014.07.013.

24. Carlos, L. D.; Palacio, F. Thermometry at the Nanoscale: Techniques and Selected Applications; Royal Society of Chemistry: Oxfordshire, 2016.

25. Quintanilla, M.; Liz-Marzan, L. M. Guiding Rules for Selecting a Nanothermometer. Nano Today 2018, 19, 126-145, DOI: 10.1016/j.nantod.2018.02.012.

26. Brites, C. D. S.; Balabhadra, S.; Carlos, L. D. Lanthanide-Based Thermometers: At the Cutting-Edge of Luminescence Thermometry. Adv. Opt. Mater. 2019, 7, 1801239, DOI: 10.1002/adom.201801239.

27. Nakano, M.; Nagai, T. Thermometers for Monitoring Cellular Temperature. J. Photoch. Photobio. C 2017, 30, 2-9, DOI: 10.1016/j.jphotochemrev.2016.12.001.

28. Freddi, S.; Sironi, L.; D'Antuono, R.; Morone, D.; Dona, A.; Cabrini, E.; D'Alfonso, L.; Collini, M.; Pallavicini, P.; Baldi, G., et al. A Molecular Thermometer for Nanoparticles for Optical Hyperthermia. Nano Lett. 2013, 13, 2004-2010, DOI: 10.1021/NI400129v.

29. Savchuk, O. A.; Silvestre, O. F.; Adão, R. M. R.; Nieder, J. B. Gfp Fluorescence Peak Fraction Analysis Based Nanothermometer for the Assessment of Exothermal Mitochondria Activity in Live Cells. Sci. Rep.-UK 2019, 9, 7535, DOI: 10.1038/s41598019-44023-7.

30. Okabe, K.; Inada, N.; Gota, C.; Harada, Y.; Funatsu, T.; Uchiyama, S. Intracellular Temperature Mapping with a Fluorescent Polymeric Thermometer and Fluorescence Lifetime Imaging Microscopy. Nat. Commun. 2012, 3, 705, DOI: 10.1038/ncomms1714.

31. Inada, N.; Fukuda, N.; Hayashi, T.; Uchiyama, S. Temperature Imaging Using a Cationic Linear Fluorescent Polymeric Thermometer and Fluorescence Lifetime Imaging Microscopy. Nat. Protoc. 2019, 14, 1293-1321, DOI: 10.1038/s41596-0190145-7.

32. Kalytchuk, S.; Polakova, K.; Wang, Y.; Froning, J. P.; Cepe, K.; Rogach, A. L.; Zboril, R. Carbon Dot Nanothermometry: Intracellular Photoluminescence Lifetime Thermal Sensing. ACS Nano 2017, 11, 1432-1442, DOI: 10.1021/acsnano.6b06670.

33. Yang, J. M.; Yang, H.; Lin, L. W. Quantum Dot Nano Thermometers Reveal Heterogeneous Local Thermogenesis in Living Cells. ACS Nano 2011, 5, 5067-5071, DOI: 10.1021/Nn201142f.

34. Vetrone, F.; Naccache, R.; Zamarron, A.; de la Fuente, Á. J.; Sanz-Rodríguez, F.; Maestro, L. M.; Rodríguez, E. M.; Jaque, D.; Solé, J. G.; Capobianco, J. A. Temperature Sensing Using Fluorescent Nanothermometers. ACS Nano 2010, 4, 3254-3258, DOI: 10.1021/Nn100244a.

35. Tanimoto, R.; Hiraiwa, T.; Nakai, Y.; Shindo, Y.; Oka, K.; Hiroi, N.; Funahashi, A. Detection of Temperature Difference in Neuronal Cells. Sci. Rep.-UK 2016, 6, 22071, DOI: 10.1038/srep22071. 
36. Liu, H. L.; Fan, Y. Y.; Wang, J. H.; Song, Z. S.; Shi, H.; Han, R. C.; Sha, Y. L.; Jiang, Y. Q. Intracellular Temperature Sensing: An Ultra-Bright Luminescent Nanothermometer with Non-Sensitivity to Ph and Ionic Strength. Sci. Rep-.UK 2015, 5 , 14879, DOI: 10.1038/srep14879.

37. Piñol, R.; Brites, C. D. S.; Bustamante, R.; Martínez, A.; Silva, N. J. O.; Murillo, J. L.; Cases, R.; Carrey, J.; Estepa, C.; Sosa, C., et al. Joining Time-Resolved Thermometry and Magnetic-Induced Heating in a Single Nanoparticle Unveils Intriguing Thermal Properties. ACS Nano 2015, 9, 3134-3142, DOI: 10.1021/acsnano.5b00059.

38. Zhu, X. J.; Li, J. C.; Qiu, X. C.; Liu, Y.; Feng, W.; Li, F. Y. Upconversion Nanocomposite for Programming Combination Cancer Therapy by Precise Control of Microscopic Temperature. Nat. Commun. 2018, 9, 2176, DOI 10.1038/s41467-01804571-4.

39. Qiu, X. C.; Zhou, Q. W.; Zhu, X. J.; Wu, Z. G.; Feng, W.; Li, F. Y. Ratiometric Upconversion Nanothermometry with Dual Emission at the Same Wavelength Decoded Via a Time-Resolved Technique. Nat. Commun. 2020, 11, 4, DOI 10.1038/s41467-019-13796-w.

40. Zhu, X.; Feng, W.; Chang, J.; Tan, Y.-W.; Li, J.; Chen, M.; Sun, Y.; Li, F. Temperature-Feedback Upconversion Nanocomposite for Accurate Photothermal Therapy at Facile Temperature. Nat. Commun. 2016, 7, 10437, DOI 10.1038/ncomms10437.

41. Malta, O. L. Ligand-Rare-Earth Ion Energy Transfer in Coordination Compounds. A Theoretical Approach. J. Lumin. 1997, 71, 229-236, DOI 10.1016/S0022-2313(96)00126-3.

42. Silva, F. R. G. E.; Malta, O. L. Calculation of the Ligand-Lanthanide Ion Energy Transfer Rate in Coordination Compounds: Contributions of Exchange Interactions. $J$. Alloy. Compd. 1997, 250, 427-430, DOI: 10.1016/S0925-8388(96)02563-7.

43. Malta, O. L.; Silva, F. R. G. E. A Theoretical Approach to Intramolecular Energy Transfer and Emission Quantum Yields in Coordination Compounds of Rare Earth Ions. Spectrochim. Acta A 1998, 54, 1593-1599, DOI: 10.1016/S1386-1425(98)000869.

44. Malta, O. L. Mechanisms of Non-Radiative Energy Transfer Involving Lanthanide Ions Revisited. J. Non-Cryst. Solids 2008, 354, 4770-4776, DOI: 10.1016/j.jnoncrysol.2008.04.023.

45. Carneiro Neto, A. N.; Teotonio, E. E. S.; de Sá, G. F.; Brito, H. F.; Legendziewicz, J.; Carlos, L. D.; Felinto, M. C. F. C.; Gawryszewska, P.; Moura, R. T.; Longo, R. L., et al. Modeling Intramolecular Energy Transfer in Lanthanide Chelates: A Critical Review and Recent Advances. In Handbook on the Physics and Chemistry of Rare Earths; Bünzli, J.-C. G.; Pecharsky, V. K., Eds.; Elsevier: 2019; Vol. 56, pp 55162.

46. Berry, J.; Weber, S. C.; Vaidya, N.; Haataja, M.; Brangwynne, C. P. Rna Transcription Modulates Phase Transition-Driven Nuclear Body Assembly. P. Natl. Acad. Sci. USA 2015, 112, E5237-E5245, DOI: 10.1073/pnas.1509317112.

47. Boisvert, F. M.; van Koningsbruggen, S.; Navascues, J.; Lamond, A. I. The Multifunctional Nucleolus. Nat. Rev. Mol. Cell. Bio. 2007, 8, 574-585, DOI: 10.1038/nrm2184. 
Figures
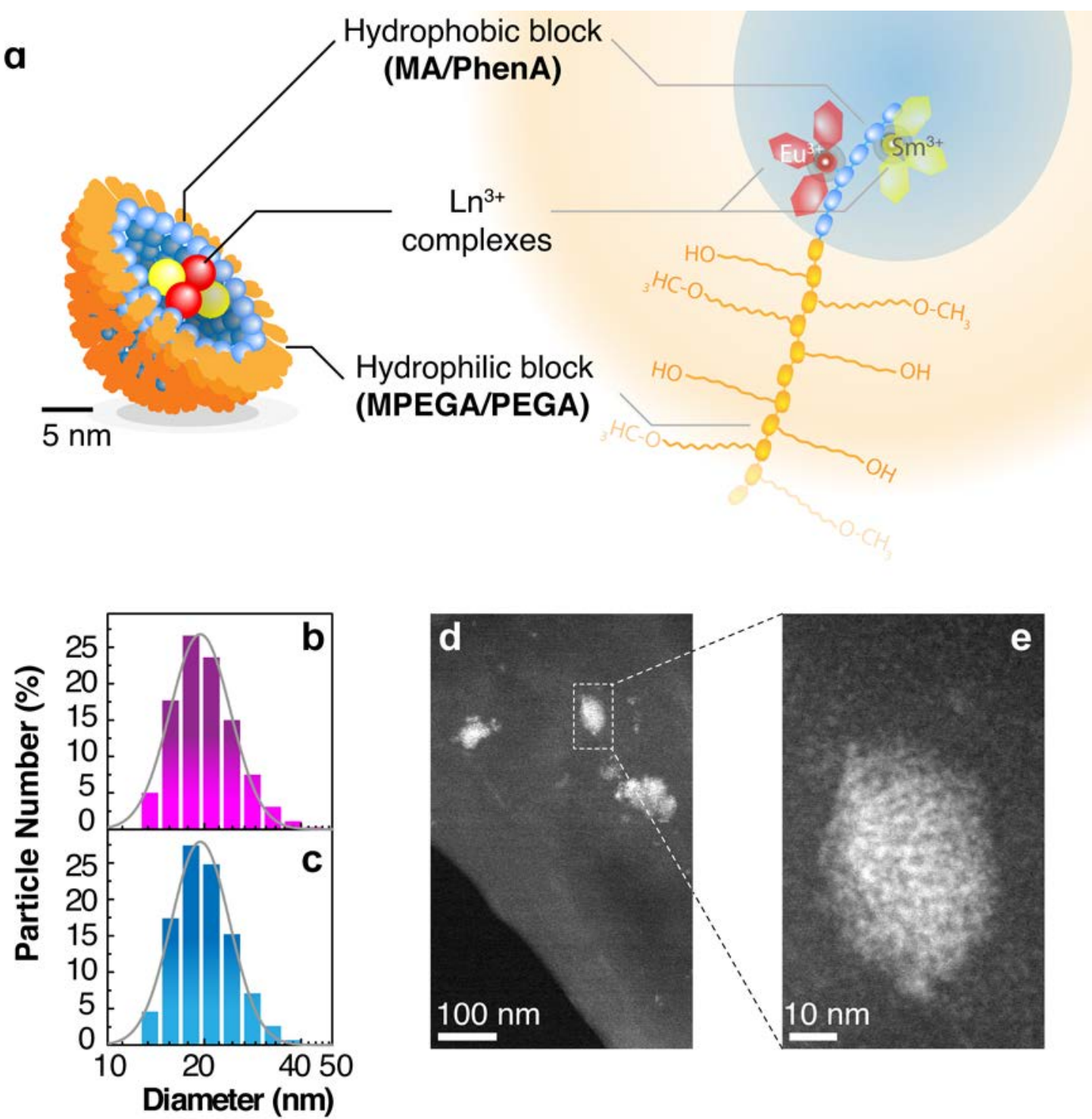

Figure 1. (a) Scheme of the $\mathrm{Ln}^{3+}$-bearing polymeric micelles. The details of the hydrophobic and hydrophilic polymer chains are presented in Figure S1, while those of the $\mathrm{Ln}^{3+}$ complexes in Figures S4 and S5 in Supporting Information. Dynamic light scattering size distribution of the $\mathrm{Ln}^{3+}$-bearing (b) btfa- and (c) DNPD- based polymeric micelles after filtration through a $100 \mathrm{~nm}$ membrane filter. The solid lines are the best fit to log-normal distributions $\left(r^{2}>0.99\right)$ centered at $20.3 \pm 0.2 \mathrm{~nm}$ in both cases. (d,e) Scanning transmission electron microscope images of the $\mathrm{Ln}^{3+}$-bearing btfa-based polymeric micelles. 


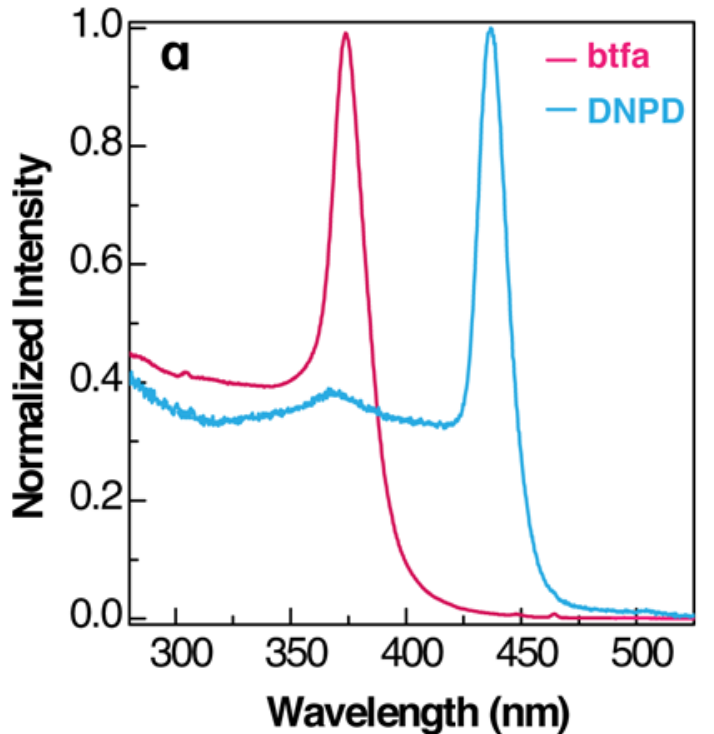

C

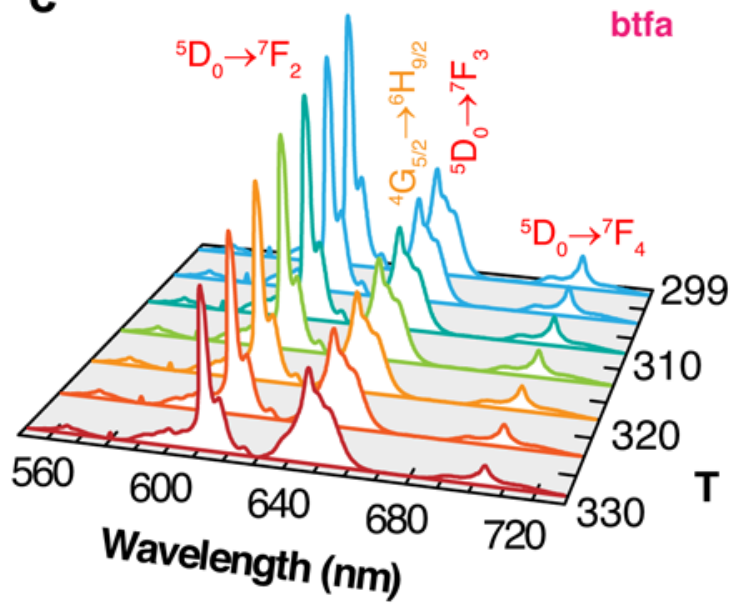

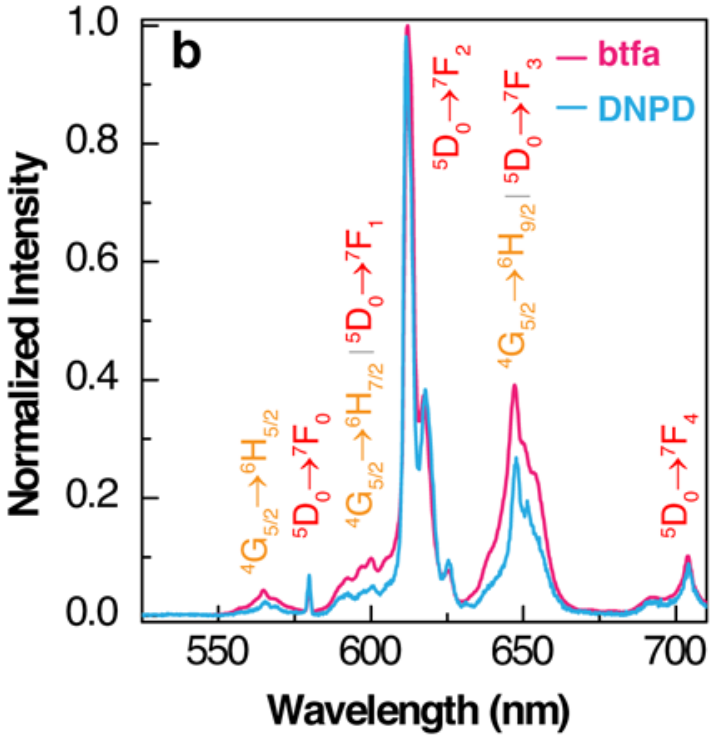

d

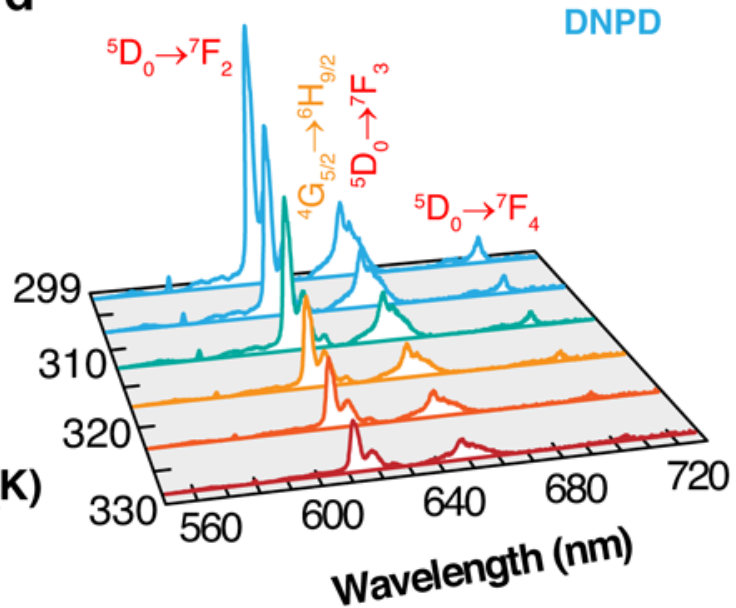

Figure 2. (a) Excitation spectra of btfa- and DNPD-based polymeric micelles at room temperature monitoring the ${ }^{5} \mathrm{D}_{0} \rightarrow{ }^{7} \mathrm{~F}_{2}$ transition of $\mathrm{Eu}^{3+}$. (b) Room temperature emission spectra of the btfa- and DNPD-based polymeric micelles. The transitions ascribed to $\mathrm{Eu}^{3+}$ and $\mathrm{Sm}^{3+}$ are signed in red and orange, respectively. Temperature dependence of the emission spectra recorded in the 299-328 K range for (c) btfa- and (d) DNPDbased polymeric micelles. In (b), (c) and (d) the excitation wavelengths used are 373 $\mathrm{nm}$ and $436 \mathrm{~nm}$ for btfa- and DNPD-based micelles, respectively. 

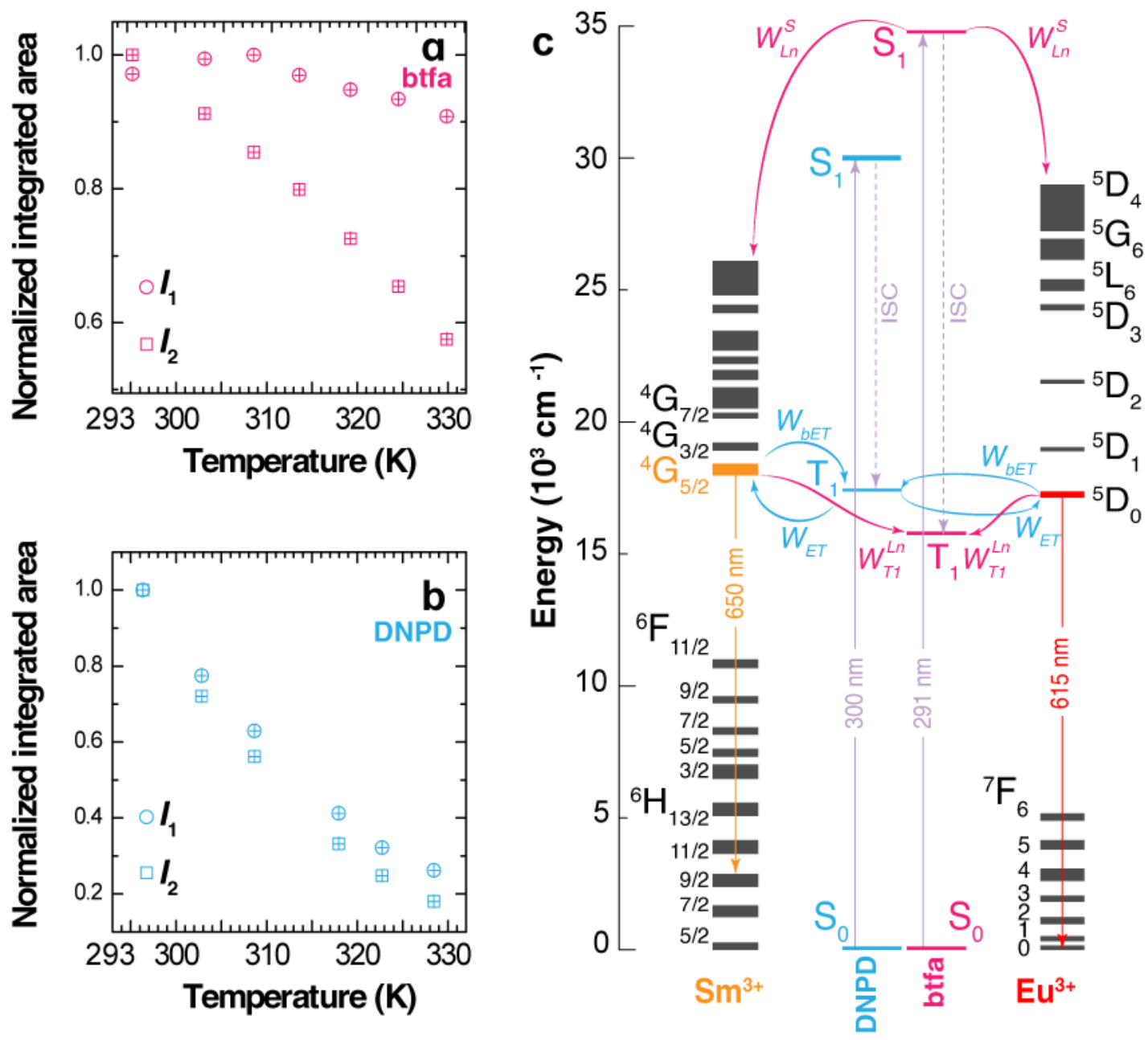

Figure 3. Temperature dependence of the integrated areas of the $\mathrm{Sm}^{3+}{ }^{4} \mathrm{G}_{5 / 2} \rightarrow{ }^{6} \mathrm{H}_{9 / 2}\left(I_{1}\right)$ and the $\mathrm{Eu}^{3+}{ }^{5} \mathrm{D}_{0} \rightarrow{ }^{7} \mathrm{~F}_{2}\left(I_{2}\right)$ transitions in the $\mathrm{Ln}^{3+}$-bearing (a) btfa- and (b) DNPD-based polymeric micelles. (c) Simplified energy level diagram for $\mathrm{Sm}^{3+}, \mathrm{Eu}^{3+}$, and ligands. The upwards arrow represents the initial absorptions by the ligands and ISC the intersystem crossing. For DNPD ligand, the forward intramolecular energy transfer rates $\left(W_{\mathrm{ET}}\right)$ are predominant via the $\mathrm{T}_{1} \rightarrow{ }^{4} \mathrm{G}_{5 / 2}\left(\mathrm{Sm}^{3+}\right)$ and $\mathrm{T}_{1} \rightarrow{ }^{5} \mathrm{D}_{0}\left(\mathrm{Eu}^{3+}\right)$ pathways. The backward energy transfer rates $\left(W_{\mathrm{bET}}\right)$ have a linear dependence with the temperature (Figure S6 in Supporting Information). For the btfa ligand, the forward intramolecular energy transfer rates $\left(\mathbf{W}_{\mathbf{L n}}^{\mathbf{S}}\right)$ occur essentially via the $\mathrm{S}_{1} \rightarrow{ }^{4} \mathrm{~F}_{5 / 2}\left(\mathrm{Sm}^{3+}\right)$ and $\mathrm{S}_{1} \rightarrow{ }^{5} \mathrm{G}_{2}$ $\left(\mathrm{Eu}^{3+}\right)$ pathways. In this case, the luminescence quenching depends on the energy balance between the transfer processes $\left(\mathbf{W}^{*}\right)$, because the energy turns back to the ligand via the acceptor state $T_{1}$. 

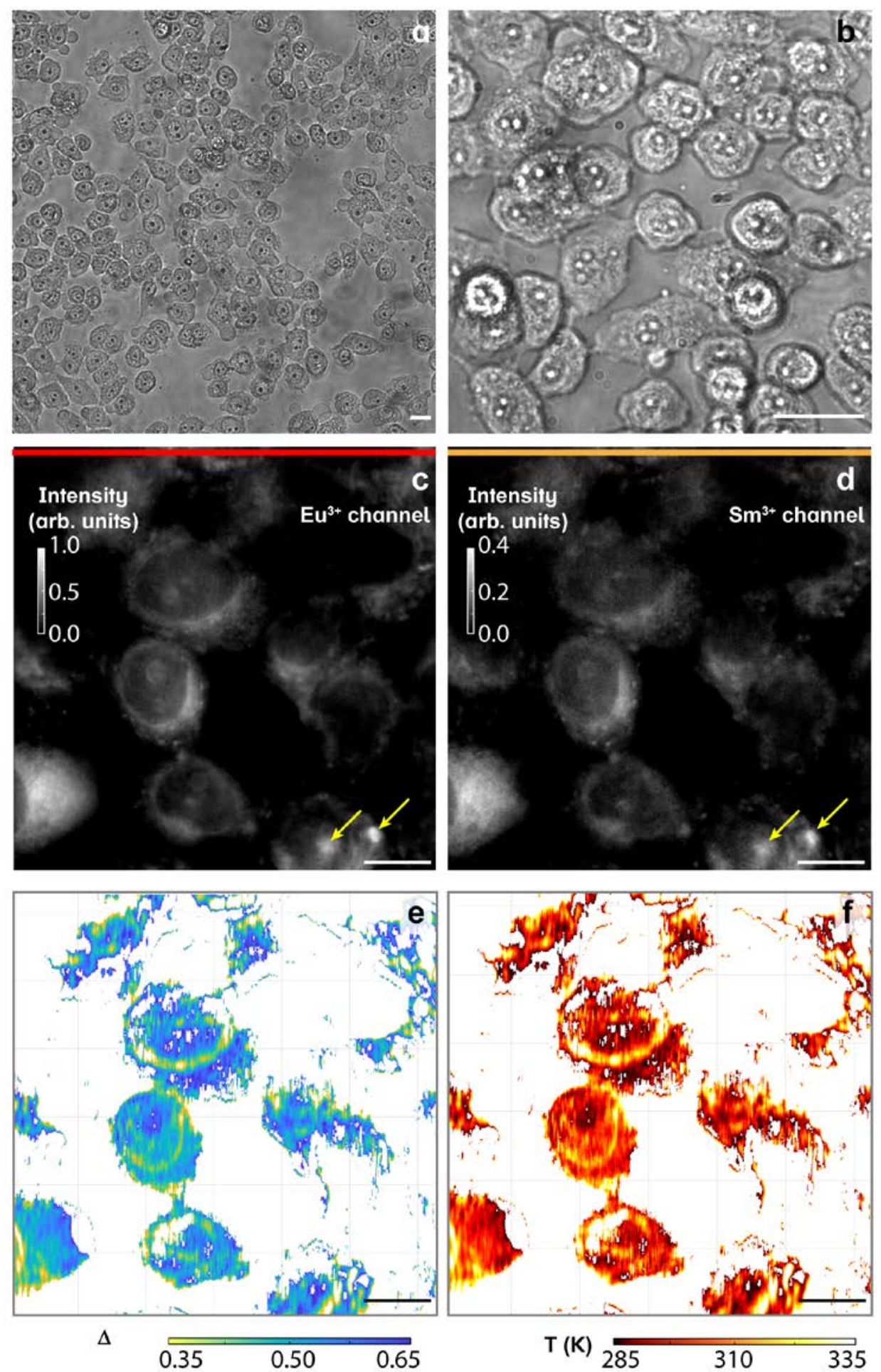

Figure 4. Room temperature microscopy images of MDA-MB-468 cells incubated with DNPD-based polymeric micelles under white-light illumination in (a) phase-contrast mode recorded with the color camera and (b) reflection mode recorded with the CMOS camera. Emission in the (c) $\mathrm{Eu}^{3+}$ and (d) $\mathrm{Sm}^{3+}$ channels recorded with the CMOS camera and illuminating the cultures with a $365 \mathrm{~nm}$ LED. The yellow arrows sign illustrative brighter regions of the cells. Calculated 2D (e) $\Delta$ (using $I_{1}$ and $I_{2}$ channel images) and (f) temperature maps. All the scale bars correspond to $10 \mu \mathrm{m}$. 

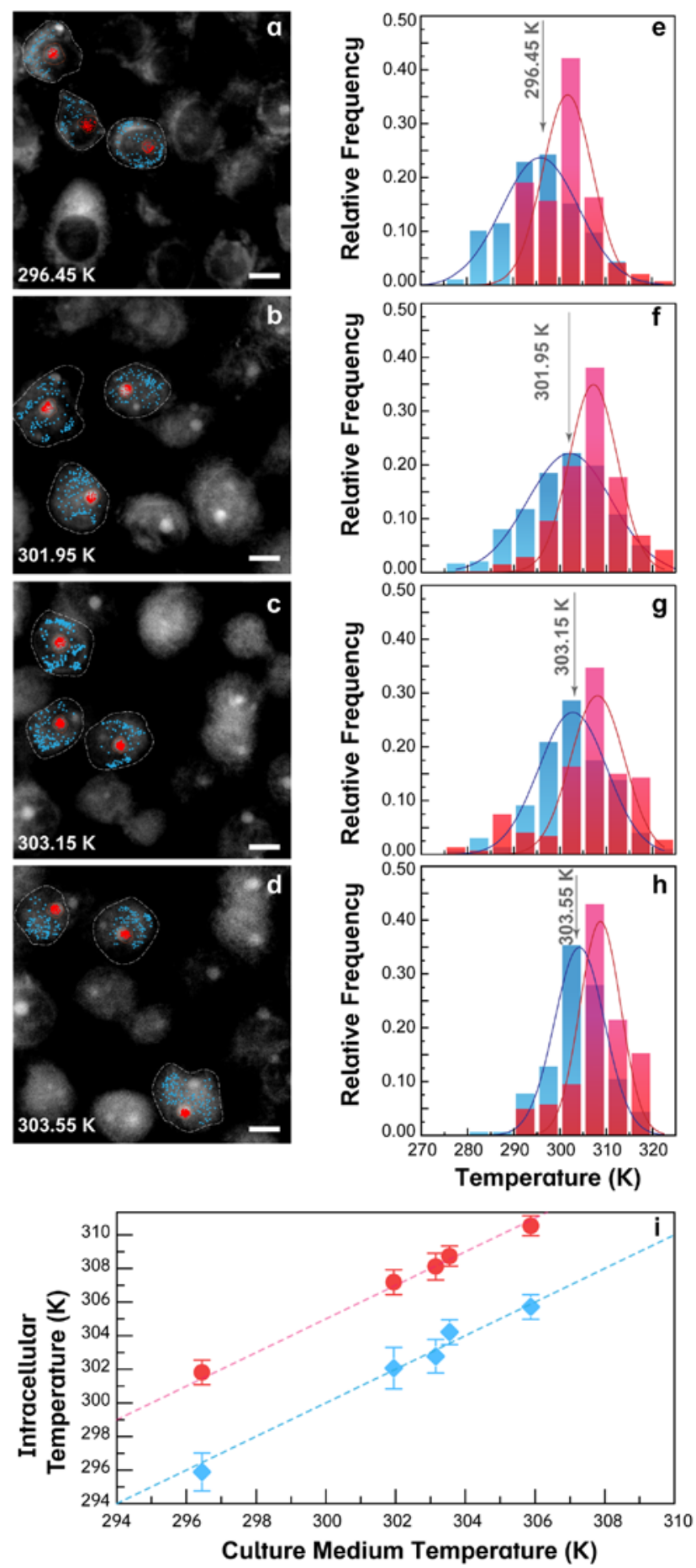

Figure 5. (a-d) Microscopy images (recorded in the $\mathrm{Eu}^{3+}$ channel) of the MDA-MB-468 cells incubated with DNPD-based polymeric micelles. The blue and red points mark, respectively, the locations used for temperature determination of the darker and brighter regions. The culture medium temperature values are indicated and The scale bars correspond to $10 \mu \mathrm{m}$. (e-h) Temperature histograms obtained from the selected points indicated in (a-d). The blue and red colors correspond to the blue (dark regions) and red (bright regions) points, respectively and the solid lines represent the best fits to Gaussian functions $\left(r^{2}>0.90\right)$. (i) Temperature determined using the intracellular thermometer $(y)$ versus culture medium temperature $(x)$ for the dark (blue) and bright (red) regions (e-h histograms). The blue line corresponds to $y=x$ and the red one is shifted by 5 degrees (guides for the eyes). Data for $305.95 \mathrm{~K}$ is presented in Figure S16 in Supporting Information. 


\section{Real-time intracellular temperature imaging using lanthanide-bearing polymeric micelles}

Rafael Piñol ${ }^{1}$, Justyna Zeler ${ }^{2,3}$, Carlos D. S. Brites ${ }^{2}$, Yuanyu Gu $u^{1,4}$, Pedro Téllez ${ }^{5}$, Albano N. Carneiro Neto ${ }^{2}$, Thiago E. da Silva ${ }^{2,6}$, Raquel Moreno-Loshuertos ${ }^{7}$, Patricio Fernandez-Silva ${ }^{7}$, Ana Isabel Gallego ${ }^{7}$, Luis Martinez-Lostao ${ }^{7}$, Abelardo Martínez ${ }^{8}$, Luís D. Carlos ${ }^{2 *}$, Angel Millán ${ }^{1^{*}}$

${ }^{1}$ ICMA, Institute of Materials Science of Aragon, CSIC-University of Zaragoza, 50008 Zaragoza, Spain

2 Phantom-g, CICECO-Aveiro Institute of Materials, Department of Physics, University of Aveiro, Campus de Santiago, 3810-193 Aveiro, Portugal

${ }^{3}$ Faculty of Chemistry, University of Wroclaw, Wroclaw, Poland

${ }^{4}$ School of Materials Science and Engineering. Nanjing Tech University, 210009, Nanjing PR China

${ }^{5}$ Servicio de Apoyo a la Investigación. University of Zaragoza, C/ Pedro Cerbuna 10, 50006 Zaragoza, Spain

6 Department of Fundamental Chemistry, Federal University of Pernambuco, 50670-901, Recife, PE, Brazil.

7 Departamento de Bioquímica, Biología Molecular y Celular, Universidad de Zaragoza, Zaragoza, Spain

${ }^{8}$ Departamento de Electrónica de Potencia, I3A, Universidad de Zaragoza 50018 Zaragoza, Spain 


\section{Contents}

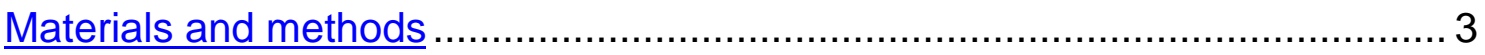

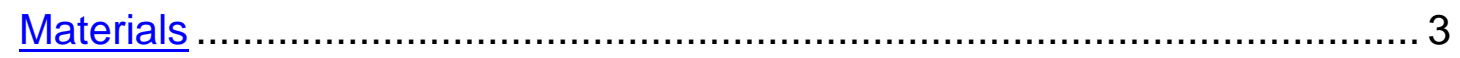

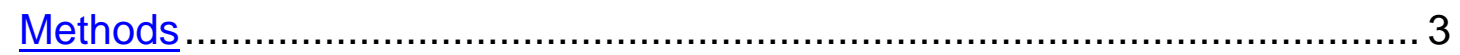

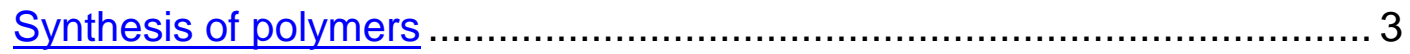

Characterization of polymers................................................................. 4

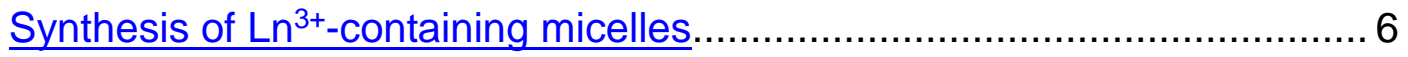

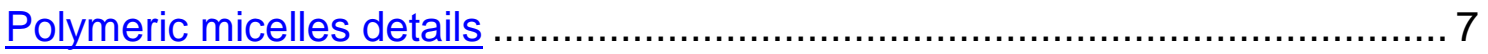

Chemical-physical characterization of the micellar solutions........................ 7

Stability of the micellar thermometric probes........................................... 9

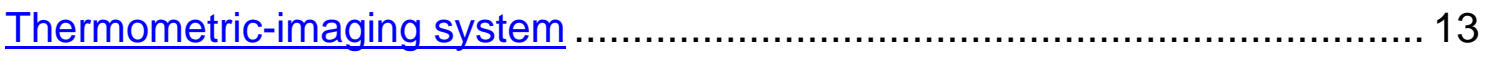

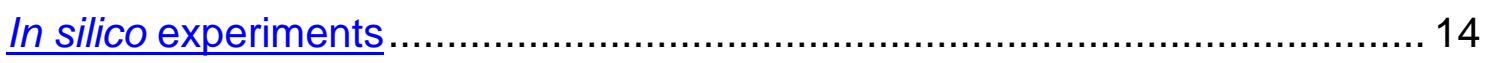

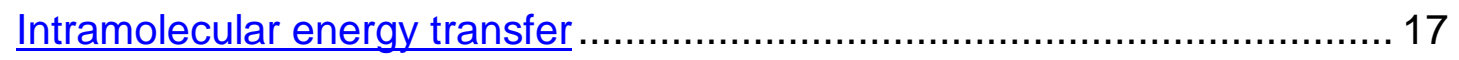

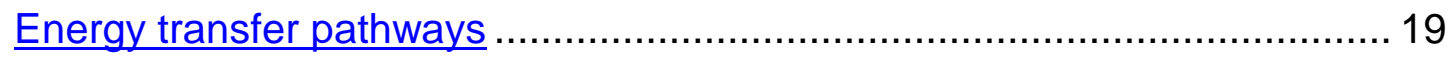

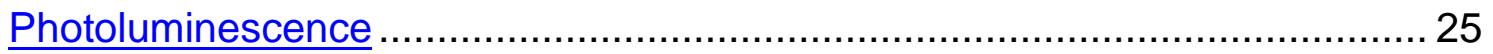

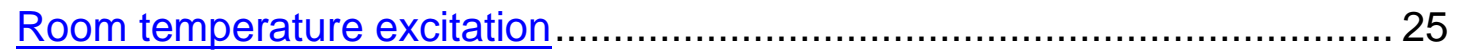

Thermometric performance of the $\mathrm{Ln}^{3+}$-containing micelles......................... 26

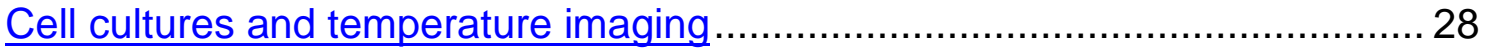

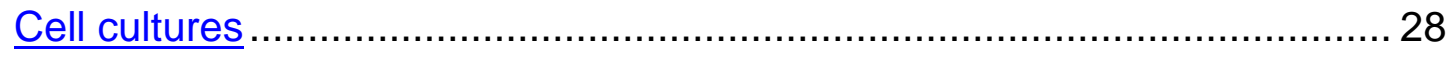

Preparation of cell samples for temperature imaging .................................. 28

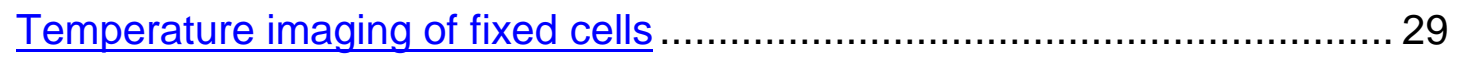

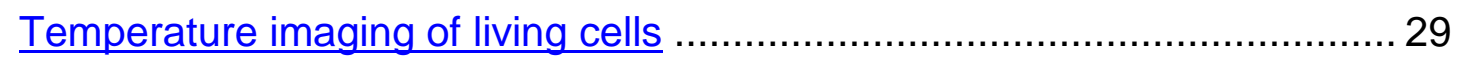

Optical properties of DNPD-based polymeric micelles in cell culture medium

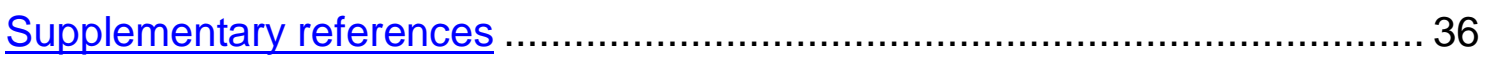




\section{Materials and methods}

\section{Materials}

Selenium dioxide $\left(\mathrm{SeO}_{2}, 99.999 \%\right)$ was purchased from Strem Chemicals. Europium(III) chloride hexahydrate $\left(\mathrm{EuCl}_{3}\left(\mathrm{H}_{2} \mathrm{O}\right)_{6}, 99.99 \%\right)$, samarium(III) chloride hexahydrate $\left(\mathrm{SmCl}_{3}\left(\mathrm{H}_{2} \mathrm{O}\right)_{6}, \quad 99.99 \%\right)$, 4,4,4-trifluoro-1-phenyl-1,3butanedione (btfa, 99\%) and 2-cyano-2-propyl dodecyl trithiocarbonate (97\%, RAFT chain transfer agent) were all products of Sigma Aldrich and were used as received without further purification. 4-Methyl-1,10-phenanthroline was synthesized according to the method described by Belser et al. ${ }^{1}$ The synthesis of 1,10-phenanthroline-4-carboxyaldehyde and 4-(hydroxymethyl)-1,10phenantroline was accomplished following the straightforward methods reported in the literature with slight modifications..$^{2-4}$

\section{Methods}

\section{Synthesis of polymers}

2,2'-Azobis(2-methylpropionitrile) (AIBN, Acros Organics, 98\%) was recrystallized twice from ethanol. Methyl acrylate (MA, 99\%, Aldrich) and poly(ethylene glycol) methyl ether acrylate (MPEGA) (Mn: $480 \mathrm{Da}$, Aldrich) was passed through a column of aluminum oxide to remove the inhibitors. Poly(ethylene glycol) acrylate (PEGA, Mn: $468 \mathrm{D}$, calculated by ${ }^{1} \mathrm{H}-\mathrm{NMR}$ spectroscopy) was synthesized from polyethylene glycol 400 (Aldrich) and purified according to procedures previously described. ${ }^{5}$ Polymerizable ancillary ligand 4-(acryloyloxymethyl)-1,10-phenanthroline (PhenA) was prepared by esterification reaction of (3) with acryloyl chloride.

Macro chain transfer agent (CTA) P(MPEGA-co-PEGA) and the final diblock copolymer, P(PMEGA-co-PEGA)-b-P(MA-co-PhenA), were prepared using reversible addition-fragmentation chain transfer (RAFT) polymerization. In the preparation of the P(MPEGA-co-PEGA)-b-P(MA-co-PhenA), P(MPEGA-coPEGA) was firstly synthesized using 2-cyano-2-propyl dodecyl trithiocarbonate as chain transfer agent, toluene as solvent, and AIBN as a radical source. The 
polymerization of the final polymer was carried out in dioxane as solvent adapting the method used for the synthesis of the MacroCTA. The structure of the final polymer is shown in Figure S1.

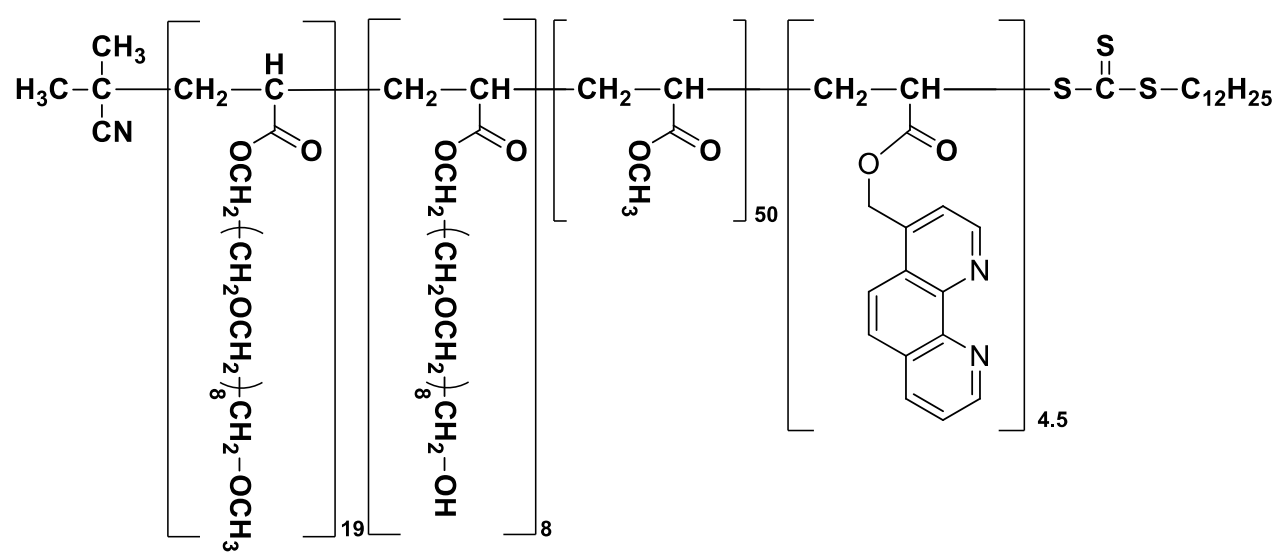

Figure S1. Structure of the block copolymer used in the fabrication of the thermometric micelles.

\section{Characterization of polymers}

\section{Nuclear Magnetic Resonance}

Proton $(1 \mathrm{H})$, and carbon (13C) NMR and NMR spectra were recorded at room temperature in a BRUKER AV-400 spectrometer (400 MHz (1H), $100 \mathrm{MHz}(13 \mathrm{C})$ using $\mathrm{CDCl} 3$ or DMSO-d6 as solvent. $1 \mathrm{H}$ and $13 \mathrm{C}$ chemical shifts $(\delta)$ are reported in ppm and are referenced to the solvent peak the coupling constants $(\mathrm{J})$ are given in $\mathrm{Hz}$. 1H-NMR spectroscopy was used to confirm the synthesis of P(MPEGA-Co-PEGA) and P(MPEGA-co-PEGA)-b-P(MA-PhenA) and determine the number average molecular weight, Mn (NMR), monomer percentage conversion, and composition of the polymers (degree of polymerization (DP), Mole fraction $(X)$ and Weight fraction $\left(X_{w}\right)$ for each monomer, Table S1). The calculated final molecular weight and the degree of polymerization were in concordance with the designed values.

\section{Size exclusion chromatography}

Molecular weights (Mn, Mw) and polydispersity (Mw/Mn) were measured by size exclusion chromatography using a Waters 2695 liquid chromatography system equipped with a Waters 2420 evaporation light scattering detector using a combination of two Styragel columns HR1 and HR4 calibrated using 
poly(methylmethacrylate, PMMA) standards and THF as solvent at a flow rate of $1 \mathrm{~mL}$ per minute at $35^{\circ} \mathrm{C}(308 \mathrm{~K})$.

Size-exclusion chromatograms (SEC) of both Macro Chain Transfer agent (CTA) P(MPEGA-co-PEGA) and the final diblock copolymer, P(PMEGA-coPEGA)-b-P(MA-co-PhenA) showed a narrow polymer distribution indicating the formation of well-defined polymers with good polydispersity.

Molecular weight and molecular weight distribution and composition of the block copolymer and the macroCTA measured by ${ }^{1} \mathrm{H}$ NMR spectroscopy and SEC are listed in Table S1.

Table S1. Molecular weight and composition of the block copolymer P(MPEGA-coPEGA)-b-P(MA-co-PhenA) and its macroCTA P(MPEGA-co-PEGA) determined by SEC or by NMR.

\begin{tabular}{|c|c|c|c|c|c|c|c|}
\hline Polymer & $\begin{array}{l}\text { Mn(Da) } \\
\text { (NMR) }\end{array}$ & $\begin{array}{l}\text { DP } \\
\text { NMR }\end{array}$ & $\begin{array}{l}X \\
\text { (NMR) }\end{array}$ & $\begin{array}{l}\text { Xw } \\
\text { (NMR) }\end{array}$ & $\begin{array}{l}\text { Mw(Da) } \\
\text { (SEC) }\end{array}$ & $\begin{array}{l}\text { Mn(Da) } \\
\text { (SEC) }\end{array}$ & $\begin{array}{l}\text { PDI } \\
\text { (SEC) }\end{array}$ \\
\hline \multirow{2}{*}{$\begin{array}{l}\text { P(MPEGA- } \\
\text { co-PEGA) }\end{array}$} & \multirow{2}{*}{13200} & $\begin{array}{l}\text { MPEGA } \\
\text { (19) }\end{array}$ & $\begin{array}{l}\text { MPEGA } \\
(0.70)\end{array}$ & $\begin{array}{l}\text { MPEGA } \\
(0.63)\end{array}$ & \multirow{2}{*}{9436} & \multirow{2}{*}{8752} & \multirow{2}{*}{1.08} \\
\hline & & $\begin{array}{l}\text { PEGA } \\
(8) \\
\text { MPEGA } \\
(19)\end{array}$ & $\begin{array}{l}\text { PEGA } \\
(0.30) \\
\text { MPEGA } \\
(0.23)\end{array}$ & $\begin{array}{l}\text { PEGA } \\
(0.37) \\
\text { MPEGA } \\
(0.50)\end{array}$ & & & \\
\hline \multirow{3}{*}{$\begin{array}{l}\text { P(MPEGA- } \\
\text { co-PEGA) } \\
\text {-b- } \\
\text { P(MA-co- } \\
\text { PhenA) }\end{array}$} & & $\begin{array}{l}\text { PEGA } \\
\text { (8) }\end{array}$ & $\begin{array}{l}\text { PEGA } \\
(0.11)\end{array}$ & $\begin{array}{l}\text { PEGA } \\
(0.20)\end{array}$ & \multirow{3}{*}{8803} & \multirow{3}{*}{8184} & \multirow{3}{*}{1.07} \\
\hline & $18 / 00$ & $\begin{array}{l}\text { MA } \\
(50)\end{array}$ & $\begin{array}{l}\text { MA } \\
(0.61)\end{array}$ & $\begin{array}{l}\text { MA } \\
(0.23)\end{array}$ & & & \\
\hline & & $\begin{array}{l}\text { PhenA } \\
(4.5)\end{array}$ & $\begin{array}{l}\text { PhenA } \\
(0.05)\end{array}$ & $\begin{array}{l}\text { PhenA } \\
(0.06)\end{array}$ & & & \\
\hline
\end{tabular}

\section{Fourier-transform infrared spectroscopy}

FT-IR spectra were recorded in a Perkin Elmer Spectrum 100 FT-IR spectrometer equipped with a universal attenuated total reflection sampling accessory. The spectra were collected over the $4000--380 \mathrm{~cm}^{-1}$ range (attenuated total reflection correction performed).

The FTIR analysis confirmed the chemical structure of the block copolymer and the successful anchoring of the complex. The presence of lanthanide complexes was revealed by the presence of characteristic vibrational 1650-1500 $\mathrm{cm}^{-1}$ bands associated with the metal-ligand coordination bonds. In the case of 
the btfa complexes, the C-F stretching bands associated with the $\mathrm{CF}_{3}$ groups of the ligand can also be observed at $1132 \mathrm{~cm}^{-1}$. A comparison of the spectrum of polymer powders obtained by evaporation of the stock solutions in THF with that of micelles powders obtained by freeze-drying of the final micelle aqueous suspension showed no changes in the polymer bands, indicating that the integrity of complex was preserved during the auto self-assembly process of the polymer in water.

\section{Differential scanning calorimetry}

Thermal analysis was conducted by differential scanning calorimetry in a Q2000 TA equipment. The samples were quickly cooled down to $-40^{\circ} \mathrm{C}(233 \mathrm{~K})$ and then heated at the rate of $10 \mathrm{~K} \cdot \mathrm{min}^{-1}$.

The DSC traces of the P(MPEGA-Co-PEGA) MacroCTA were similar to those reported in the literature for P(MPEGA) homopolymers with a higher degree of polymerization. The crystallization of PEG side chains was observed at around $39^{\circ} \mathrm{C}$, and the melting peak was observed at nearly $-12^{\circ} \mathrm{C}$. However, the DSC traces of block copolymer showed neither crystallization peak nor Tg transition in the $-40^{\circ} \mathrm{C}$ to $60^{\circ} \mathrm{C}$ temperature range indicating that both blocks are in a soft state at these temperatures.

\section{Elemental analysis}

Elemental analysis for C, H were performed using a Perkin Elmer CHN 2400 elemental analyzer with standard combustion conditions and handling of the samples in the air.

\section{Synthesis of $\mathrm{Ln}^{3+}$-containing micelles}

1,3-di(naphthalen-2-yl)propane-1,3-dione (DNPD) was prepared by adapting the synthetic method described by McGehee et al. ${ }^{6}$ Europium and samarium complexes, denoted by $\left[\mathrm{Ln}(\mathrm{L})_{3}\left(\mathrm{H}_{2} \mathrm{O}\right)_{2}\right](\mathrm{Ln}=\mathrm{Eu}, \mathrm{Sm})$ were prepared following the synthetic procedure reported by Binnemans et al. ${ }^{7}$ The $\mathrm{Ln}(\mathrm{L})_{3} \cdot 2 \mathrm{H}_{2} \mathrm{O}$ complexes were covalently attached by coordination with the ancillary ligand included in the polymer chain (PhenA) in the following way: the block copolymer and $\mathrm{Ln}(\mathrm{L})_{3} \cdot 2 \mathrm{H}_{2} \mathrm{O}$ complexes were mixed in chloroform in stoichiometric proportion 
(PhenA: Ln, 1:1; $\mathrm{Eu}(\mathrm{L})_{3} \cdot 2 \mathrm{H}_{2} \mathrm{O}(7.5 \% \mathrm{~mol})$ and $\left.\mathrm{Sm}(\mathrm{L}) 3 \cdot 2 \mathrm{H}_{2} \mathrm{O}(92.5 \% \mathrm{~mol})\right)$ and heat overnight under reflux. After evaporation of the solvent, the polymer was dissolved in tetrahydrofuran (THF). Finally, the micelles were formed by selfassembly after the addition of water to the THF solution.

\section{Polymeric micelles details}

The hydrophilic block is a brush-like block of methoxy (MPEGA) and hydroxyl (PEGA) end-capped polyethylene glycol acrylates. The hydrophobic block is a methyl acrylate (MA) polymer containing a discrete number of the auxiliary ligand PhenA. The general formula of the lanthanide complexes is $L n(L 1){ }_{3} L 2$ where $L 1$ represents a $\beta$-diketonate ligand: 1,3-di(naphthalen-2-yl)propane-1,3-dione (DNPD) and L2 is an ancillary ligand, (1,10-phenanthrolin-4-yl)methyl acrylate (PhenA), a 1,10 phenanthroline derivative modified with a polymerizable acrylate group and pendant from the polymer chain after polymerization. The complexes are covalently attached to the polymer chain by coordination with the phenanthroline auxiliary ligand in chloroform. Then, the chloroform was evaporated, and the resulting product was dissolved in THF. Finally, the micelles are formed by self-assembly after the addition of water to the THF solution. The hydrophilic PEG block forms the outer shell of the micelles and the non-watersoluble methyl acrylate block containing the lanthanide complexes forms the inner shell. The presence of the PEG block provides simultaneously stability in aqueous media, biocompatibility, enhanced cellular uptake, and low toxicity to the nanostructures. Several batches of the btfa- and DNPD-based micelles were prepared with analogous structural and spectroscopic properties.

\section{Chemical-physical characterization of the micellar solutions}

\section{$\underline{\text { Inductively coupled plasma optical emission spectrometry }}$}

Analysis of thermometric micelles by inductively coupled plasma optical emission spectrometry in a plasma 40 ICP Perkin-Elmer spectrometer yielded a concentration of Eu and Sm in the micelle aqueous dispersion of $0.44 \times 10^{-4} \mathrm{~mol} / \mathrm{L}$, and $4.60 \times 10^{-4} \mathrm{~mol} / \mathrm{L}$, respectively, corresponding to an Eu/Sm molar ratio of 0.095. The samples for analysis were prepared by digestion of $1 \mathrm{~mL}$ of the micelle 
aqueous dispersion with concentrated $\mathrm{HNO}_{3}(1 \mathrm{~mL})$ overnight and then diluting to a final volume of $10 \mathrm{~mL}$ with ultrapure water.

\section{Dynamic light scattering and zeta potential}

Dynamic light scattering (DLS) and zeta potential measurements of the ferrofluids were performed on a Malvern Zetasizer NS (Malvern Instruments Ltd., Worcestershire, UK) using a He-Ne laser with a $633 \mathrm{~nm}$ wavelength and a detector angle of $173^{\circ}$. The measurements were repeated three times, and the average was calculated.

\section{$\underline{\text { Transmission electron microscopy }}$}

Transmission electron microscopy (TEM) imaging was performed in a FEl Tecnai F30 microscope equipped with an EDAX XEDS system with an energy resolution of $130 \mathrm{eV}$. Samples for TEM analysis were prepared by dip-coating on lacey carbon grids.

Energy dispersive X-ray spectroscopy (EDS) of Sm and Eu content was performed in selected areas within the thermometric micelles. The presence of $\mathrm{SmM}_{\alpha 1} \mathrm{SmL}_{\alpha 1}$ and $\mathrm{SmL}_{\beta 1,2}$ peaks was observed (Figure S2).
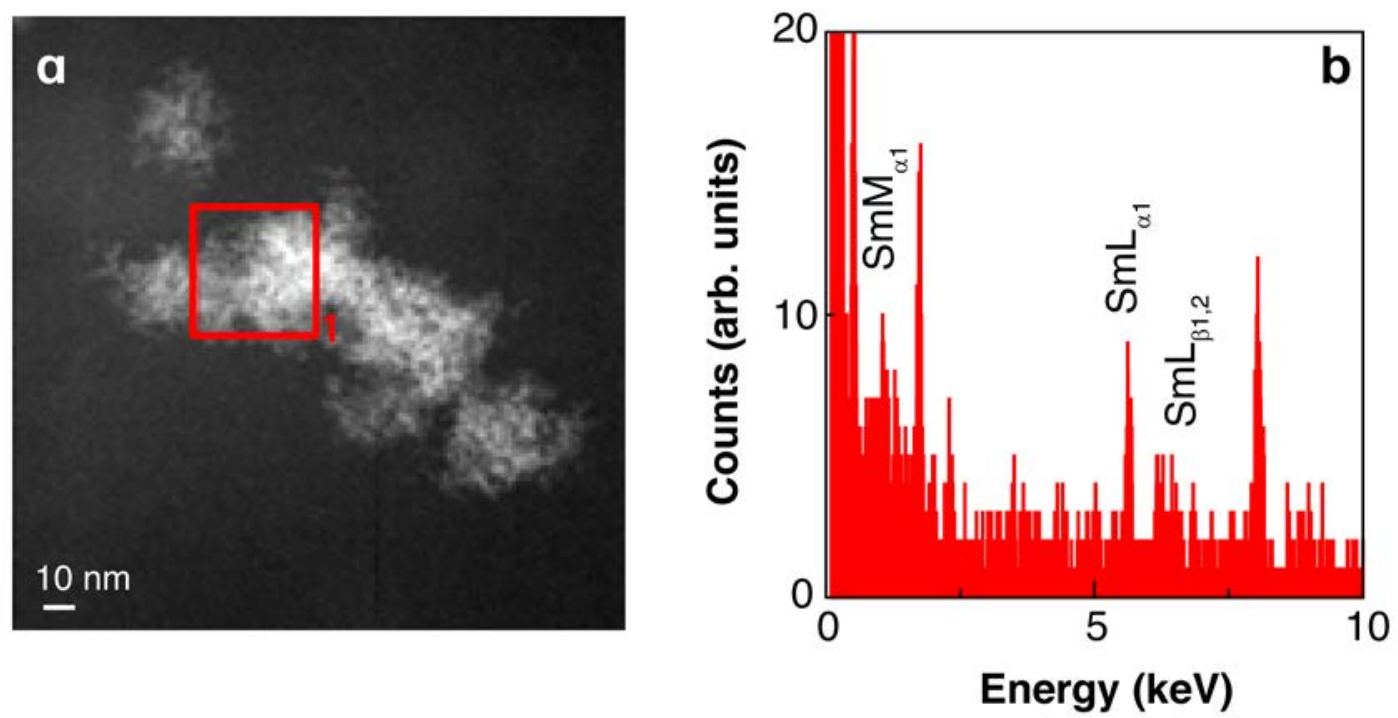

Figure S2. EDS analysis on an area of a sample prepared by evaporation of the ferrofluid on a carbon-coated copper grid. The Sm emission can be clearly distinguished. 


\section{$\underline{\text { Temperature-dependent photoluminescence }}$}

The photoluminescence spectra were measured in the room-temperature $300-328 \mathrm{~K}$ range with a modular double-grating excitation spectrofluorimeter with a TRIAX 320 emission monochromator (Fluorolog-3, Horiba Scientific) coupled to an R928 Hamamatsu photomultiplier using the front face acquisition mode. The excitation source was a $450 \mathrm{~W}$ Xe arc lamp. For the temperaturedependent experiments, the samples were in contact with a heating stage (Linkam, THMS 600).

\section{Stability of the micellar thermometric probes}

To ensure a firm attachment of the lanthanide emitters to the copolymer forming the micelles, we followed the strategy of fixing the lanthanide Ln(L1)3 complexes to the polymer backbone by covalent binding the complex (instead of physical absorption) with a strong lanthanide ligand, as phenanthroline, that is incorporated in the polymer structure. This strategy also produces an enhancement of the emission intensity, with respect to the pure $\operatorname{Ln}(\operatorname{Lr})_{3}$ complexes.

The attachment of the $\operatorname{Ln}(\mathrm{L})_{3}$ complexes to the phenanthroline ancillary ligand in the polymer was studied by Fourier transform infrared spectroscopy (FTIR) (Figure S3). The disappearance of the broad absorption bands centered at 3380 $\mathrm{cm}^{-1}$, corresponding to the $\mathrm{H}_{2} \mathrm{O}$ coordination molecules in the $\mathrm{Ln}(\mathrm{L})_{3}\left(\mathrm{H}_{2} \mathrm{O}\right)_{2}$ complexes, indicates the displacement of these molecules by coordination of the lanthanides to the phenanthroline ligand in both types of micelles. In the case of the btfa micelles, the FTIR spectrum shows a relative increase of the intensity of the $(C=O)$ stretching band at $1610 \mathrm{~cm}^{-1}$, with respect to that at $1597 \mathrm{~cm}^{-1}$, and a widening of the vibration frequency band at $1530 \mathrm{~cm}^{-1}$ typical of pure Ln(btfa) ${ }_{3}$ Phen complexes. In the case of DNPD micelles, an increase in the strength of the band at $1495 \mathrm{~cm}^{-1}$, and a shift of the $1375 \mathrm{~cm}^{-1}$ band to higher wavenumbers $\left(1404 \mathrm{~cm}^{-1}\right)$ are also evidence of lanthanide phenanthroline coordination bonds. 


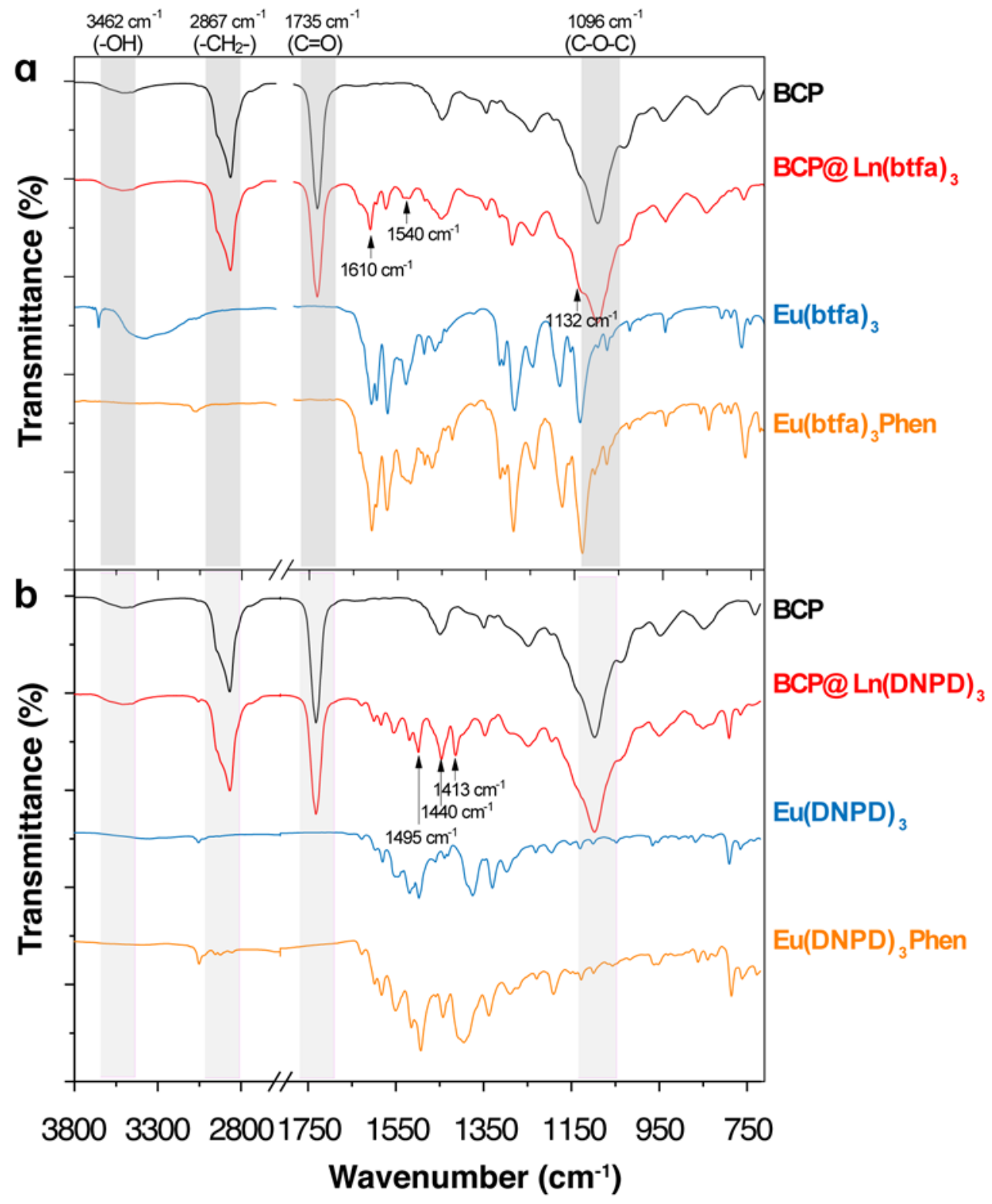

Figure S3. FTIR spectra for $P(M P E G A-c o-P E G A)-b-P(M A-b-P h e n A)$ (black line), P(MPEGA-co-PEGA)-b-P(MA-b-(PhenA@EuSm $\left.(\mathrm{L})_{3}\right)$ (red line), Eu(L) ${ }_{3}\left(\mathrm{H}_{2} \mathrm{O}\right)_{2}$ complex (blue line) and $\mathrm{Eu}(\mathrm{L})_{3}$ Phen (orange line) for (a) $\mathrm{L}=\mathrm{btfa}$ and (b) $\mathrm{L}=\mathrm{DNPD}$ based material.

Lanthanide metal content in the micellar suspension was determined by Inductively Coupled Plasma Atomic Emission Spectrometry (ICP-AES) in a plasma 40 ICP Perkin-Elmer spectrometer. Samples for ICP-AES were prepared by digestion of $1 \mathrm{~mL}$ of the micellar solution after freeze-drying with concentrated $\mathrm{HNO}_{3}$ overnight and diluted with ultrapure water. The experimental ratios of the $\mathrm{Ln}^{3+}$ ions were $2.42 \%$ and $2.84 \% \mathrm{w} / \mathrm{w}$, for DNDP and btfa complexes, respectively. Considering the nominal values of $2.84 \%$ and $3.03 \% \mathrm{w} / \mathrm{w}$ used in the encapsulation reaction, the loading efficiency was $85 \%$ and $94 \%$ for DNDP 
and btfa complexes, respectively. The stability of the micelles suspensions was studied by DLS (Figure S4). It was observed that the size remains unchanged at least 6 months after storage.

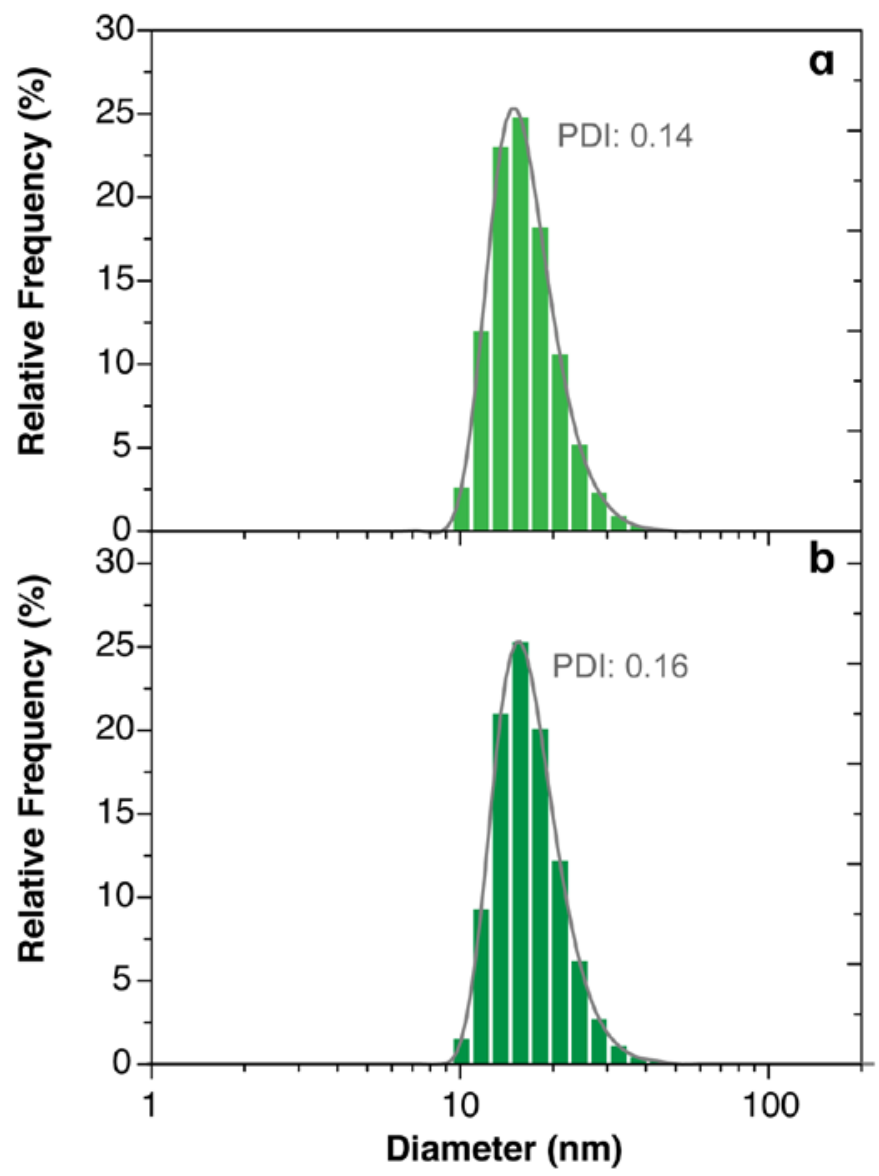

Figure S4 Histograms of the hydrodynamic diameter for btfa-based micelles measured (a) after 1 day and (b) after 6 months of the preparation.

The effect of dilution on the structural and optical stabilities of the micellar solutions was attested by DLS (Figure S5a) and photoluminescence measurements (Figure S5b). Dilutions in ultrapure water of the as-prepared solution by $1 / 5$ and $1 / 10$ did not cause any change neither in the size distribution nor in the emission spectra of the micelles. Moreover, the relative ratio of $\mathrm{Eu}^{3+} / \mathrm{Sm}^{3+}$ intensities was also maintained after dilution. 

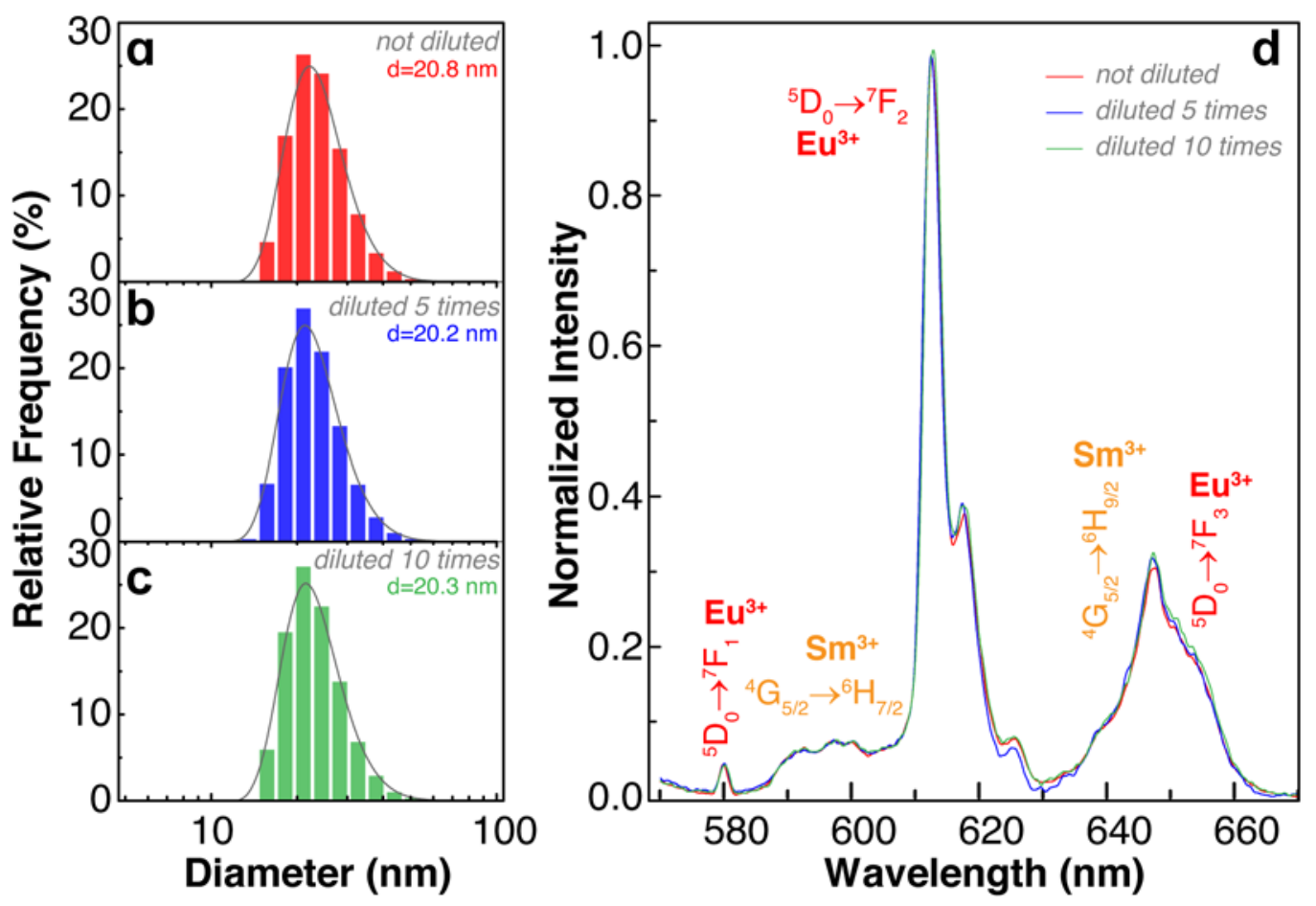

Figure S5 (a) Histograms of the hydrodynamic diameter (d) and (b) room-temperature emission spectra of btfa-based micelles under $365 \mathrm{~nm}$ excitation for different dilution factors.

The leakage of the btfa- and DNPD-based complexes from the micelles was checked using cryo-TEM. The fast freezing of the suspension in this technique ensures that the frozen sample precisely reproducing the state of the materials in the liquid. Cryo-STEM images together with electron energy loss spectroscopy (EELS) and EDS analysis of micelle samples several weeks after preparation showed no presence of lanthanides outside the micelles, demonstrating the absence of significant leaking from the interior of the micelles into the bulk of the suspension.

The optical properties of the $\mathrm{Ln}^{3+}$-bearing complexes changed in cell culture media with respect to pure water. It has experimentally observed that $\mathrm{Ln}(\mathrm{L} 1)_{3} \cdot 2 \mathrm{H}_{2} \mathrm{O}$ complexes lost their emission in the cell culture medium. The linking the $\mathrm{Ln}(\mathrm{L} 1)_{3} \cdot 2 \mathrm{H}_{2} \mathrm{O}$ complexes to the phenanthroline ligand protects from luminescence quenching effects by steric shielding. However, it was observed that the shielding effect was not sufficient in the case of $\operatorname{Ln}(\mathrm{btfa})_{3}$ Phen that decreased gradually its emission intensity when transferred from water to the cell culture medium. Nevertheless, when the bfta ligands were replaced by DNPD the luminescence was preserved in cell culture medium and persisted after 
internalization of the micelles in the cells. Such enhancement of the emission stability in the cell culture medium can be attributed to the improvement of the steric effects of the coordination shell due to the larger volume of the DNPD ligand as compared to the btfa one (Figure S6).

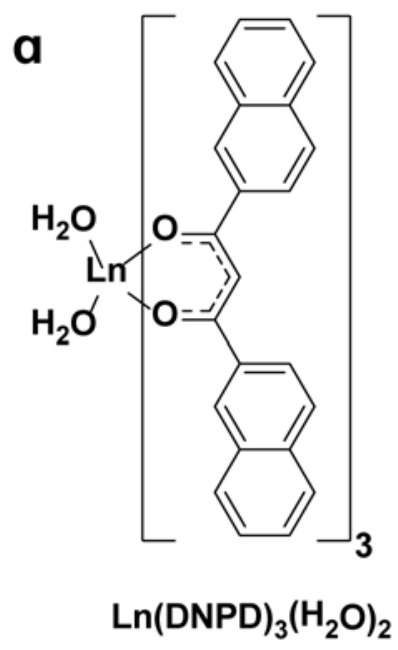

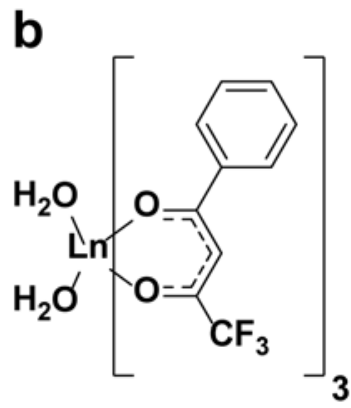

$\operatorname{Ln}(\mathrm{btfa})_{3}\left(\mathrm{H}_{2} \mathrm{O}\right)_{2}$

Figure S6. Structure of (a) $\operatorname{Ln}(\mathrm{DNPD})_{3}\left(\mathrm{H}_{2} \mathrm{O}\right)_{2}$ and (b) $\operatorname{Ln}(\mathrm{btfa})_{3}\left(\mathrm{H}_{2} \mathrm{O}\right)_{2}$ complexes.

\section{Thermometric-imaging system}

The fluorescence microscope used in cell imaging experiments was a Leica DMI3000B equipped with a DFC 7000 color camera. The pixel-to-pixel lateral resolution is $\sim 97 \mathrm{~nm}$, although the ultimate spatial resolution is determined by the microscope optics, about $1 \mu \mathrm{m}$. The beam splitter and the CMOS camera are from Hamamatsu instrument and a Leica fluorescence microscope incorporating a Gemini beam splitter and a CMOS Orca 4.0 camera (both from Hamamatsu) connected to a computer endowed with a LabView image-processing program. The beam splitter was equipped with a Semrock FF635-Di01 dichroic, an FBH650-40 bandpass filter, central wavelength $650 \mathrm{~nm}$, full-width at half maximum, fwhm=40 nm (for Sm ${ }^{3+}$ emission), and a Chroma ET610/20 NC491994 bandpass filter, central wavelength $610 \mathrm{~nm}$, fwhm=20 nm (for Eu ${ }^{3+}$ emission). 
a

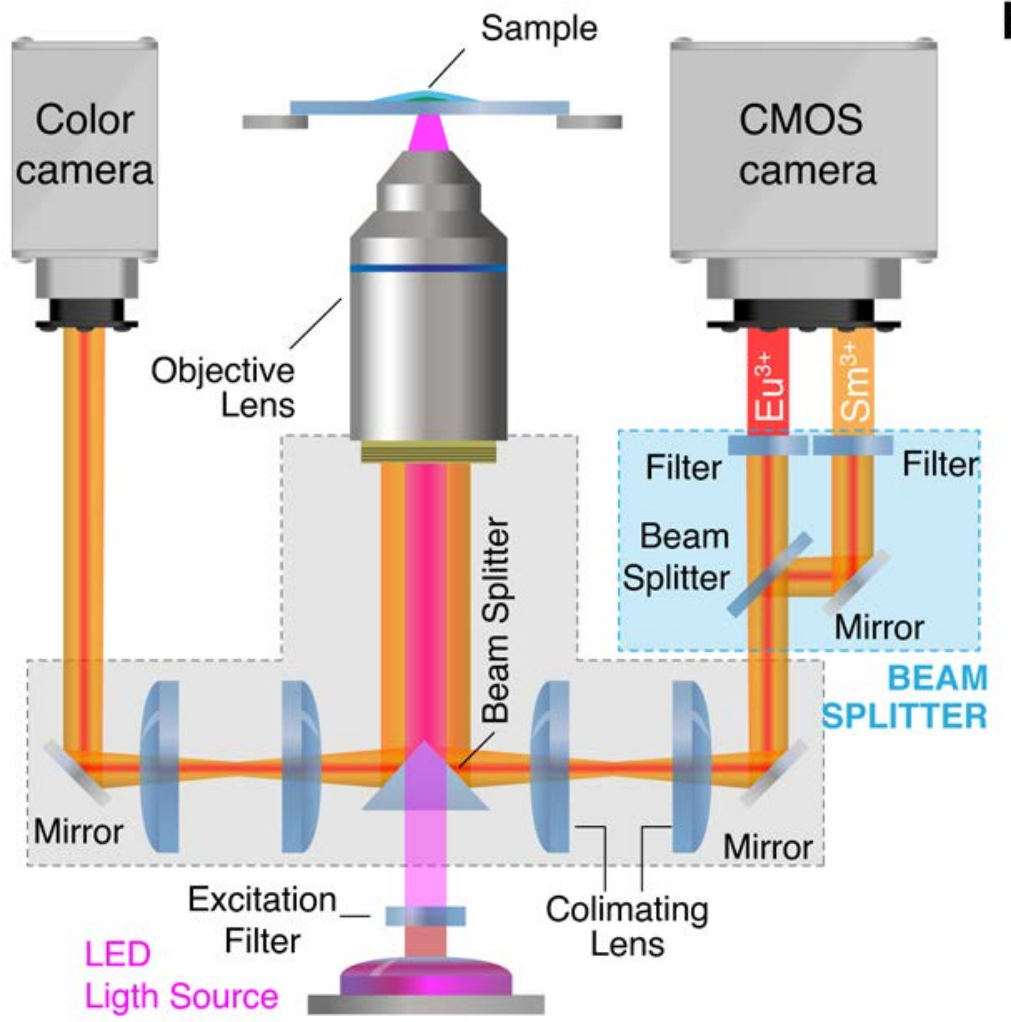

b

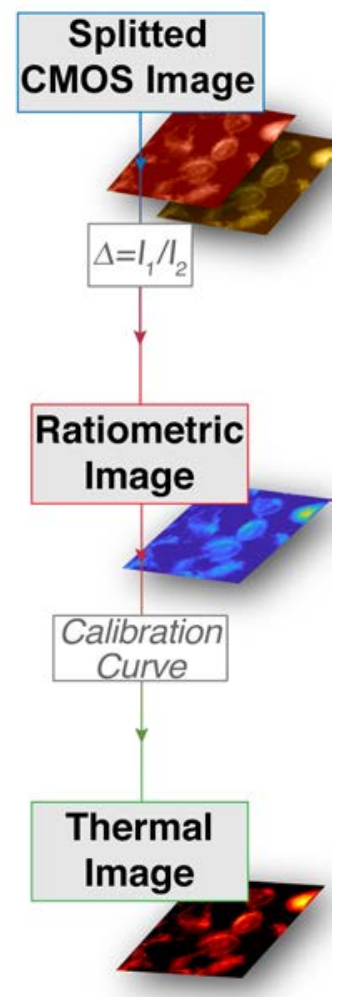

Figure S7. (a) Scheme of the microscope setup used to acquire simultaneously the regular color image (DFC 7000 color camera) and the split CMOS image (Orca 4.0 camera) for temperature mapping. The LED light $(365 \mathrm{~nm})$ passes the excitation filter before illuminating the sample. The dichroic mirror blocks the excitation light to prevent CMOS damage The emitted light is divided by a customized beam-splitter system that projects the $\mathrm{Sm}^{3+}(620-660 \mathrm{~nm})$ and the $\mathrm{Eu}^{3+}(600-620 \mathrm{~nm})$ spectral regions in each half of the CMOS camera detector. (b) Flux diagram of the image processing using the MatLab® routine.

\section{In silico experiments}

Since there are no $\mathrm{Gd}^{3+}$ (or even $\mathrm{La}^{3+}$ or $\mathrm{Lu}^{3+}$ ) samples to obtain the ligands excited energies $\mathrm{T}_{1}$ and $\mathrm{S}_{1}$, quantum chemistry calculations are useful to estimate these states. In this work, DFT and TD-DFT (time-dependent DFT) calculations, using Gaussian 09 program, ${ }^{8}$ were performed in the presence of a solvent (water) within the Polarizable Continuum Model (PCM) using the integral equation formalism variant (IEFPCM). ${ }^{9-11}$ For both, structure optimizations and excited states calculations were used $\omega B 97-X D$ functional. ${ }^{12}$ Due to a large number of atoms (> 200), the basis set STO-3G (for $\mathrm{C}, \mathrm{H}, \mathrm{N}$ and $\mathrm{O}$ atoms) was used while the $\mathrm{Ln}^{3+}$ ions were treated with MWB52 $\left(\mathrm{Eu}^{3+}\right)$ and MWB51 $\left(\mathrm{Sm}^{3+}\right) .^{13}$ 
There is no significant difference in structures between $\mathrm{Eu}^{3+}$-DNPD and $\mathrm{Sm}^{3+}$ DNPD coordination compounds. Figure S8a shows the structure from DFT simulations, the highlighted atoms (white spheres) represent the ancillary ligand bonded with the polymer. Figure S8b-c shows the main compositions of molecular orbitals (from occupied to unoccupied ones) involved in the $\mathrm{T}_{1}$ excited state.
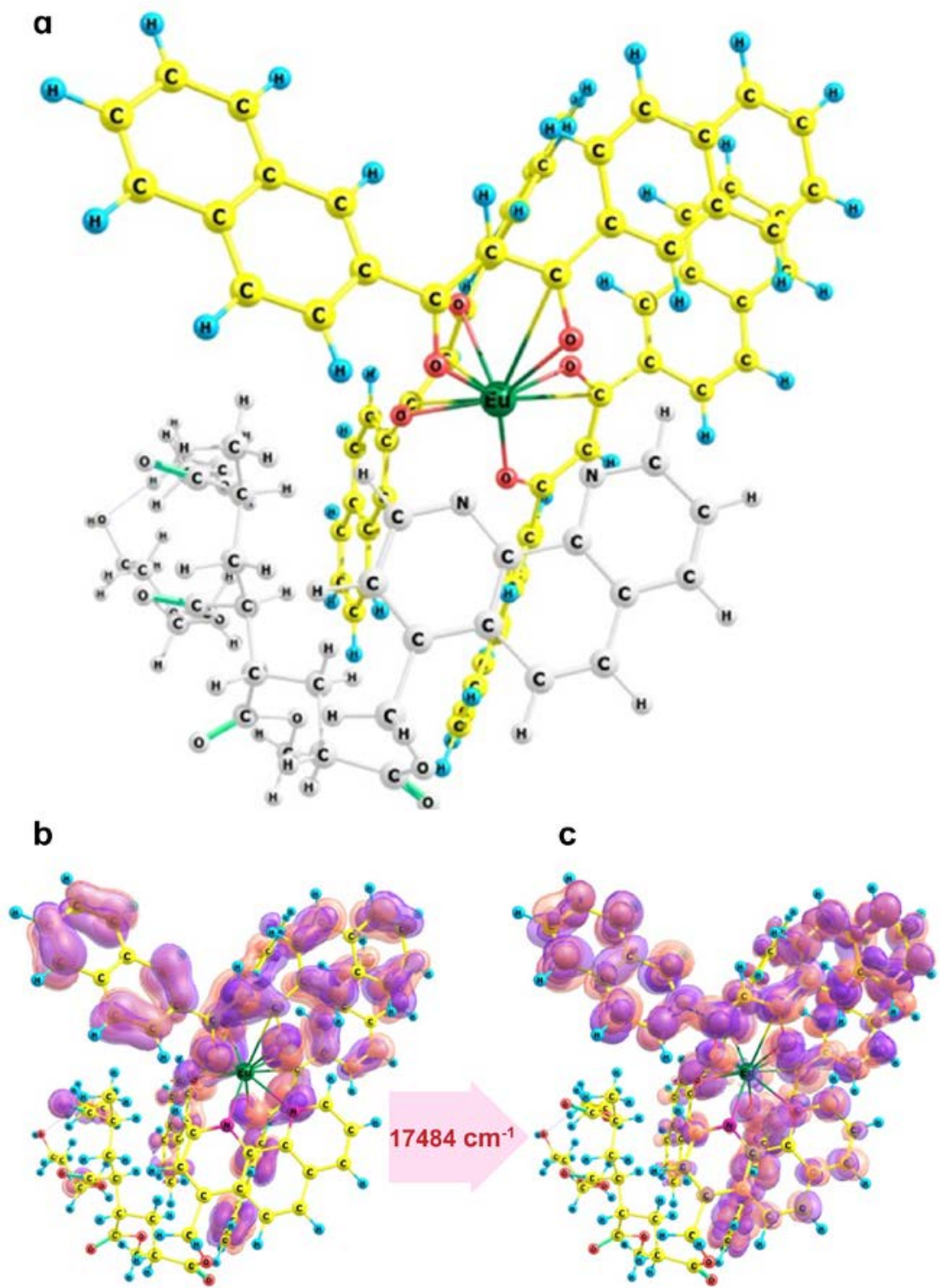

Figure S8. (a) Optimized structure for the DNPD-Ln ${ }^{3+}$ complexes obtained by DFT calculations ( $\omega B$ B97-XD/MWB52 $\left(\mathrm{Eu}^{3+}\right) / \mathrm{MWB} 51\left(\mathrm{Sm}^{3+}\right) / \mathrm{STO}-3 \mathrm{G}$ level of theory). The colorless atoms represent the ancillary ligand (1,10-Phenanthroline + a small polymeric part). (b, c) TD-DFT calculations (in the same level of theory) showing the main molecular orbitals contributions (isosurfaces of $0.03 \boldsymbol{e} / \boldsymbol{a}_{0}{ }^{3}$ ) for the $\mathrm{T}_{1}$ state. It is noticed that there is no significant participation of the ancillary ligand part in the $T_{1}$ state, this state is determined predominantly by DNPD ligands. 


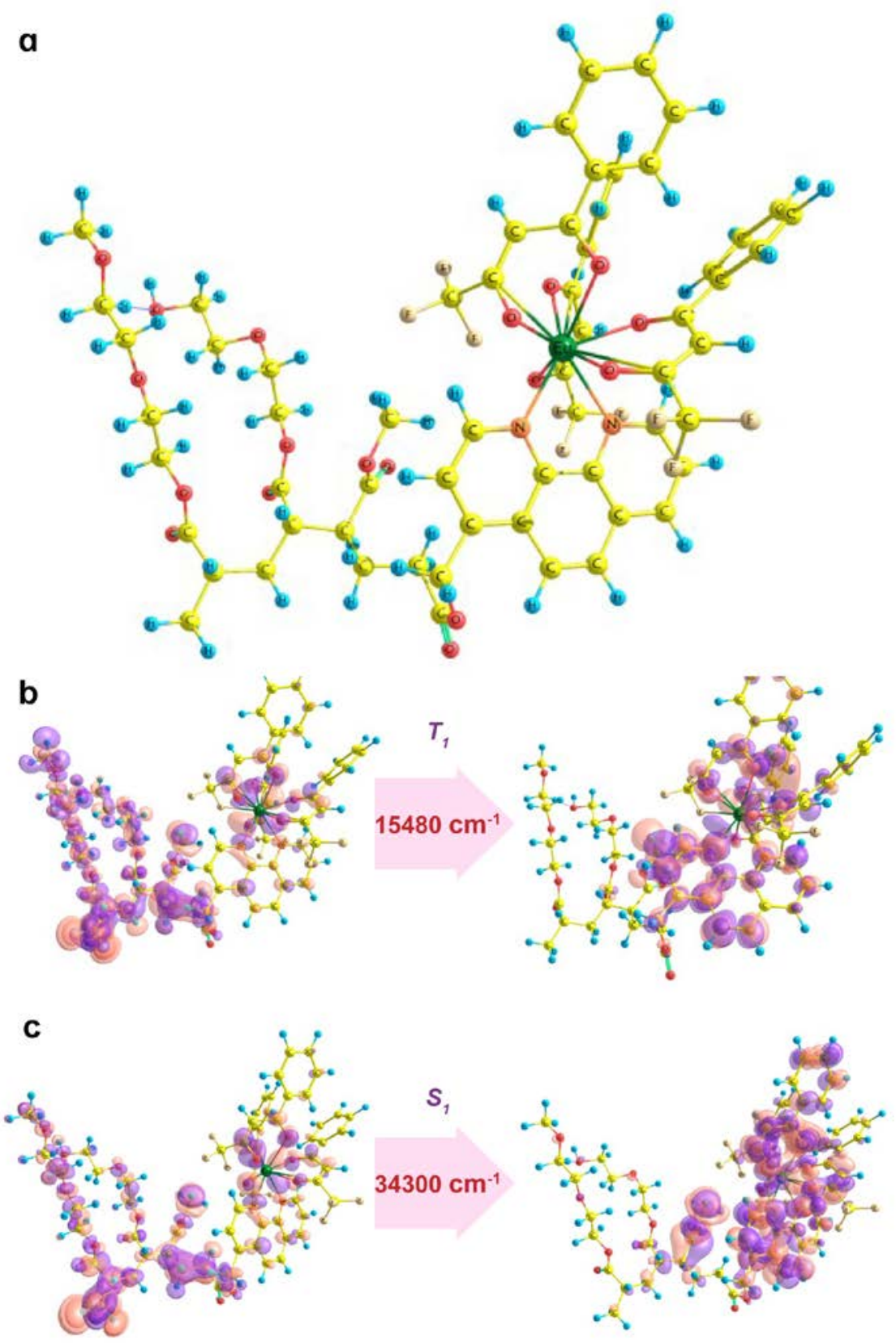

Figure S9. (a) Optimized structure for the btfa- $\mathrm{Ln}^{3+}$ complexes obtained by DFT calculations ( $\omega B$ B7-XD/MWB52( $\left(\mathrm{Eu}^{3+}\right) / \mathrm{MWB} 51\left(\mathrm{Sm}^{3+}\right) / \mathrm{STO}-3 \mathrm{G}$ level of theory). (b, c) TDDFT calculations (in the same level of theory) showing the main molecular orbitals contributions (isosurfaces of $0.03 \boldsymbol{e} / \boldsymbol{a}_{\mathbf{0}}{ }^{3}$ ) for the $\mathrm{T}_{1}$ and $\mathrm{S}_{1}$ states. Both states are formed predominantly by an electronic density flux from the occupied orbitals close to the polymer part to unoccupied orbitals close to the btfa ligands. 


\section{Intramolecular energy transfer}

The intramolecular energy transfer (IET) rates were calculated taking into account the dipole-dipole $\left(W_{d-d}\right)$, dipole-multipole $\left(W_{d-m}\right)$, and exchange mechanisms $\left(W_{e x}\right)$ in coordination compounds, ${ }^{14-17}$ Eqs.S1-S3. The coordination compounds herein analyzed are based on structural data and the excited states obtained from TD-DFT calculations. More details on the quantities in the equations below are in Refs. 14-19.

The IET rates can be obtained by using multipolar and exchange interactions. Eq. S1 stands for the dipole-multipole (4-, 16- and 64-poles) contributions, $K=$ 2,4 , and 6 , respectively.

$$
\begin{gathered}
W_{d-m}=\frac{S_{L}}{(2 J+1) G} \frac{2 \pi e^{2}}{\hbar} \sum_{K}(K+1) \frac{\left\langle r^{K}\right\rangle^{2}}{\left(R_{L}^{K+2}\right)^{2}}\left\langle f\left\|C^{(K)}\right\| f\right\rangle^{2}\left(1-\sigma_{K}\right)^{2} \\
\times\left\langle\psi^{*} J^{*}\left\|U^{(K)}\right\| \psi J\right\rangle^{2} F
\end{gathered}
$$

where $S_{L}$ is the dipole strength of the ligand transition concerning in IET, $\left\langle r^{K}\right\rangle$ are the $4 f$ radial integrals, $G$ is the ligand state degeneracy, $R_{L}$ (equals to 6.6 and 5.9 $\AA$ for the DNPD and btfa-based complexes, respectively) is the donor-acceptor states distance, $\left\langle f\left\|C^{(K)}\right\| f\right\rangle$ is the reduced matrix element of Racah's tensor operator, $\left(1-\sigma_{K}\right)$ are the shielding factor, and $\left\langle\psi^{*} J^{*}\left\|U^{(K)}\right\| \psi J\right\rangle^{2}$ are the squared reduced matrix elements and their values are tabulated in Ref. 20.

For the dipole-dipole contribution, the Eq. S2 is used:

$$
W_{d-d}=\frac{S_{L}\left(1-\sigma_{1}\right)^{2}}{(2 J+1) G} \frac{4 \pi}{\hbar} \frac{e^{2}}{R_{L}^{6}} \sum_{\lambda} \Omega_{K}^{F E D}\left\langle\psi^{*} J^{*}\left\|U^{(K)}\right\| \psi J\right\rangle^{2} F
$$

where the intensity parameters $\Omega_{K}^{F E D}$ are obtained by using only the forced electric dipole mechanism (original Judd-Ofelt theory ${ }^{21,}{ }^{22}$ ) with the Simple Overlap model. ${ }^{23,24}$

The energy transfer rate by the exchange interaction (Eq. S3) can be calculated by:

$$
W_{e x}=\frac{\langle 4 f \mid L\rangle^{4}}{(2 J+1)} \frac{8 \pi}{3 \hbar} \frac{e^{2}}{R_{L}^{4}}\left\langle\psi^{*} J^{*}\|S\| \psi J\right\rangle^{2} \sum_{m}\left|\left\langle\phi\left|\sum_{j} \mu_{z}(j) s_{m}(j)\right| \phi^{*}\right\rangle\right|^{2} F
$$


where $\langle 4 f \mid L\rangle$ is the overlap integral between the ligand donor state and the $4 f$ acceptor state at $R_{L}$ distance, $s_{m}$ the spin operator of electron $j$ in the ligand, $\mu_{z}$ is the dipole operator (z-component), and $\left\langle\psi^{*} J^{*}\|S\| \psi J\right\rangle$ is the reduced matrix elements of the spin operator, which were obtained using free-ion wavefunctions in the intermediate coupling scheme. ${ }^{25}$

The spectral overlap $F$ is related to the energy mismatch condition and contains a sum over Franck-Condon factors. ${ }^{15}$ In the case of energy transfer involving lanthanide complex, $F$ can be obtained by:

$$
F=\frac{1}{\hbar \gamma_{L}} \sqrt{\frac{\ln (2)}{\pi}} e^{-\left(\frac{\Delta}{\hbar \gamma_{L}}\right)^{2} \ln (2)}
$$

The Eq. S4 can be assumed only when the corresponding bandwidth at halfheight for the ligand $\left(\gamma_{L}\right)$ is much larger than the lanthanide $\left(\gamma_{L n}\right)$ ion, $\gamma_{L} \gg \gamma_{L n} \cdot{ }^{26}$ The $\Delta$ (in $\mathrm{cm}^{-1}$ ) is the band maximum energy difference between ligand donor state and lanthanide ion acceptor state, $\Delta E=E_{L}-E_{L n}$. The forward energy transfer rates $\left(W_{E T}\right)$ can be calculated by the sum over all Eq. S1, Eq. S2 and Eq. S3 in the same pathway:

$$
W_{E T}=W_{d-m}+W_{d-d}+W_{e x}
$$

The backward energy transfer rates $\left(W_{b E T}\right)$, that is, the energy returned from acceptor to donor state, is obtained with the same above equations, except for multiplying the spectral overlap $F$ (Eq. S4) by the Boltzmann's factor:

$$
F_{b E T}=\frac{1}{\hbar \gamma_{L}} \sqrt{\frac{\ln (2)}{\pi}} e^{-\left(\frac{\Delta \mathrm{E}}{\hbar \gamma_{L}}\right)^{2} \ln (2)} \times e^{-\left(\frac{|\Delta \mathrm{E}|}{k_{B} T}\right)}
$$

where $k_{B}$ is the Boltzmann's constant. 


\section{Energy transfer pathways}

The pathways and its predominant mechanism to the forward intramolecular energy transfer rates ( $\left.\mathrm{W}_{\mathrm{ET}}\right)$ for the complexes inside the polymeric micelle structure are shown in Table S2 and Table S3 for DNPD-based polymeric micelles and Table S5 and Table S6 for btfa-based micelles. It was analyzed different energy transfer pathways in which ligand-to-metal energy transfer can occur, from both $\mathrm{T}_{1}\left(17500 \mathrm{~cm}^{-1}\right.$ and $\left.15480 \mathrm{~cm}^{-1}\right)$ or $\mathrm{S}_{1}\left(30000 \mathrm{~cm}^{-1}\right.$ and 34300 $\mathrm{cm}^{-1}$ ) ligand states for DNPD and BTFA, respectively.

Table S2. Intramolecular energy transfer rates (in $\mathrm{s}^{-1}$ ) for $E u^{3+}$-DNPD complex in polymeric micelles. The donor-acceptor distance is $R_{\mathrm{L}}=6.6 \AA$.

\begin{tabular}{|c|c|c|c|c|}
\hline Pathway & Donor State & Acceptor State & $W_{E T}$ & Mechanism \\
\hline 1 & $T_{1}$ & ${ }^{7} \mathrm{~F}_{1} \rightarrow{ }^{5} \mathrm{D}_{0}$ & $1.972 \times 10^{6}$ & exchange \\
\hline 2 & $T_{1}$ & ${ }^{7} \mathrm{~F}_{0} \rightarrow{ }^{5} \mathrm{D}_{0}$ & 0.447 & dipole-multipole* \\
\hline 3 & $T_{1}$ & ${ }^{7} F_{1} \rightarrow{ }^{5} D_{1}$ & 1.734 & exchange \\
\hline 4 & $T_{1}$ & ${ }^{7} \mathrm{~F}_{0} \rightarrow{ }^{5} \mathrm{D}_{1}$ & $1.504 \times 10^{3}$ & exchange \\
\hline 5 & $T_{1}$ & ${ }^{7} \mathrm{~F}_{1} \rightarrow{ }^{5} \mathrm{D}_{2}$ & $1.236 \times 10^{-3}$ & exchange \\
\hline 6 & $T_{1}$ & ${ }^{7} \mathrm{~F}_{0} \rightarrow{ }^{5} \mathrm{D}_{2}$ & $4.191 \times 10^{-7}$ & dipole-multipole \\
\hline 7 & $S_{1}$ & ${ }^{7} \mathrm{~F}_{1} \rightarrow{ }^{5} \mathrm{D}_{0}$ & $1.898 \times 10^{3}$ & exchange \\
\hline 8 & $S_{1}$ & ${ }^{7} \mathrm{~F}_{0} \rightarrow{ }^{5} \mathrm{D}_{0}$ & 12.39 & dipole-multipole* \\
\hline 9 & $S_{1}$ & ${ }^{7} \mathrm{~F}_{1} \rightarrow{ }^{5} \mathrm{D}_{1}$ & $2.857 \times 10^{3}$ & dipole-multipole \\
\hline 10 & $S_{1}$ & ${ }^{7} \mathrm{~F}_{0} \rightarrow{ }^{5} \mathrm{D}_{1}$ & $2.319 \times 10^{4}$ & exchange \\
\hline 11 & $S_{1}$ & ${ }^{7} \mathrm{~F}_{1} \rightarrow{ }^{5} \mathrm{D}_{2}$ & $4.109 \times 10^{3}$ & exchange \\
\hline 12 & $S_{1}$ & ${ }^{7} \mathrm{~F}_{0} \rightarrow{ }^{5} \mathrm{D}_{2}$ & $2.313 \times 10^{5}$ & dipole-multipole \\
\hline 13 & $S_{1}$ & ${ }^{7} F_{0} \rightarrow{ }^{5} D_{3}$ & 0.000 & none \\
\hline 14 & $S_{1}$ & ${ }^{7} \mathrm{~F}_{1} \rightarrow{ }^{5} \mathrm{D}_{3}$ & $1.108 \times 10^{5}$ & dipole-multipole \\
\hline 15 & $S_{1}$ & ${ }^{7} \mathrm{~F}_{0} \rightarrow{ }^{5} \mathrm{~L}_{6}$ & $3.170 \times 10^{4}$ & dipole-dipole \\
\hline 16 & $S_{1}$ & ${ }^{7} \mathrm{~F}_{1} \rightarrow{ }^{5} \mathrm{~L}_{6}$ & $2.773 \times 10^{3}$ & dipole-dipole \\
\hline \multirow[t]{2}{*}{17} & $S_{1}$ & ${ }^{7} \mathrm{~F}_{0} \rightarrow{ }^{5} \mathrm{D}_{4}$ & $1.368 \times 10^{3}$ & dipole-dipole \\
\hline & & Total & $2.383 \times 10^{6}$ & \\
\hline
\end{tabular}

${ }^{*}$ Results obtained considering the J-mixing effect. 
Table S3. Intramolecular energy transfer rates (in $\mathrm{s}^{-1}$ ) for $\mathrm{Sm}^{3+}$-DNPD complex in polymeric micelles. The donor-acceptor distance is $R_{\mathrm{L}}=6.6 \AA$.

\begin{tabular}{|c|c|c|c|c|}
\hline Pathway & Donor State & Acceptor State & $\mathbf{W}_{\mathrm{ET}}$ & Mechanism \\
\hline 1 & $T_{1}$ & ${ }^{6} \mathrm{H}_{5 / 2} \rightarrow{ }^{4} \mathrm{G}_{5 / 2}$ & $3.053 \times 10^{5}$ & exchange \\
\hline 2 & $T_{1}$ & ${ }^{6} \mathrm{H}_{5 / 2} \rightarrow{ }^{4} \mathrm{~F}_{3 / 2}$ & 0.000 & none \\
\hline 3 & $T_{1}$ & ${ }^{6} \mathrm{H}_{5 / 2} \rightarrow{ }^{4} \mathrm{G}_{7 / 2}$ & $5.059 \times 10^{-2}$ & exchange \\
\hline 4 & $T_{1}$ & ${ }^{6} \mathrm{H}_{5 / 2} \rightarrow{ }^{4} \mathrm{I}_{9 / 2}$ & $9.471 \times 10^{-6}$ & dipole-multipole \\
\hline 5 & $T_{1}$ & ${ }^{6} \mathrm{H}_{5 / 2} \rightarrow{ }^{4} \mathrm{M}_{15 / 2}$ & $2.641 \times 10^{-7}$ & dipole-dipole \\
\hline 6 & $T_{1}$ & ${ }^{6} \mathrm{H}_{5 / 2} \rightarrow{ }^{4} \mathrm{I}_{11 / 2}$ & $1.785 \times 10^{-8}$ & dipole-dipole \\
\hline 7 & $T_{1}$ & ${ }^{6} \mathrm{H}_{5 / 2} \rightarrow{ }^{4} \mathrm{I}_{13 / 2}$ & $3.286 \times 10^{-9}$ & dipole-dipole \\
\hline 8 & $T_{1}$ & ${ }^{6} \mathrm{H}_{5 / 2} \rightarrow{ }^{4} \mathrm{~F}_{5 / 2}$ & $1.246 \times 10^{-6}$ & exchange \\
\hline 9 & $T_{1}$ & ${ }^{6} \mathrm{H}_{5 / 2} \rightarrow{ }^{4} \mathrm{M}_{17 / 2}$ & $4.463 \times 10^{-12}$ & dipole-dipole \\
\hline 10 & $T_{1}$ & ${ }^{6} \mathrm{H}_{5 / 2} \rightarrow{ }^{4} \mathrm{G}_{9 / 2}$ & $7.126 \times 10^{-13}$ & dipole-dipole \\
\hline 11 & $S_{1}$ & ${ }^{6} \mathrm{H}_{5 / 2} \rightarrow{ }^{4} \mathrm{G}_{5 / 2}$ & 0.687 & exchange \\
\hline 12 & $S_{1}$ & ${ }^{6} \mathrm{H}_{5 / 2} \rightarrow{ }^{4} \mathrm{~F}_{3 / 2}$ & 0.000 & None \\
\hline 13 & $S_{1}$ & ${ }^{6} \mathrm{H}_{5 / 2} \rightarrow{ }^{4} \mathrm{G}_{7 / 2}$ & $1.308 \times 10^{3}$ & dipole-multipole \\
\hline 14 & $S_{1}$ & ${ }^{6} \mathrm{H}_{5 / 2} \rightarrow{ }^{4} I_{9 / 2}$ & $1.837 \times 10^{4}$ & dipole-multipole \\
\hline 15 & $S_{1}$ & ${ }^{6} \mathrm{H}_{5 / 2} \rightarrow{ }^{4} \mathrm{M}_{15 / 2}$ & $1.530 \times 10^{3}$ & dipole-dipole \\
\hline 16 & $S_{1}$ & ${ }^{6} \mathrm{H}_{5 / 2} \rightarrow{ }^{4} \mathrm{I}_{11 / 2}$ & $5.114 \times 10^{2}$ & dipole-dipole \\
\hline 17 & $S_{1}$ & ${ }^{6} \mathrm{H}_{5 / 2} \rightarrow{ }^{4} \mathrm{I}_{13 / 2}$ & $3.668 \times 10^{3}$ & dipole-dipole \\
\hline 18 & $S_{1}$ & ${ }^{6} \mathrm{H}_{5 / 2} \rightarrow{ }^{4} \mathrm{~F}_{5 / 2}$ & $3.747 \times 10^{2}$ & exchange \\
\hline 19 & $S_{1}$ & ${ }^{6} \mathrm{H}_{5 / 2} \rightarrow{ }^{4} \mathrm{M}_{17 / 2}$ & $2.643 \times 10^{3}$ & dipole-dipole \\
\hline 20 & $S_{1}$ & ${ }^{6} \mathrm{H}_{5 / 2} \rightarrow{ }^{4} \mathrm{G}_{9 / 2}$ & $2.332 \times 10^{3}$ & dipole-dipole \\
\hline
\end{tabular}

Pathway 1, for both $\mathrm{Eu}^{3+}$ and $\mathrm{Sm}^{3+}$ as acceptors, is the dominant one and corresponds to $\sim 83 \%$ and $\sim 91 \%$ of the total forward IET rates, respectively. The backward energies transfer rates $\left(W_{b E T}\right)$ from pathways 1 are the keys to explain why the intensities decrease when the temperature grows. Indeed, Figure S10. shows the increase in $W_{b E T}$ when the temperature increases, depopulating the emitting levels ${ }^{5} \mathrm{D}_{0}\left(\mathrm{Eu}^{3+}\right)$ and ${ }^{4} \mathrm{G}_{5 / 2}\left(\mathrm{Sm}^{3+}\right) . W_{b E T}$ for Eu-DNPD is more affected by the temperature than the Sm-DNPD one, explaining why the integrated area (Figure $3 b$ ) of ${ }^{5} \mathrm{D}_{0} \rightarrow{ }^{7} \mathrm{~F}_{2}$ transition decay faster than the integrated area of ${ }^{4} \mathrm{G}_{5 / 2}$ $\rightarrow{ }^{6} \mathrm{H}_{9 / 2}$ one, as pointed in Figure S10. 


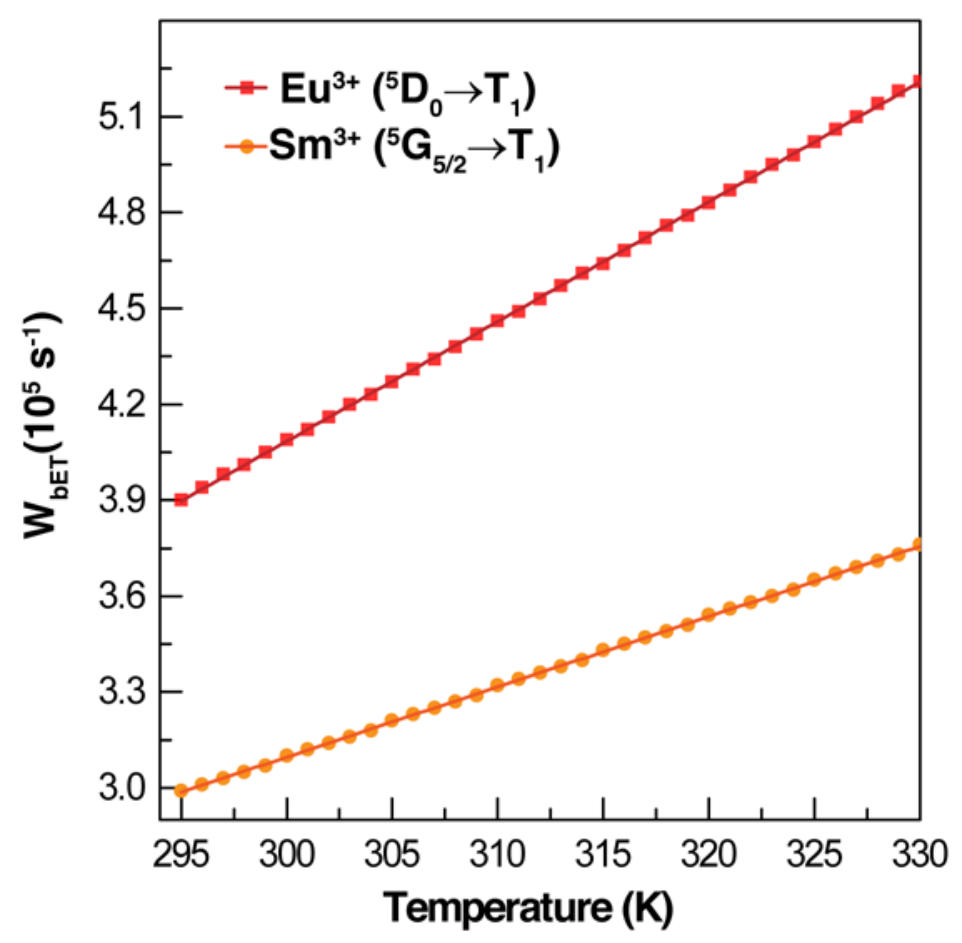

Figure S10. Backward IET rates from the emitters levels of $\mathrm{Eu}^{3+}\left({ }^{5} \mathrm{D}_{0}\right)$ and $\mathrm{Sm}^{3+}\left({ }^{4} \mathrm{G}_{5 / 2}\right)$ ions for DNPD-based lanthanide complexes. The rate ${ }^{5} D_{0} \rightarrow T_{1}$ grows faster than the ${ }^{4} \mathrm{G}_{5 / 2} \rightarrow \mathrm{T}_{1}$, resulting in a more effective depopulation on ${ }^{5} \mathrm{D}_{0}$ level, therefore, the integrated area of the ${ }^{5} D_{0} \rightarrow{ }^{7} F_{2}$ transition decreases faster than the integrated area of the ${ }^{5} \mathrm{G}_{5 / 2} \rightarrow{ }^{6} \mathrm{H}_{9 / 2}$. The dependences were fitted by straight lines (Table S4).

Table S4. Linear coefficients and their respective uncertainties for the backward IET rates tendencies of Figure S10.

\begin{tabular}{cccc}
\hline Ion & Intercept $\left(\mathbf{s}^{-1}\right)$ & Slope $\left(10^{-2} \mathbf{s}^{-1} \cdot \mathbf{K}^{-1}\right)$ & $r^{2}$ \\
\hline $\mathrm{Eu}^{3+}$ & $-7.15 \pm 0.02$ & $3.746 \pm 0.007$ & 0.99 \\
$\mathrm{Sm}^{3+}$ & $-3.50 \pm 0.02$ & $2.198 \pm 0.005$ & 0.99 \\
\hline
\end{tabular}


Table S5. Intramolecular energy transfer rates (in s $\mathrm{s}^{-1}$ ) for $\mathrm{Eu}^{3+}-\mathrm{btfa}$ complex in polymeric micelles. The donor-acceptor distance is $R_{\mathrm{L}}=5.9 \AA \hat{\text {. }}$.

\begin{tabular}{|c|c|c|c|c|}
\hline Pathway & Donor State & Acceptor State & $\mathbf{W}_{\mathrm{ET}}$ & Mechanism \\
\hline 1 & $T_{1}$ & ${ }^{7} \mathrm{~F}_{1} \rightarrow{ }^{5} \mathrm{D}_{0}$ & $6.453 \times 10^{3}$ & exchange \\
\hline 2 & $T_{1}$ & ${ }^{7} F_{0} \rightarrow{ }^{5} D_{0}$ & $1.671 \times 10^{-4}$ & dipole-multipole* \\
\hline 3 & $T_{1}$ & ${ }^{7} F_{1} \rightarrow{ }^{5} D_{1}$ & $2.788 \times 10^{-4}$ & exchange \\
\hline 4 & $T_{1}$ & ${ }^{7} F_{0} \rightarrow{ }^{5} D_{1}$ & 0.229 & exchange \\
\hline 5 & $T_{1}$ & ${ }^{7} F_{1} \rightarrow{ }^{5} D_{2}$ & $1.316 \times 10^{-7}$ & exchange \\
\hline 6 & $T_{1}$ & ${ }^{7} F_{0} \rightarrow{ }^{5} D_{2}$ & $3.035 \times 10^{-11}$ & dipole-multipole \\
\hline 7 & $S_{1}$ & ${ }^{7} F_{1} \rightarrow{ }^{5} D_{0}$ & 22.77 & exchange \\
\hline 8 & $S_{1}$ & ${ }^{7} \mathrm{~F}_{0} \rightarrow{ }^{5} \mathrm{D}_{0}$ & 0.108 & dipole-multipole \\
\hline 9 & $S_{1}$ & ${ }^{7} F_{1} \rightarrow{ }^{5} D_{1}$ & 42.48 & dipole-multipole \\
\hline 10 & $S_{1}$ & ${ }^{7} \mathrm{~F}_{0} \rightarrow{ }^{5} \mathrm{D}_{1}$ & $6.084 \times 10^{2}$ & exchange \\
\hline 11 & $S_{1}$ & ${ }^{7} F_{1} \rightarrow{ }^{5} D_{2}$ & $2.339 \times 10^{2}$ & exchange \\
\hline 12 & $S_{1}$ & ${ }^{7} F_{0} \rightarrow{ }^{5} D_{2}$ & $1.014 \times 10^{4}$ & dipole-multipole \\
\hline 13 & $S_{1}$ & ${ }^{7} F_{0} \rightarrow{ }^{5} D_{3}$ & 0.000 & none \\
\hline 14 & $S_{1}$ & ${ }^{7} F_{1} \rightarrow{ }^{5} D_{3}$ & $2.739 \times 10^{3}$ & dipole-multipole \\
\hline 15 & $S_{1}$ & ${ }^{7} \mathrm{~F}_{0} \rightarrow{ }^{5} \mathrm{~L}_{6}$ & $4.839 \times 10^{3}$ & dipole-dipole \\
\hline 16 & $S_{1}$ & ${ }^{7} \mathrm{~F}_{1} \rightarrow{ }^{5} \mathrm{~L}_{6}$ & $3.680 \times 10^{2}$ & dipole-dipole \\
\hline 17 & $S_{1}$ & ${ }^{7} \mathrm{~F}_{0} \rightarrow{ }^{5} \mathrm{D}_{4}$ & $1.714 \times 10^{3}$ & dipole-dipole \\
\hline 18 & $S_{1}$ & ${ }^{7} F_{1} \rightarrow{ }^{5} G_{2}$ & $6.128 \times 10^{4}$ & exchange \\
\hline 19 & $S_{1}$ & ${ }^{7} F_{1} \rightarrow{ }^{5} G_{3}$ & $8.721 \times 10^{3}$ & dipole-multipole \\
\hline & & Total & $9.716 \times 10^{4}$ & \\
\hline
\end{tabular}

* Results obtained considering the J-mixing effect. 
Table S6. Intramolecular energy transfer rates (in $\mathrm{s}^{-1}$ ) for $\mathrm{Sm}^{3+}$-btfa complex in polymeric micelles. The donor-acceptor distance is $R_{\mathrm{L}}=5.9 \AA$.

\begin{tabular}{|c|c|c|c|c|}
\hline Pathway & Donor State & Acceptor State & $\mathbf{W}_{\mathrm{ET}}$ & Mechanism \\
\hline 1 & $T_{1}$ & ${ }^{6} \mathrm{H}_{5 / 2} \rightarrow{ }^{4} \mathrm{G}_{5 / 2}$ & $2.559 \times 10^{5}$ & exchange \\
\hline 2 & $T_{1}$ & ${ }^{6} \mathrm{H}_{5 / 2} \rightarrow{ }^{4} \mathrm{~F}_{3 / 2}$ & 0.000 & none \\
\hline 3 & $T_{1}$ & ${ }^{6} \mathrm{H}_{5 / 2} \rightarrow{ }^{4} \mathrm{G}_{7 / 2}$ & $6.456 \times 10^{-2}$ & exchange \\
\hline 4 & $T_{1}$ & ${ }^{6} \mathrm{H}_{5 / 2} \rightarrow{ }^{4} \mathrm{I}_{9 / 2}$ & $8.037 \times 10^{-10}$ & dipole-multipole \\
\hline 5 & $T_{1}$ & ${ }^{6} \mathrm{H}_{5 / 2} \rightarrow{ }^{4} \mathrm{M}_{15 / 2}$ & $1.726 \times 10^{-11}$ & dipole-dipole \\
\hline 6 & $T_{1}$ & ${ }^{6} \mathrm{H}_{5 / 2} \rightarrow{ }^{4} \mathrm{I}_{11 / 2}$ & $1.104 \times 10^{-12}$ & dipole-dipole \\
\hline 7 & $T_{1}$ & ${ }^{6} \mathrm{H}_{5 / 2} \rightarrow{ }^{4} \mathrm{I}_{13 / 2}$ & $1.904 \times 10^{-13}$ & dipole-dipole \\
\hline 8 & $T_{1}$ & ${ }^{6} \mathrm{H}_{5 / 2} \rightarrow{ }^{4} \mathrm{~F}_{5 / 2}$ & $1.097 \times 10^{-6}$ & exchange \\
\hline 9 & $T_{1}$ & ${ }^{6} \mathrm{H}_{5 / 2} \rightarrow{ }^{4} \mathrm{M}_{17 / 2}$ & $2.140 \times 10^{-16}$ & dipole-dipole \\
\hline 10 & $T_{1}$ & ${ }^{6} \mathrm{H}_{5 / 2} \rightarrow{ }^{4} \mathrm{G}_{9 / 2}$ & $3.420 \times 10^{-17}$ & dipole-dipole \\
\hline 11 & $S_{1}$ & ${ }^{6} \mathrm{H}_{5 / 2} \rightarrow{ }^{4} \mathrm{G}_{5 / 2}$ & $1.999 \times 10^{4}$ & exchange \\
\hline 12 & $S_{1}$ & ${ }^{6} \mathrm{H}_{5 / 2} \rightarrow{ }^{4} \mathrm{~F}_{3 / 2}$ & 0.000 & none \\
\hline 13 & $S_{1}$ & ${ }^{6} \mathrm{H}_{5 / 2} \rightarrow{ }^{4} \mathrm{G}_{7 / 2}$ & $2.350 \times 10^{5}$ & exchange \\
\hline 14 & $S_{1}$ & ${ }^{6} \mathrm{H}_{5 / 2} \rightarrow{ }^{4} \mathrm{I}_{9 / 2}$ & $6.433 \times 10^{2}$ & dipole-multipole \\
\hline 15 & $S_{1}$ & ${ }^{6} \mathrm{H}_{5 / 2} \rightarrow{ }^{4} \mathrm{M}_{15 / 2}$ & 46.82 & dipole-dipole \\
\hline 16 & $S_{1}$ & ${ }^{6} \mathrm{H}_{5 / 2} \rightarrow{ }^{4} \mathrm{I}_{11 / 2}$ & 14.70 & dipole-dipole \\
\hline 17 & $S_{1}$ & ${ }^{6} \mathrm{H}_{5 / 2} \rightarrow{ }^{4} \mathrm{I}_{13 / 2}$ & 97.03 & dipole-dipole \\
\hline 18 & $S_{1}$ & ${ }^{6} \mathrm{H}_{5 / 2} \rightarrow{ }^{4} \mathrm{~F}_{5 / 2}$ & $3.637 \times 10^{6}$ & exchange \\
\hline 19 & $S_{1}$ & ${ }^{6} \mathrm{H}_{5 / 2} \rightarrow{ }^{4} \mathrm{M}_{17 / 2}$ & 57.44 & dipole-dipole \\
\hline \multirow[t]{2}{*}{20} & $S_{1}$ & ${ }^{6} \mathrm{H}_{5 / 2} \rightarrow{ }^{4} \mathrm{G}_{9 / 2}$ & $4.536 \times 10^{3}$ & dipole-dipole \\
\hline & & Total & $4.153 \times 10^{6}$ & \\
\hline
\end{tabular}




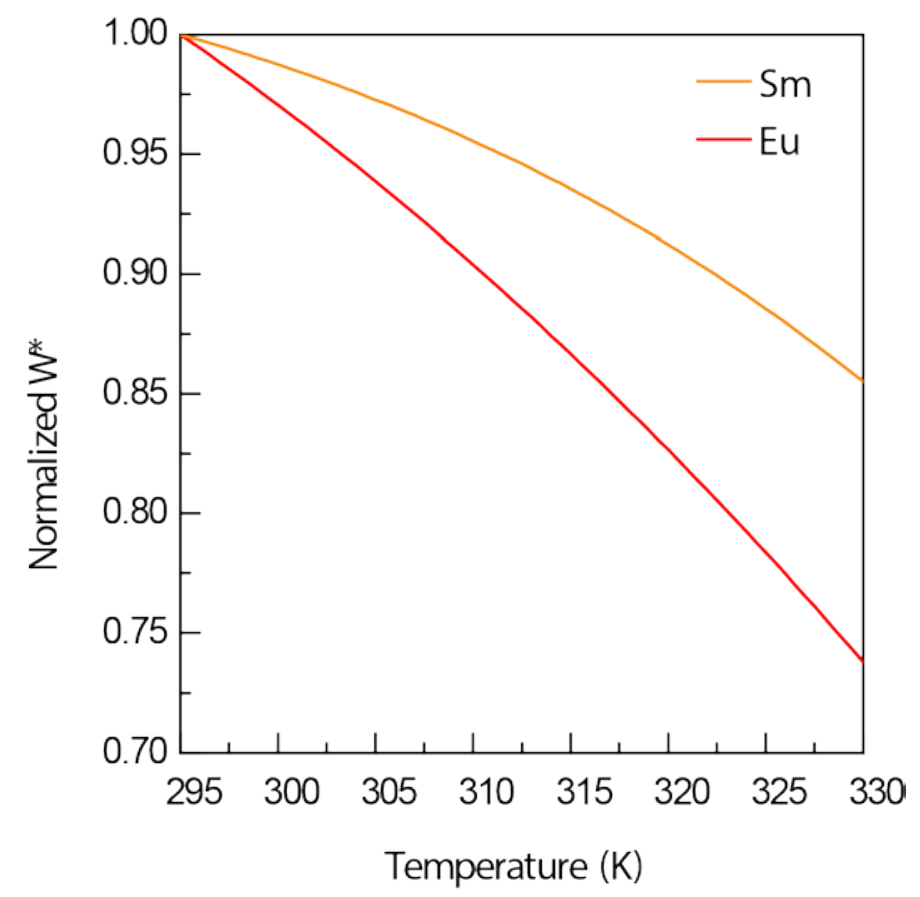

Figure S11. Temperature dependence of the normalized $\mathrm{W}^{*}$ for btfa-based lanthanide complexes. The energy transfer from the $\mathrm{Eu}^{3+}$ emitting level to the $\mathrm{T}_{1}$ state is in the same order of magnitude of the energy transfer from $\mathrm{S}_{1}$ to the $\mathrm{Eu}^{3+}$ higher states. In the case of $\mathrm{Sm}^{3+}$, the $\mathbf{W}_{\mathbf{T}_{\mathbf{1}}}^{\mathbf{L n}}$ is one order of magnitude lower than the $\mathbf{W}_{\mathbf{L n}}^{\mathbf{S}}$. This corroborates with the temperature dependence of the integrated areas as shown in Figure $3 a$. 


\section{Photoluminescence}

\section{Room temperature excitation}
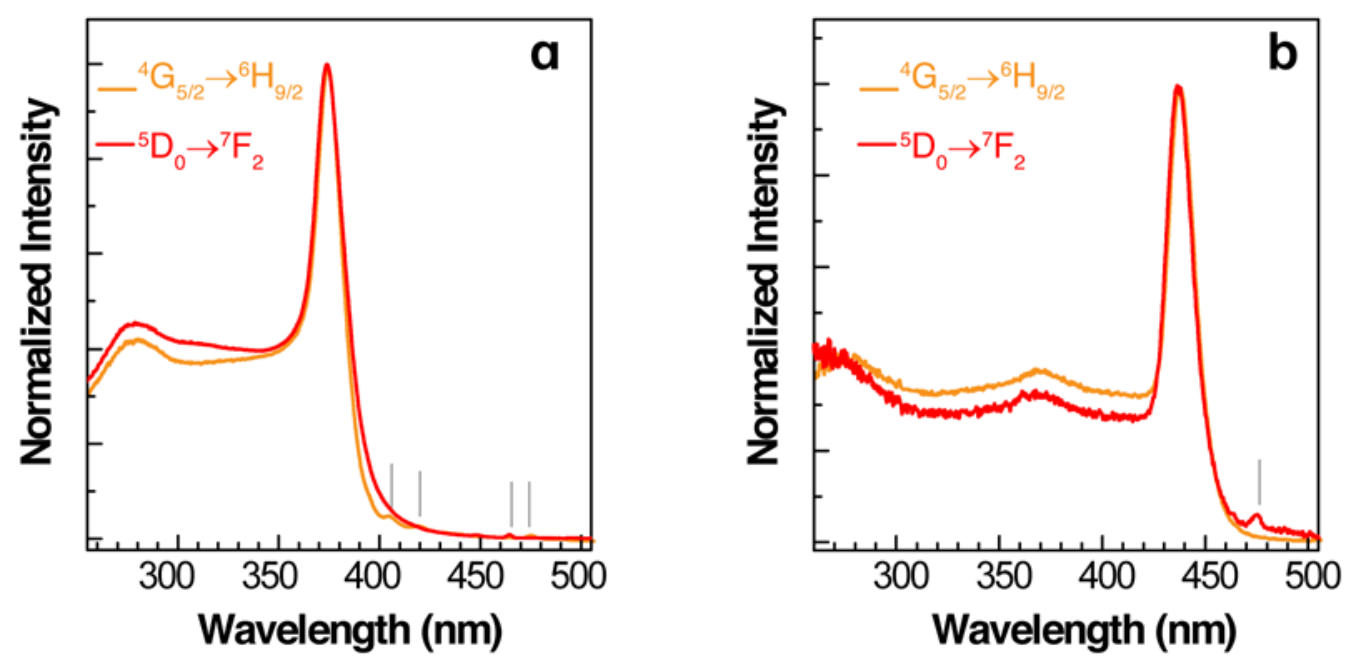

Figure S12. Room-temperature excitation spectra monitoring ${ }^{5} \mathrm{D}_{0} \rightarrow{ }^{7} \mathrm{~F}_{2}$ transition of Eu $\mathrm{u}^{3+}$ and ${ }^{4} \mathrm{G}_{5 / 2} \rightarrow{ }^{6} \mathrm{H}_{9 / 2}$ transition of $\mathrm{Sm}^{3+}$ in (a) btfa and (b) DNPD-based micelles. The corresponding absorption spectra are presented in (c) and (d), respectively.

Regardless of the monitored emission line of $\mathrm{Eu}^{3+}\left(\mathrm{or} \mathrm{Sm}^{3+}\right)$ in both polymeric micelles at broadband is observed in excitation spectra with a maximum around $380 \mathrm{~nm}$ for btfa-based polymeric micelles and $440 \mathrm{~nm}$ for DNPD-based material. These absorption bands are ascribed to the ligand absorption.

The emission spectra in the physiological temperature range were recorded for thermometric analysis. Firstly, a straight baseline was removed for each spectrum and the conversion from wavelength to energy scale was done. Also the intensity as a function of wavelength, $f(\lambda)$, in the wavelength range $d \lambda$, was converted to intensity as a function of energy, $f(E)$, in the energy range $d E$, using a Jacobian transformation: ${ }^{27}$

$$
f(E)=f(\lambda) \frac{d I_{\lambda}}{d E} \frac{d \lambda}{d E}=-f\left(I_{\lambda}\right) \frac{h c}{E^{2}}
$$

where $h$ and $c$ denote the Plank constant and the speed of light in vacuum, respectively. 
The integrated areas $\left(I_{i}\right)$ of the indicated transitions were calculated after removed the baseline and used the Jacobian transformation. The error in the integrated areas is estimated multiplying the standard deviation of noise by the integrated area, for each spectrum.

\section{Thermometric performance of the $\mathrm{Ln}^{3+}$-containing micelles}

Table S7. Parameters resulting from the fitting of a polynomial function $\Delta=A+B T+C T^{2}$ in the btfa- and DNPD-based polymeric micelles.

\begin{tabular}{lllll}
\hline Ligand & $\mathbf{A}$ & $\mathbf{B}\left(\mathbf{1 0}^{-3} \mathbf{K}^{-\mathbf{1}}\right)$ & $\mathbf{C ~}\left(\mathbf{1 0}^{-\mathbf{5}} \mathbf{K}^{-\mathbf{2}}\right)$ & $\boldsymbol{r}^{\mathbf{2}}$ \\
\hline$b t f a$ & $1.31 \pm 0.46$ & $-8.96 \pm 0.30$ & $1.59 \pm 0.47$ & 0.988 \\
DNPD & $9.76 \pm 0.4$ & $-6.51 \pm 0.26$ & $11.38 \pm 0.42$ & 0.982 \\
\hline
\end{tabular}
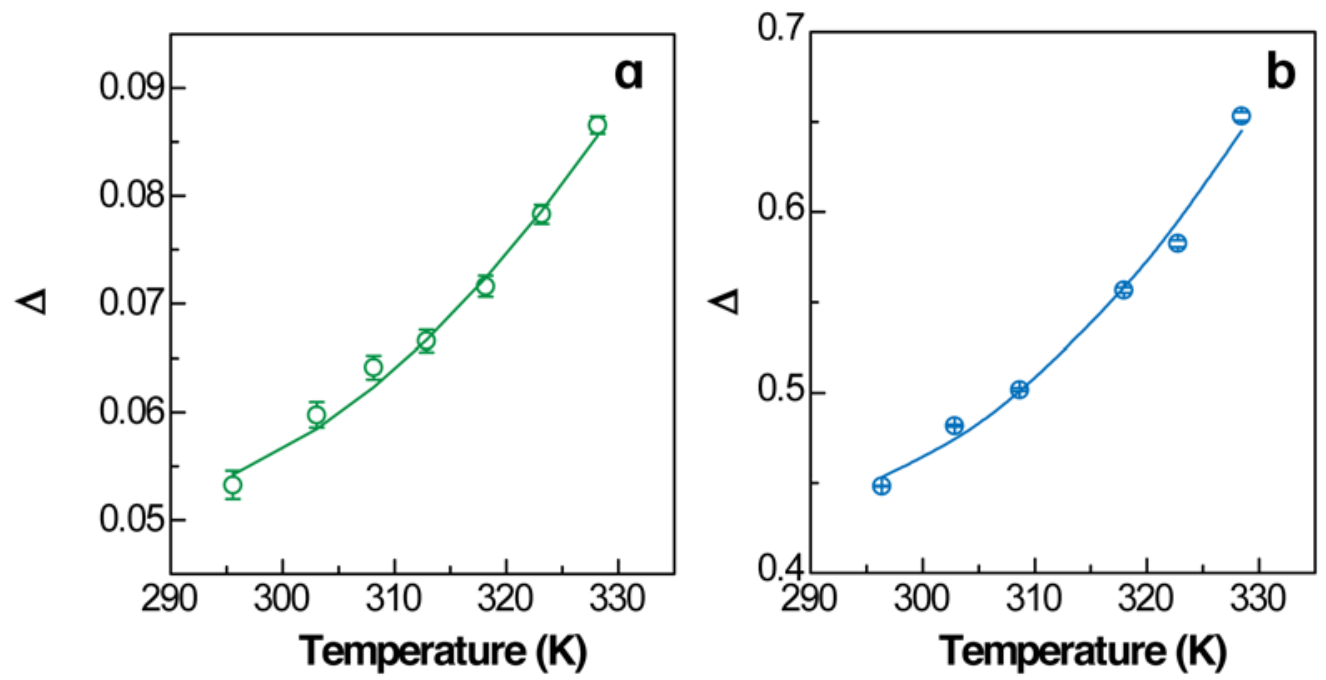

Figure S13. Calibration curve of (a) btfa- and (b) DNPD-based polymeric micelles. The lines are the fits to empirical functions with parameters presented in Table S7.

The relative thermal sensitivity $\left(S_{r}\right)$ was calculated using:

$$
S_{r}=\frac{1}{\Delta}\left|\frac{\partial \Delta}{\partial T}\right|
$$

and the temperature uncertainty, $\delta T$ :

$$
\delta T=\frac{\sqrt{2}}{S_{r}} \frac{\delta I(T)}{I(T)}
$$


where $\delta l / l$ is a function of temperature. ${ }^{28}$
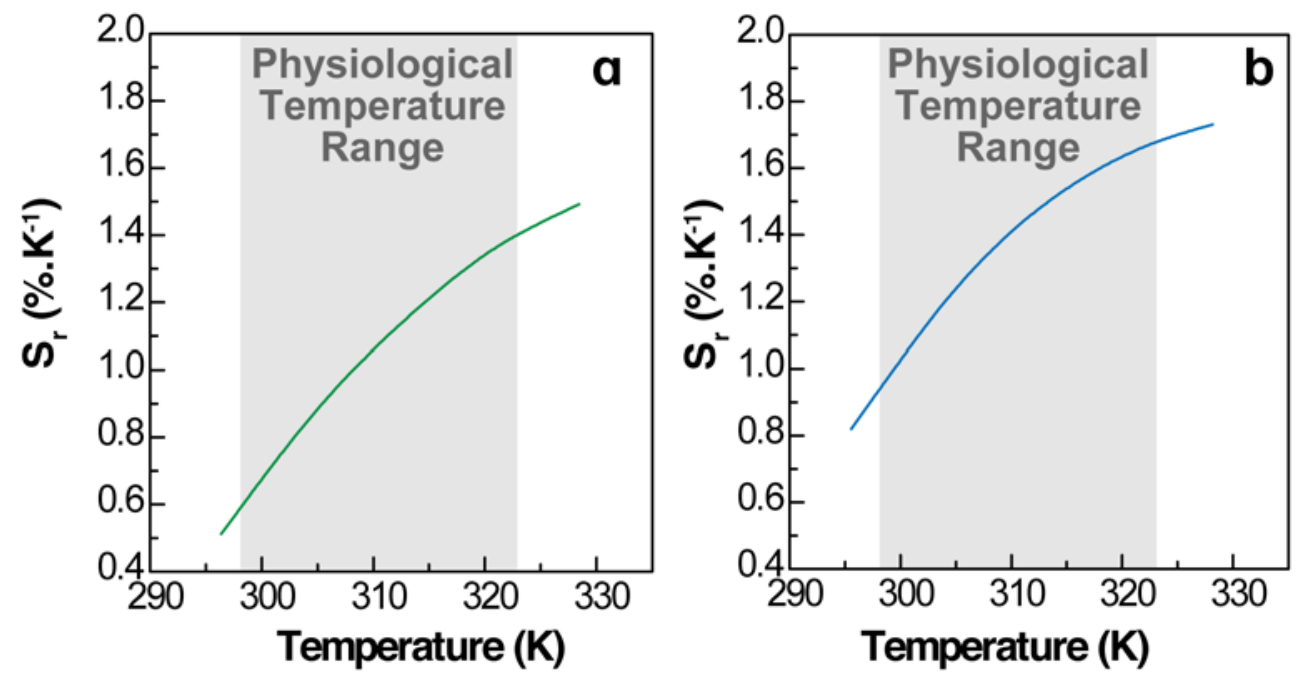

Figure S14. Relative thermal sensitivity, $S_{r}$, of (a) btfa- and (b) DNPD-based polymeric micelles. The physiological temperature range is shadowed.
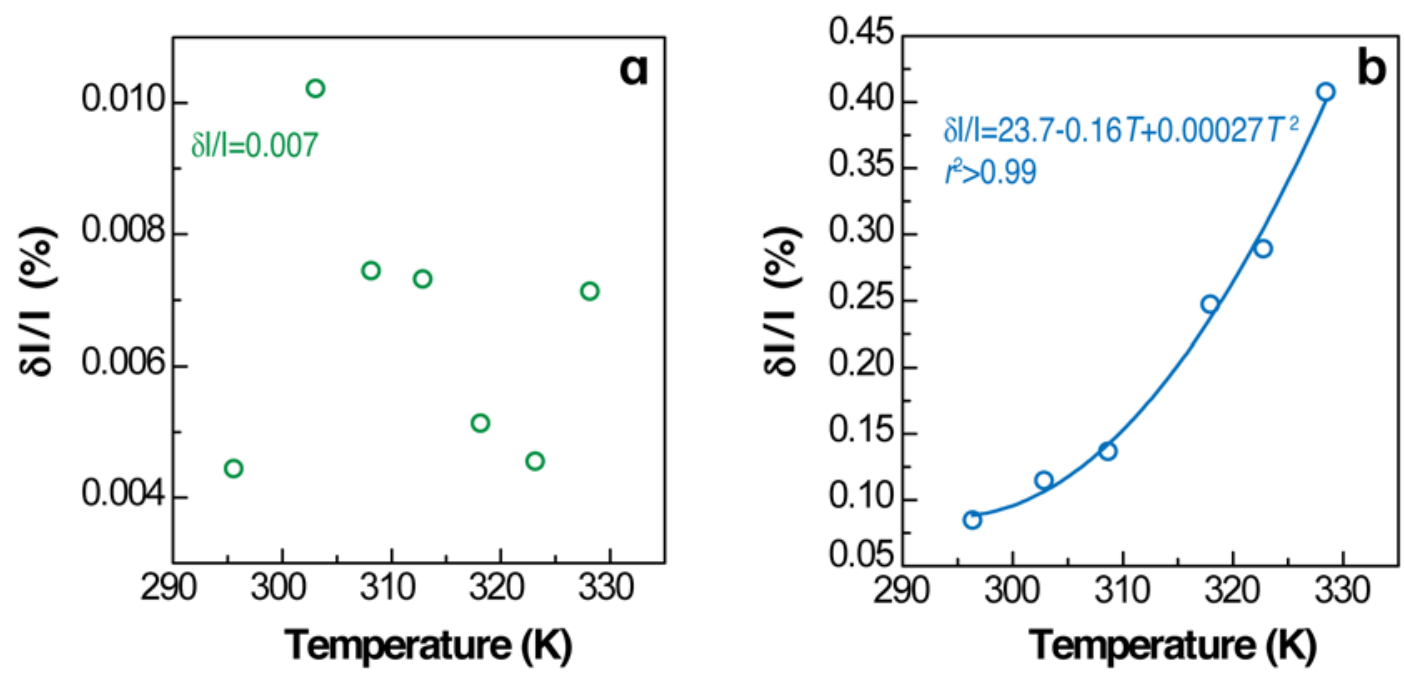

Figure S15. Signal-to-noise ratio, $\delta / / l$, in the calibration temperature range for (a) btfaand (b) DNPD-based polymeric micelles. For DNPD-based material, a polynomial function was fitted to the experimental data (presented in (b)) whereas for the btfa-based material the arithmetic average value $(0.007 \%)$ was used due to an erratic temperature dependence. 


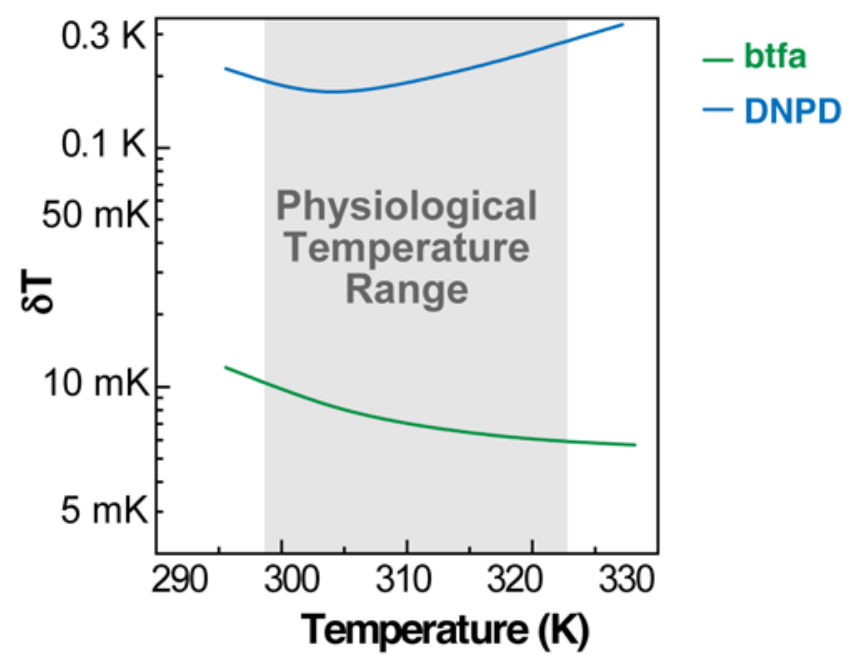

Figure S16. The temperature uncertainty, $\delta T(T)$ of btfa- and DNPD-based polymeric micelles.

\section{Cell cultures and temperature imaging}

\section{Cell cultures}

MDA-MB-468 breast cancer cell lines were cultured in commercial DMEM (Dulbecco's Modified Eagle Medium) high glucose medium without phosphate (GIBCO), supplemented with 10\% (v/v) fetal bovine serum (FBS), and $100 \mathrm{U} / \mathrm{mL}$ penicillin and $100 \mu \mathrm{g} / \mathrm{mL}$ streptomycin and kept in a thermostatic incubator (Heraeus Cell) in saturated humid air with $5 \% \mathrm{CO}_{2}$ at $37{ }^{\circ} \mathrm{C}(310 \mathrm{~K})$. Successive subcultures and media changes were done every 2 or 3 days. Cells viability was calculated by the Trypan blue (Sigma) exclusion technique. Manipulations of cell cultures were carried out inside a vertical laminar flow cabinet (Telstar) in sterility conditions.

\section{Preparation of cell samples for temperature imaging}

In total, about 470,000 cells were seeded on coverslips in 6-well plates $24 \mathrm{~h}$ before the treatments with the micelles. Then, the cells were incubated with the DNPD-based polymeric micelles for $24 \mathrm{~h}$, with different contents of the original suspension of the thermometric probes (0\%, 10\%, 20\%, and 40\%, v/v). In order to eliminate the presence of non-internalized nanoparticles, the supernatants were aspirated out and the cells were washed 3 times with cold $\mathrm{NaCl} 0.9 \%$, fixed 
with $4 \%(w / v)$ paraformaldehyde for 10 minutes, washed again three times with the same solution and mounted for microscopy. As a control, MDA-MB-468 cells were cultured in the absence of the $\mathrm{Ln}^{3+}$-bearing polymeric micelles and prepared in the same conditions.

The cell internalization ratio of the micelles was estimated by analysis of the lanthanide content in the supernatant after an incubation period of $24 \mathrm{~h}$. The obtained values were $12 \%, 14 \%$ and $10 \%$ for the original suspension volume ratios in the culture of $10 \%, 20 \%$ and $40 \%$, respectively.

\section{Temperature imaging of fixed cells}

Cell culture samples of cells incubated in the presence of the thermometric micelles were placed in a coverslip and examined in the microscope in three different imaging modes: (1) phase contrast, (2) full-color fluorescence, and (3) split fluorescent channels (for further temperature imaging). The cells cultures that have not incubated with the thermometric micelles were observed as a reference.

\section{Temperature imaging of living cells}

About 470000 cells were cultivated in $35 \mathrm{~mm}$ glass-bottom dishes (ibidi) in DMEM in $2 \mathrm{ml}$ of DMEM without phosphates with 10\% SFB and antibiotics) for $24 \mathrm{~h}$. Then, $0.2 \mathrm{~mL}$ of the thermometric micelle suspension was added and left incubating for another $24 \mathrm{~h}$ at $37^{\circ} \mathrm{C}$. Then, we removed the medium, washed twice with DMEM medium without phosphates and SFB, and then two more times with $0.9 \% \mathrm{NaCl}$. Finally, we added $2 \mathrm{~mL}$ of $0.9 \% \mathrm{NaCl}+$ glucose $0.9 \mathrm{mg} \cdot \mathrm{mL}^{-1}$ and the cell culture dishes were placed in a thermostatic holder as depicted in Figure S13. The temperature of the culture is measured with a fiber optic temperature sensor (Neoptix Reflex). The temperature imaging experiments were performed at different culture temperatures, $23,28,30$ and $30.4^{\circ} \mathrm{C}$, using an exposure time of $6 \mathrm{~s}$. 


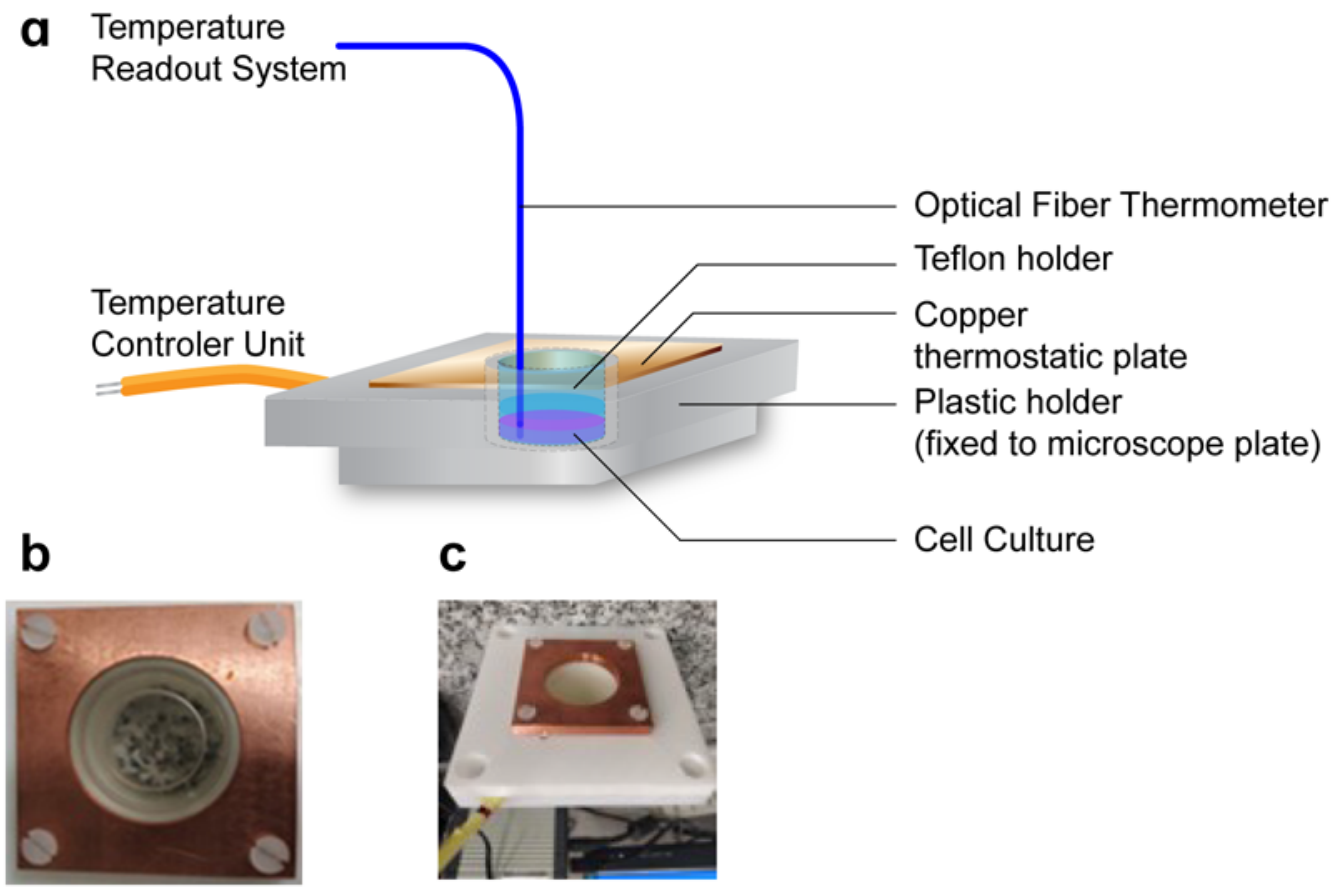

Figure S17. Thermostatic sample holder used in life cell imaging experiments. (a) General scheme of the sample holder (b) top and (c) perspective photograph of the copper thermostatic plate.
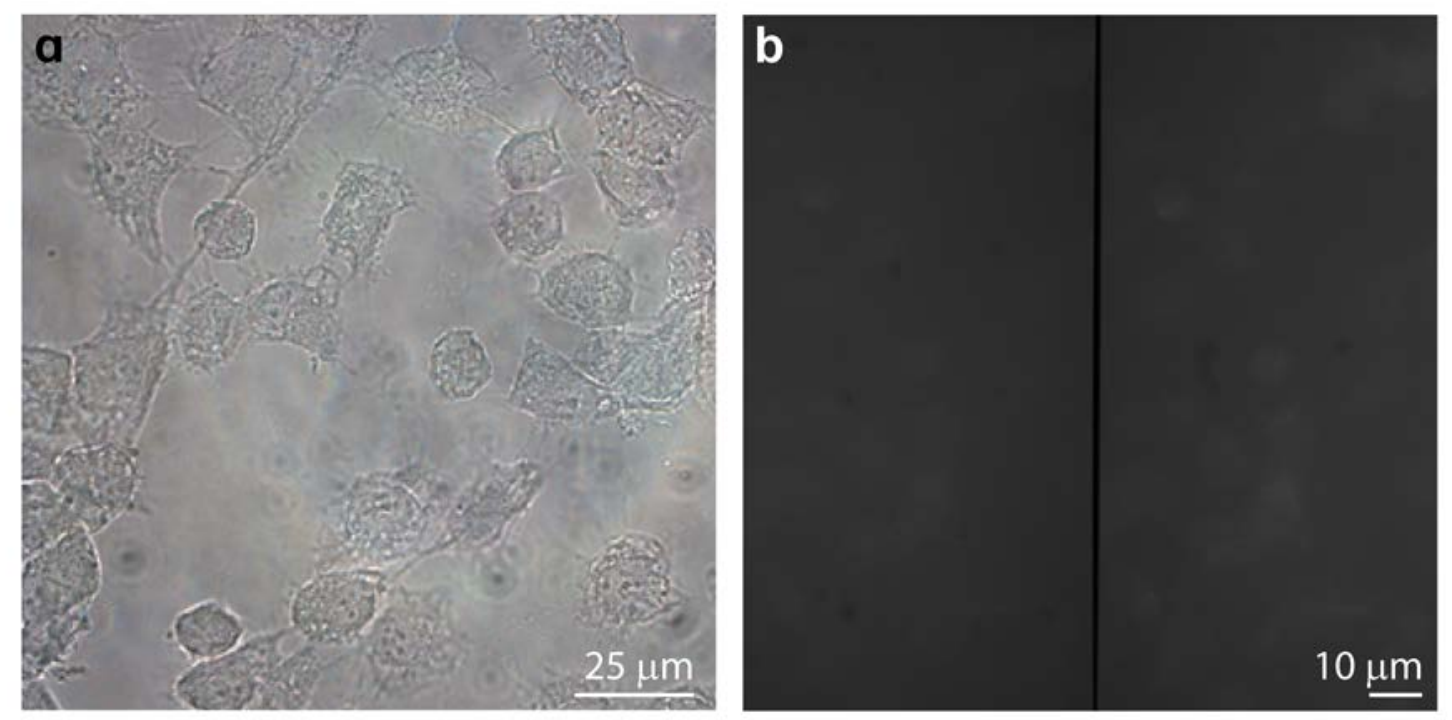

Figure S18. Control microscope images of control MDA-MB-468 cells before incubation with the micelles. (a) Phase contrast and (b) Split image using $365 \mathrm{~nm}$ illumination. 
A drop of the DNPD-based polymeric micelles suspension was deposited on a $35 \mathrm{~mm}$ glass-bottom dishes, used previously for live-cells observation. The microscope image recorded in the $\mathrm{Eu}^{3+}$ channel (Figure S19a) shows a heterogeneous distribution of micelles in which the brighter spots (signed in yellow) correspond to high particle aggregation. We observe a narrow temperature distribution (fwhm $1 \mathrm{~K}$ ), comparable to those recorded in the fixed MDA-MB-468 cells incubated with these micelles(Figure S19e).
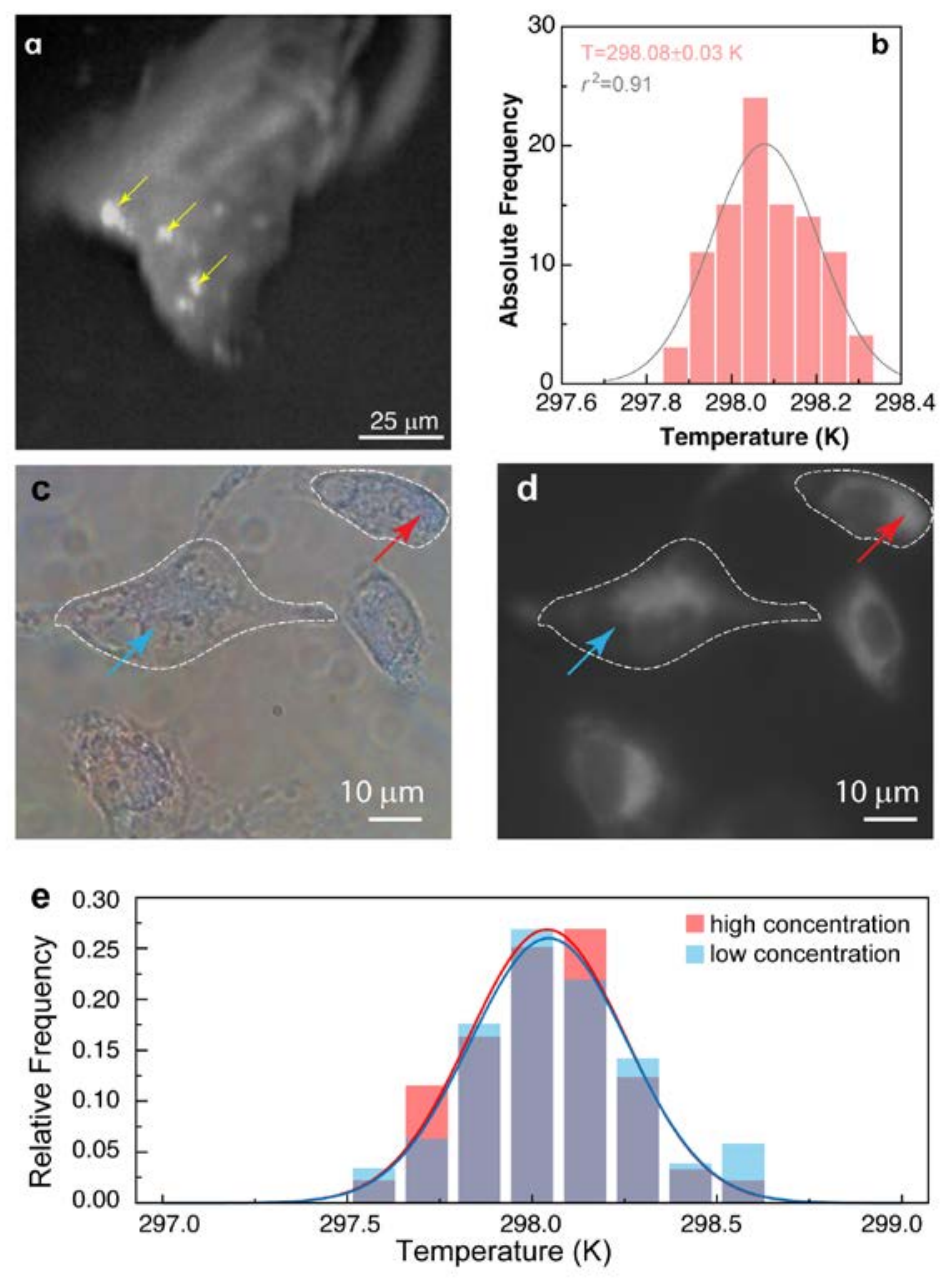

Figure S19. (a) Emission in the $\mathrm{Eu}^{3+}$ channel of DNPD-based polymeric micelles under UV-light illumination. The arrows mark the brighter spots. (b) Histogram of the temperatures measured in 100 points within the micelles. The solid line is the best fit to a Gaussian function $\left(r^{2}>0.91\right)$ with mean value \pm standard error of $298.08 \pm 0.03 \mathrm{~K}$ (the room temperature was measured as $298 \mathrm{~K}$ ). (c) Phase contrast and (d) emission images in the $\mathrm{Eu}^{3+}$ channel of fixed MDA-MB-468 cells incubated with DNPD-based polymeric micelles under UV-light illumination. The blue and red arrows mark regions of high and low concentration of the thermometric probes, as can be observed in (c). (e) Temperature histograms on the regions signed with the blue and red arrows in (c) and (d). The lines are the best fits to experimental data using Gaussian functions $\left(r^{2}>0.97\right)$ corresponding to mean temperatures of $298.04 \pm 0.04 \mathrm{~K}$ and $298.04 \pm 0.05 \mathrm{~K}$ for the blue and red lines, respectively. 

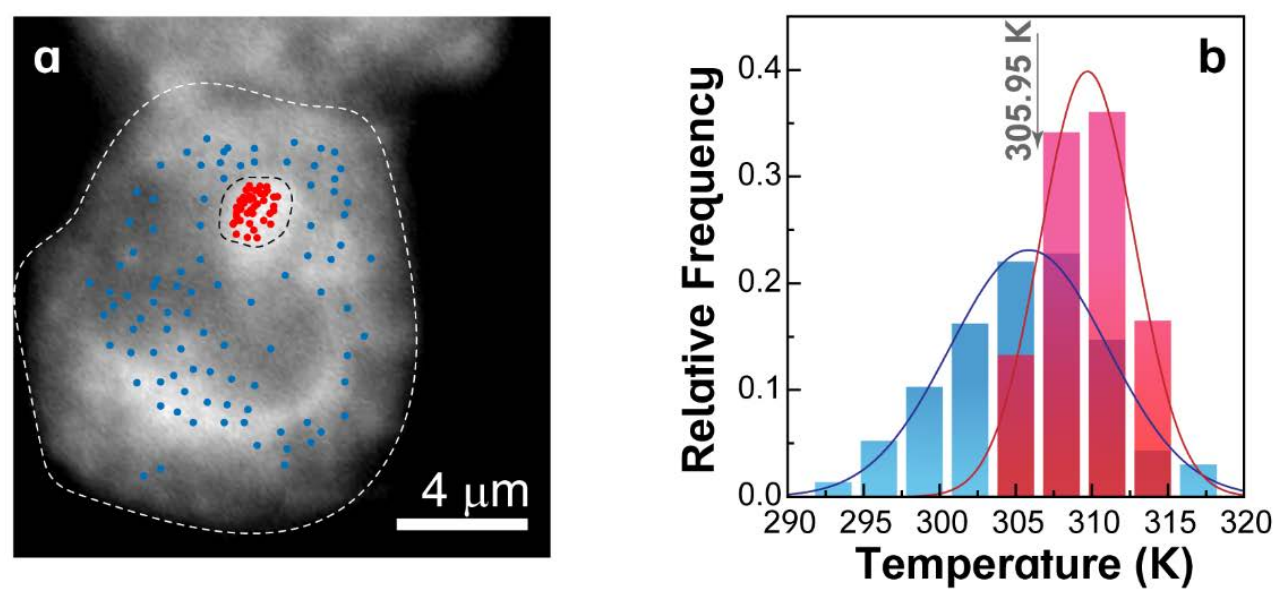

Figure S20. (a) Emission in the $\mathrm{Eu}^{3+}$ channel of MDA-MB-468 cells incubated with DNPD-based polymeric micelles recorded at $305.99 \mathrm{~K}$. The blue and red points mark, respectively, the locations used for temperature determination of the darker (100 points) and brighter (50 points) regions. (b) Temperature histograms obtained from the selected points indicated in (a). The blue and red colors correspond to the blue (dark regions) and red (bright regions) points, respectively and the solid lines represent the best fits to Gaussian functions $\left(r^{2}>0.98\right)$.

The time dependence of the intracellular temperature while the external controller is setting the temperature of the culture was recorded (Figure S21). The temperature stabilization of the cells is monitored with a time step on the order of half-a-minute, and the steady-state regime is reached at about 3 minutes after the activation of the external temperature controller.
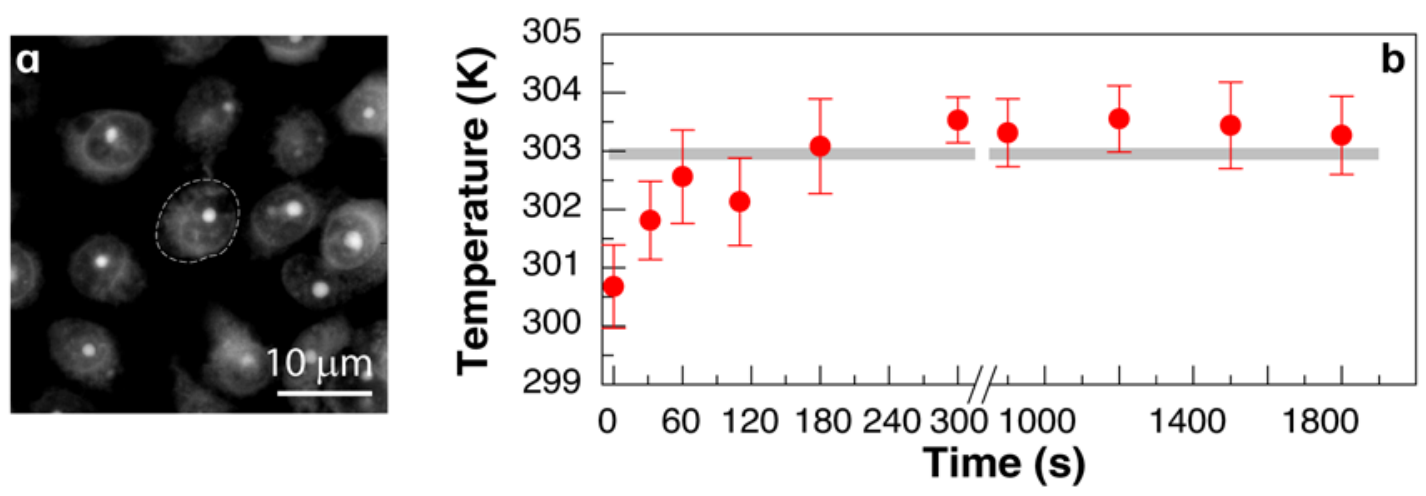

Figure S21. (a) Emission in the $\mathrm{Eu}^{3+}$ channel of MDA-MB-468 cells incubated with DNPD-based polymeric micelles under UV-light illumination $(365 \mathrm{~nm})$. The interrupted line delimits the cell whose temperature is tracked over time. (b) Temporal dependence of the mean temperature within the delimited cell. The error bars are the standard error and the horizontal bar marks the temperature set in the external temperature controller. 
Optical properties of DNPD-based polymeric micelles in cell culture medium
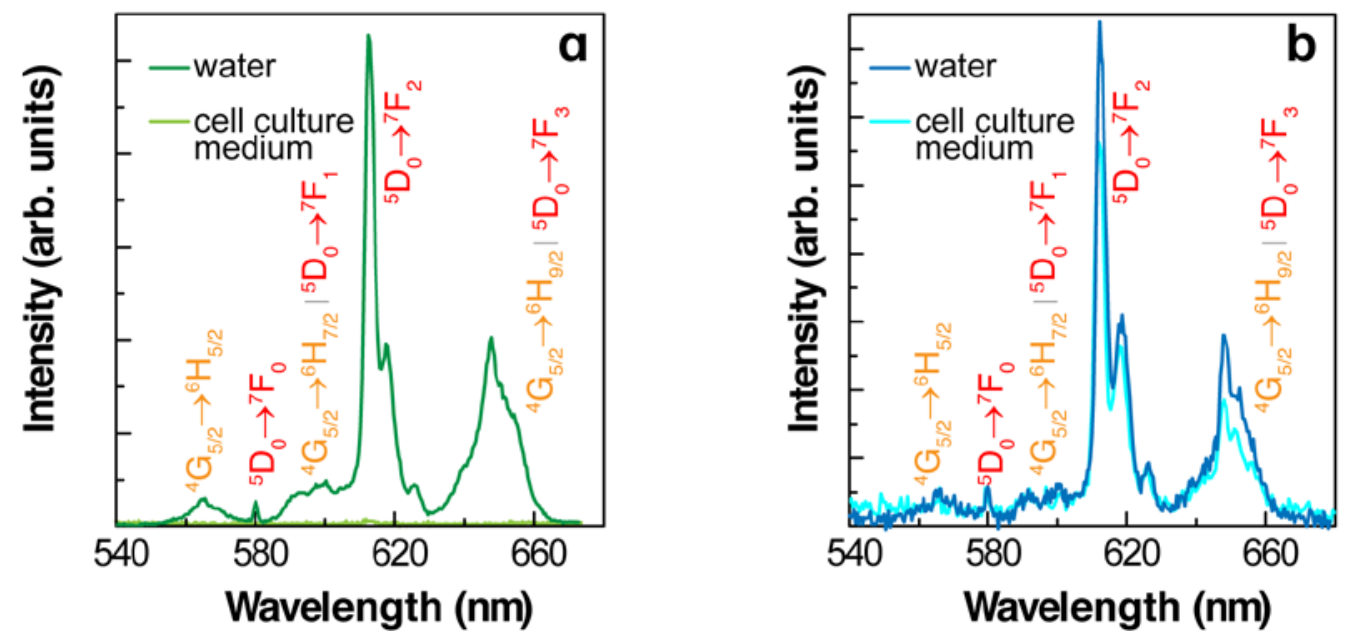

Figure S22. Room temperature emission spectra of (a) btfa- and (b) DNPD-based micelles under $355 \mathrm{~nm}$ and $405 \mathrm{~nm}$ excitation, respectively. The spectra were recorded for using water or DMEM cell culture media (incubated at $310 \mathrm{~K}$ for $24 \mathrm{~h}$ ) as solvents. 

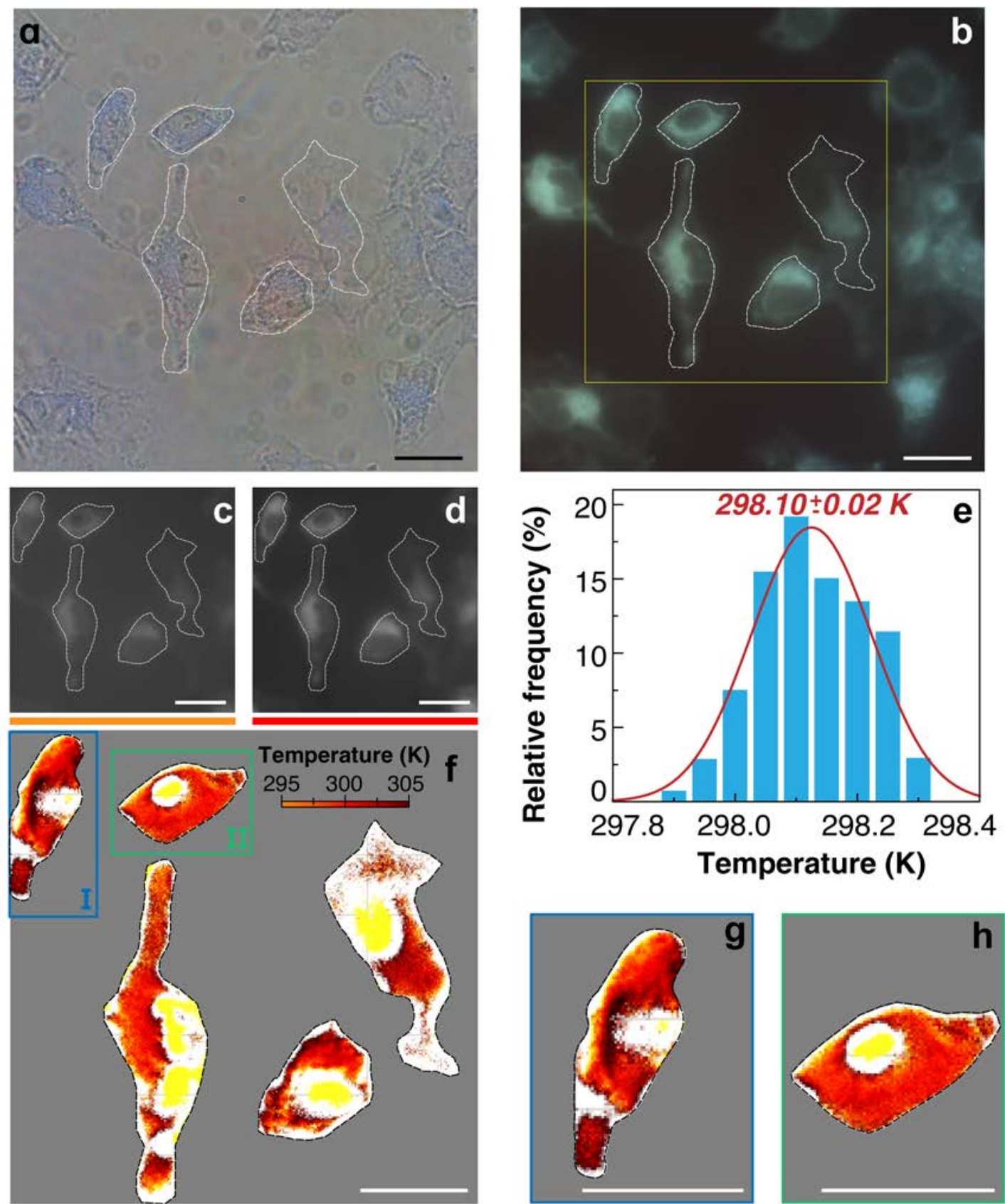

Figure S23. (a) Phase contrast and (b) fluorescence microscopy images of fixed MDAMB-468 cells at $298.0 \mathrm{~K}$. The yellow delimitated area in (b) is split in (c) $I_{1}$ and (d) $I_{2}$. (e) The histogram of the temperature distribution using 100 points in the I and II areas follows a Gaussian curve of mean value \pm standard error 298.10 $\pm 0.02 \mathrm{~K} \quad\left(r^{2}>0.95\right)$, accordingly with the cell culture temperature. The calculated temperature map of $I_{1}$ and $I_{2}$ channels is presented in (f). (g) and (h) Temperature mappings on the I and II areas, respectively. All scale bars correspond to $10 \mu \mathrm{m}$. 

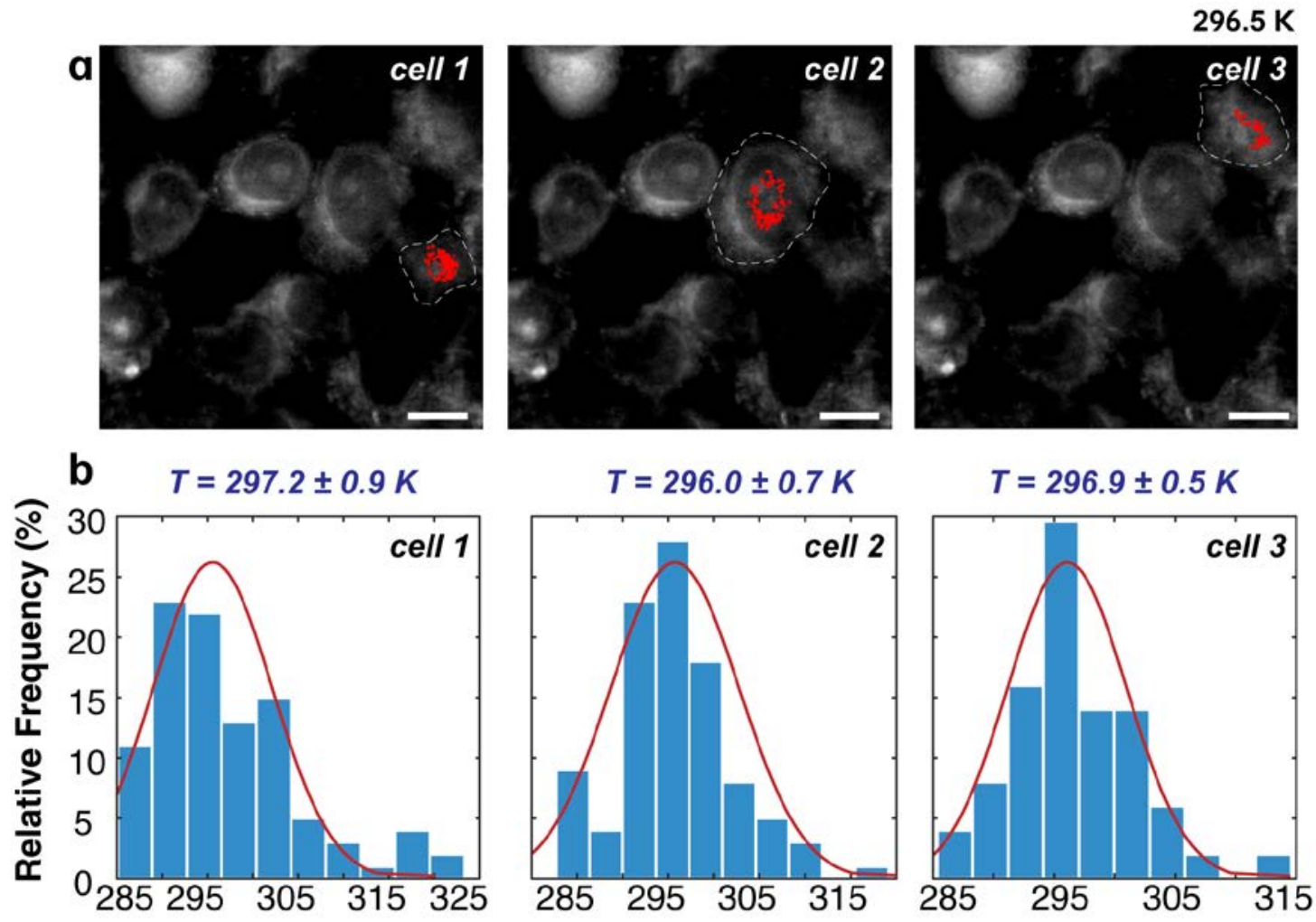

Figure S24. (a) Temperature maps of single living cells at $296.5 \mathrm{~K}$. The temperature distributions were sampled in $N>100$ points (marked in red, randomly distributed in a radius of $3.25 \mu \mathrm{m}$ around the user-selected points) for each from 3 cells at indicated temperatures. Scale bars correspond to $10 \mu \mathrm{m}$. (b) The corresponding temperature histograms constructed from the temperature distributions. The lines are the best fit to Gaussian functions $\left(r^{2}>0.89\right)$ temperature values presented correspond to the mean \pm standard error.

Table S8. List of illustrative luminescent thermometers reporting intracellular temperature determination and temperature gradients $(\Delta T)$ within living cells.

\begin{tabular}{cccccc}
\hline Thermometer & Cell line & Location & $\begin{array}{c}\text { Thermometric } \\
\text { method }\end{array}$ & $\Delta$ T (K) & Ref. \\
\hline QD655 & $\begin{array}{c}\text { HeLa, } \\
\text { NIH3T3 }\end{array}$ & $\begin{array}{c}\text { Whole cell } \\
\text { Near the } \\
\text { QD655 }\end{array}$ SH-SY5Y & $\begin{array}{c}\text { Wavelength shift } \\
\text { Intensity } \\
\text { ratio }\end{array}$ & $\begin{array}{c}\sim 15 \\
\text { Large } \\
\text { variance }\end{array}$ & 30 \\
$\begin{array}{c}\text { Polymer } \\
\text { encapsulated } \\
\text { quantum dot } \\
\text { Targeted } \\
\text { organic dye }\end{array}$ & HepG2 & Whole cell & Intensity & $\sim 15$ & 31 \\
\hline
\end{tabular}




\section{Supplementary references}

1. Belser, P.; Bernhard, S.; Guerig, U. Synthesis of Mono- and Dialkylsubstituted 1,10-Phenanthrolines. Tetrahedron 1996, 52, 2937-2944, DOI: 10.1016/0040-4020(95)01118-8.

2. Hara, K.; Sugihara, H.; Singh, L. P.; Islam, A.; Katoh, R.; Yanagida, M.; Sayama, K.; Murata, S.; Arakawa, H. New Ru(II) Phenanthroline Complex Photo Sensitizers Having Different Number of Carboxyl Groups for Dye-Sensitized Solar Cells. J. Photoch. Photobio. A 2001, 145, 117-122, DOI: 10.1016/S10106030(01)00570-6.

3. Bossmann, S. H.; Ghatlia, N. D.; Ottaviani, M. F.; Turro, C.; Durr, H.; Turro, N. J. Synthesis and Characterization of Nitroxide-Linked Ruthenium Complexes as Molecular Probes for Microheterogeneous Environments. Synthesis-Stuttgart 1996, 1313-1319, DOI: 10.1055/s-1996-4384.

4. Chandler, C. J.; Deady, L. W.; Reiss, J. A. Synthesis of Some 2,9Disubstituted-1,10-Phenanthrolines. J. Heterocyclic. Chem. 1981, 18, 599-601, DOI: $10.1002 /$ jhet.5570180332.

5. $\quad$ Piñol, R.; Brites, C. D. S.; Bustamante, R.; Martínez, A.; Silva, N. J. O.; Murillo, J. L.; Cases, R.; Carrey, J.; Estepa, C.; Sosa, C., et al. Joining TimeResolved Thermometry and Magnetic-Induced Heating in a Single Nanoparticle Unveils Intriguing Thermal Properties. ACS Nano 2015, 9, 3134-3142, DOI: 10.1021/acsnano.5b00059.

6. McGehee, M. D.; Bergstedt, T.; Zhang, C.; Saab, A. P.; O'Regan, M. B.; Bazan, G. C.; Srdanov, V. I.; Heeger, A. J. Narrow Bandwidth Luminescence from Blends with Energy Transfer from Semiconducting Conjugated Polymers to Europium Complexes. Adv. Mater. 1999, 11, 1349-1354, DOI: 10.1002/(Sici)1521-4095(199911)11:16<1349::Aid-Adma1349>3.0.Co;2-W.

7. Binnemans, K.; Lenaerts, P.; Driesen, K.; Gorller-Walrand, C. A Luminescent Tris(2-Thenoyltrifluoroacetonato) Europium(III) Complex Covalently Linked to a 1,10-Phenanthroline-Functionalised Sol-Gel Glass. J. Mater. Chem. 2004, 14, 191-195, DOI: 10.1039/B311128H

8. $\quad$ Frisch, M. J.; Trucks, G. W.; Schlegel, H. B.; Scuseria, G. E.; Robb, M. A.; Cheeseman, J. R.; Scalmani, G.; Barone, V.; Petersson, G. A.; Nakatsuji, H., et al. Gaussian 09 Rev. A.02, Gaussian, Inc., Wallingford, CT: 2016.

9. Miertus, S.; Scrocco, E.; Tomasi, J. Electrostatic Interaction of a Solute with a Continuum - a Direct Utilization of Abinitio Molecular Potentials for the Prevision of Solvent Effects. Chem. Phys. 1981, 55, 117-129, DOI: 10.1016/0301-0104(81)85090-2.

10. Miertus, S.; Tomasi, J. Approximate Evaluations of the Electrostatic FreeEnergy and Internal Energy Changes in Solution Processes. Chem. Phys. 1982, 65, 239-245, DOI: 10.1016/0301-0104(82)85072-6.

11. Pascualahuir, J. L.; Silla, E.; Tunon, I. GEPOL: An Improved Description of Molecular-Surfaces. III. A New Algorithm for the Computation of a SolventExcluding Surface. J. Comput. Chem. 1994, 15, 1127-1138, DOI: $10.1002 /$ jcc.540151009.

12. Chai, J. D.; Head-Gordon, M. Long-Range Corrected Hybrid Density Functionals with Damped Atom-Atom Dispersion Corrections. Phys. Chem. Chem. Phys. 2008, 10, 6615-6620, DOI: 10.1039/b810189b. 
13. Dolg, M.; Stoll, H.; Preuss, H. Energy-Adjusted Abinitio Pseudopotentials for the Rare-Earth Elements. J. Chem. Phys. 1989, 90, 1730-1734, DOI: 10.1063/1.456066.

14. Malta, O. L. Ligand-Rare-Earth Ion Energy Transfer in Coordination Compounds. A Theoretical Approach. J. Lumin. 1997, 71, 229-236, DOI 10.1016/S0022-2313(96)00126-3.

15. Malta, O. L.; Silva, F. R. G. E. A Theoretical Approach to Intramolecular Energy Transfer and Emission Quantum Yields in Coordination Compounds of Rare Earth Ions. Spectrochim. Acta A 1998, 54, 1593-1599, DOI: 10.1016/S1386-1425(98)00086-9.

16. Longo, R.; Silva, F. R. G. E.; Malta, O. L. A Theoretical Study of the Energy-Transfer Process in [Eu $\subset$ Bpy.Bpy.Bpy] ${ }^{3+}$ Cryptates: A Ligand-to-Metal Charge-Transfer State? Chem. Phys. Lett. 2000, 328, 67-74, DOI: 10.1016/S0009-2614(00)00898-8.

17. Malta, O. L. Mechanisms of Non-Radiative Energy Transfer Involving Lanthanide Ions Revisited. J. Non-Cryst. Solids 2008, 354, 4770-4776, DOI: 10.1016/j.jnoncrysol.2008.04.023.

18. Silva, F. R. G. E.; Malta, O. L. Calculation of the Ligand-Lanthanide Ion Energy Transfer Rate in Coordination Compounds: Contributions of Exchange Interactions. J. Alloy. Compd. 1997, 250, 427-430, DOI: 10.1016/S09258388(96)02563-7.

19. Brito, H. F.; Malta, O. M. L.; Felinto, M. C. F. C.; Teotonio, E. E. S. Luminescence Phenomena Involving Metal Enolates. In The Chemistry of Metal Enolates; Zabicky, J., Ed. Wiley-VCH Verlag GmbH: Germany, 2009; pp 131177.

20. Carnall, W. T.; Crosswhite, H.; Crosswhite, H. M. Energy Level Structure and Transition Probabilities in the Spectra of the Trivalent Lanthanides in $\mathrm{LaF}_{3}$; Argonne Nat. Lab. 78-XX-95 Rep.; Argonne Nat. Lab. 78-XX-95 Rep.: 1977.

21. Judd, B. R. Optical Absorption Intensities of Rare-Earth lons. Phys. Rev. 1962, 127, 750-761, DOI: 10.1103/PhysRev.127.750.

22. Ofelt, G. S. Intensities of Crystal Spectra of Rare-Earth Ions. J. Chem. Phys. 1962, 37, 511-520, DOI: 10.1063/1.1701366.

23. Malta, O. L. Theoretical Crystal-Field Parameters for the $\mathrm{YOCl}-\mathrm{Eu}^{3+}$ System. A Simple Overlap Model. Chem. Phys. Lett. 1982, 88, 353-356, DOI: 10.1016/0009-2614(82)87103-0.

24. Malta, O. L. A Simple Overlap Model in Lanthanide Crystal-Field Theory. Chem. Phys. Lett. 1982, 87, 27-29, DOI: 10.1016/0009-2614(82)83546-X.

25. Ofelt, G. Structure of the $f^{6}$ Configuration with Application to Rare - Earth Ions. J. Chem. Phys. 1963, 38, 2171-2180, DOI: 10.1063/1.1733947.

26. Malta, O. L.; Brito, H. F.; Menezes, J. F. S.; Silva, F.; Alves, S.; Farias, F. S.; de Andrade, A. V. M. Spectroscopic Properties of a New Light-Converting Device Eu(Thenoyltrifluoroacetonate)3 2(Dibenzyl Sulfoxide). A Theoretical Analysis Based on Structural Data Obtained from a Sparkle Model. J. Lumin. 1997, 75, 255-268, DOI: 10.1016/s0022-2313(97)00107-5.

27. Mooney, J.; Kambhampati, P. Get the Basics Right: Jacobian Conversion of Wavelength and Energy Scales for Quantitative Analysis of Emission Spectra (Vol 4, Pg 3316, 2013). J. Phys. Chem. Lett. 2014, 5, 3497-3497, DOI: 10.1021/jz502066v.

28. Brites, C. D. S.; Lima, P. P.; Silva, N. J. O.; Millán, A.; Amaral, V. S.; Palacio, F.; Carlos, L. D. Thermometry at the Nanoscale. Nanoscale 2012, 4, 4799-4829, DOI: 10.1039/c2nr30663h. 
29. Yang, J. M.; Yang, H.; Lin, L. W. Quantum Dot Nano Thermometers Reveal Heterogeneous Local Thermogenesis in Living Cells. ACS Nano 2011, 5 , 5067-5071, DOI: 10.1021/Nn201142f.

30. Tanimoto, R.; Hiraiwa, T.; Nakai, Y.; Shindo, Y.; Oka, K.; Hiroi, N.; Funahashi, A. Detection of Temperature Difference in Neuronal Cells. Sci. Rep.UK 2016, 6, 22071, DOI: 10.1038/srep22071.

31. Liu, H. L.; Fan, Y. Y.; Wang, J. H.; Song, Z. S.; Shi, H.; Han, R. C.; Sha, Y. L.; Jiang, Y. Q. Intracellular Temperature Sensing: An Ultra-Bright Luminescent Nanothermometer with Non-Sensitivity to $\mathrm{pH}$ and Ionic Strength. Sci. Rep-.UK 2015, 5, 14879, DOI: 10.1038/srep14879.

32. Chrétien, D.; Bénit, P.; Ha, H. H.; Keipert, S.; El-Khoury, R.; Chang, Y. T.; Jastroch, M.; Jacobs, H. T.; Rustin, P.; Rak, M. Mitochondria Are Physiologically Maintained at Close to 50 Degrees ${ }^{\circ} \mathrm{C}$ ? PLOS Biol. 2018, 16, e2003992, DOI: 10.1371/journal.pbio.2003992. 